\title{
MODIFIED GRADED HENNINGS INVARIANTS FROM UNROLLED QUANTUM GROUPS AND MODIFIED INTEGRAL
}

\author{
NATHAN GEER, NGOC PHU HA, AND BERTRAND PATUREAU-MIRAND
}

\begin{abstract}
The second author constructed a topological ribbon Hopf algebra from the unrolled quantum group associated with the super Lie algebra $\mathfrak{s l}(2 \mid 1)$. We generalize this fact to the context of unrolled quantum groups and construct the associated topological ribbon Hopf algebras. Then we use such an algebra, the discrete Fourier transforms, a symmetrized graded integral and a modified trace to define a modified graded Hennings invariant. Finally, we use the notion of a modified integral to extend this invariant to empty manifolds and show that it recovers the CGP-invariant.
\end{abstract}

MSC: 57M27, 17B37.

Key words: Unrolled quantum group, topological ribbon Hopf algebra, Hennings type invariant, discrete Fourier transform, modified integral.

\section{INTRODUCTION}

It is known that the category of modules over the semi-restricted quantum group at a root of unity produces 3-manifold invariants (see [6]). In [17], the second author used the semi-restricted quantum group associated to the super Lie algebra $\mathfrak{s l}(2 \mid 1)$ (not its category of modules) to define a Virelizier-Hennings type 3-manifold invariant (see [20, 35]). Here we generalize the latter construction to a large setting of Hopf algebras.

For the simplest example of a semi-restricted quantum group see Example 2.3 below. By adding Cartan elements this quantum group has been extended to a Hopf algebra called the unrolled quantum group, see for example [6, 12, 7]. In [1], Andruskiewitsch and Schweigert consider unrolled Hopf algebras which are a generalization of the unrolled quantum groups containing the case of Lie (super)algebras and more general diagonal Nichols algebra (see [19]). In this paper we propose a general construction which should produce quantum 3-manifold invariants for all of these unrolled Hopf algebras. To prove this one needs to show these algebras satisfy the five axioms listed in this paper.

Let us now summarize these axioms. Starting with a free abelian group $\Lambda$ of rank $r$ we consider three algebras $\mathcal{U}, \mathcal{U}^{H}$ and $\mathcal{U}_{\overline{0}}=\mathcal{U} / I_{\overline{0}}$ 
which are generalizations of the semi-restricted, unrolled and small quantum groups, respectively (for the simplest example of these quantum groups see Example 2.3 below). Loosely speaking, the axioms we require are:

Axiom 1: $\mathcal{U}$ is a $\Lambda$-graded pivotal Hopf algebra (graded by "weights"). Axiom 2: There exists a quasi R-matrix for $\mathcal{U}^{H}$.

Axiom 3: $\mathcal{U}_{\overline{0}}$ is unimodular.

Axiom 4: The integral of the twist and of its inverse are non zero.

Axiom 5: There exists a projective $\mathcal{U}^{H}$-module whose restriction to a $\mathcal{U}$-module remains projective.

We will prove that the semi-restricted, unrolled and small quantum groups associated to a simple Lie algebra of rank $r$ satisfy these five axioms and thus lead to the 3-manifold invariants define in this paper.

The first main result of this paper is to embed the unrolled quantum group $\mathcal{U}^{H}$ into a topological ribbon Hopf algebra $\widehat{\mathcal{U}^{H}}$ which has a topology of a complete nuclear space. In particular, we give a completion of the unrolled quantum group which is a topological ribbon Hopf algebra describe as follows. The subalgebra generated by the Cartan Lie algebra $\mathfrak{H}$ in this unrolled quantum group is embedded into the space $\mathscr{C}^{\omega}\left(\mathfrak{H}^{*}\right)$ of holomorphic functions on the dual space $\mathfrak{H}^{*}$. Then the unrolled quantum group is embedded in its completion which is a complete nuclear space and has the topology of uniform convergence on compact sets. Using this completion we show the quasitriangular structure of the small quantum group can be lifted to a topological quasitriangular structure and a topological ribbon Hopf algebra. This leads to a large class of topological ribbon Hopf algebra. In a different context, Markus J. Pflauma and Martin Schottenloher [28] already consider other kinds of nuclear Hopf algebras and their holomorphic deformations.

The techniques discussed above were first used by the second author to defined a topological ribbon Hopf algebra from the super Lie algebra $\mathfrak{s l}(2 \mid 1)$. In [17], Ha used this algebra, its associated universal invariant of links, a discrete Fourier transforms and a right $G$-integral to construct an invariant of Virelizier-Hennings type of 3-manifolds decorated by a cohomology class.

Using different techniques a modifed Hennings type invariant is given in [10]. This is an invariant defined on pairs $(M, T)$ consisting of a 3 manifold $M$ and a bichrome graph $T$ inside. The main ingredients of the construction in [10] consists of a finite dimensional Hopf algebra $H$ and a modified trace of the category $H$-mod of finite dimensional left modules over $H$. The second main result of this paper is to define a modified graded Hennings type invariant for the unrolled quantum groups satisfying the five axioms of this paper. 
The modified invariants of this paper are thus graded versions of the modified Hennings invariants of [10]. In [9] the later invariants are shown to give a (non-graded) Hennings type formula for the ReshetikhinTuraev type quantum invariants of [6] associated to zero cohomology classes. Here we give a similar modified graded Hennings-Virelizier type formula for the graded version of the invariants of [6] with nonzero cohomology classes. To do this we introduce a new algebraic tool called the modified integral.

Let us discuss the organization of the paper. In Section 2, we recall some needed properties of unrolled quantum groups and their representations. Section 3 contains the construction of a topological ribbon Hopf algebra from an unrolled quantum group. It also describes some special elements called power elements which will be used to color the mixed coupons of the bichrome graphs. In Section 5 we adapt the construction of the universal invariant associated to a ribbon Hopf algebra. In Section 6 we define both the graded Hennings invariant $\mathrm{H}$ and its modified version $\mathrm{H}^{\prime}$ which are invariants of a admissible compatible triple $(M, \Gamma, \omega)$ where $M$ is a closed 3-manifold, $\Gamma$ is a bichrome graph inside $M$ and $\omega$ is a cohomology class of $H^{1}(M \backslash \Gamma ; G)$. In Section 7, we present the notion of a modified integral which allows us to relax the admissibility condition for the modified graded Hennings invariant and provides another way to determine the invariant. Finally, in Section 8 we explain how this invariant generalize some previously defined non semi-simple invariants.

Acknowledgments. Nathan Geer was partially supported by the NSF grant DMS-1452093. This grant also supported Ngoc Phu Ha to visit Utah State University where much of the work of this paper took place.

\section{Quasi-Triangular unrolled Hopf Algebras}

In this section we give our definition of a quasi-triangular unrolled Hopf Algebra and consider its category of weight modules. Similar algebras have been considered in [1]. We discuss why the previously defined unrolled quantum groups of $[6,12,7,13]$ are examples of the algebras define in this section.

2.1. Unrolled Hopf Algebras. Fix an integer $\ell$ and a $\ell^{\text {th }}$ root of unity $\xi=\mathrm{e}^{\frac{2 i \pi}{\ell}}$. If $(G,+)$ is a abelian group and $V$ is a vector space we say $V$ is $G$-graded if there is a decomposition $V=\bigoplus_{g \in G} V_{g}$ where each $V_{g}$ is a vector space. We say an algebra $V$ is $G$-graded if it its underlying vector space is $G$-graded, $1 \in G_{0}$ and the multiplication preserves the grading: if $v \in V_{g}$ and $w \in V_{h}$ then $v w \in V_{g+h}$. If $v \in V_{g}$ we say $v$ is homogeneous.

Let $\Lambda$ be a free abelian group of rank $r$ and $W$ be a $\Lambda$-graded finite dimensional vector space over $\mathbb{C}$ with a special degree 0 element denoted $1_{W}$. If $w \in W$ is homogeneous, then we denote its degree by $|w| \in \Lambda$. 
Let $\Lambda^{*}=\operatorname{Hom}_{\mathbb{Z}}(\Lambda, \mathbb{Z})$ be the abelian group of group morphism between $\Lambda$ and $\mathbb{Z}$. Fix a basis $\left\{a_{1}, \ldots, a_{r}\right\}$ of $\Lambda^{*}$. Finally, let $\mathfrak{H}=\Lambda^{*} \otimes_{\mathbb{Z}} \mathbb{C}$.

The group ring $\mathbb{C}\left[\Lambda^{*}\right]$ is the free vector space on $\Lambda^{*}$ over $\mathbb{C}$ which is generated by the formal variables $\left\{K^{a}: a \in \Lambda^{*}\right\}$, with the relation $K^{a+b}=K^{a} K^{b}$ for any $a, b \in \Lambda^{*}$. In particular, $\mathbb{C}\left[\Lambda^{*}\right]$ can be identified with the ring of Laurent polynomials in the $r$ variables $K_{i}:=K^{a_{i}}$. It has the structure of a Hopf algebra where each element of the set $\left\{K^{a}: a \in \Lambda^{*}\right\}$ is a group-like element.

Recall that a Hopf algebra is pivotal if the square of the antipode can be expressed via the conjugation by a group-like element, called pivot $g$. We assume the following axiom.

Axiom 1. There exists a $\Lambda$-graded pivotal Hopf algebra $\mathcal{U}$ with underlying vector space

$$
\mathcal{U}=W \otimes_{\mathbb{C}} \mathbb{C}\left[\Lambda^{*}\right]
$$

which is an extension of the Hopf algebra $\mathbb{C}\left[\Lambda^{*}\right]=1_{W} \otimes \mathbb{C}\left[\Lambda^{*}\right]$ and in which for any homogenous $x \in W \cong W \otimes 1$ the following relation holds:

$$
K^{a} x K^{-a}=\xi^{a(|x|)} x
$$

for all $a \in \Lambda^{*}$.

We denote the comultiplication, counit and antipode maps of the Hopf algebra $\mathcal{U}$ by $\Delta, \epsilon$ and $S$, respectively. The axiom implies that $\mathcal{U}$ is a $\Lambda$-graded Hopf algebra in which $\mathbb{C}\left[\Lambda^{*}\right]$ is a commutative Hopf subalgebra in degree 0 . In particular, the unit is $1_{\mathcal{U}}=1_{W} \otimes 1 \in$ $W \otimes_{\mathbb{C}} \mathbb{C}\left[\Lambda^{*}\right]$. Also, for any homogenous $x, y \in \mathcal{U},|x y|=|x|+|y| \in \Lambda$, $\left|x_{(1)}\right|+\left|x_{(2)}\right|=|x|$ where $\Delta x=\sum x_{(1)} \otimes x_{(2)}$ and finally $S^{2}(x)=g x g^{-1}$.

We can now build the unrolled version $\mathcal{U}^{H}$ of $\mathcal{U}$ : Let $S \mathfrak{H}$ be the tensor symmetric algebra of $\mathfrak{H}$ which can be identified with polynomial maps on $\mathfrak{H}^{*}=\Lambda \otimes_{\mathbb{Z}} \mathbb{C}$. It has a Hopf algebra structure with elements of $\mathfrak{H}$ being primitive. Consider the semi-direct product

$$
\mathcal{U}^{H}=\mathcal{U} \rtimes S \mathfrak{H}
$$

where for each $a \in \Lambda^{*}$ the action of the associated element $H_{a} \in S \mathfrak{H}$ on a homogeneous element $x \in \mathcal{U}$ is given by $\left[H_{a}, x\right]=a(|x|) x$ or equivalently

$$
H_{a} x=x\left(H_{a}+a(|x|)\right) .
$$

Proposition 2.1. The Hopf algebra morphisms of $\mathcal{U}$ and $S \mathfrak{H}$ naturally extend (via multiplication) to comultiplication $\Delta$, counit $\epsilon$ and antipode $S$ maps on $\mathcal{U}^{H}$, making $\mathcal{U}^{H}$ into a $\Lambda$-graded pivotal Hopf algebra with the same pivot $g$ as in $\mathcal{U}$.

Proof. To see that $\mathcal{U}^{H}$ is a Hopf algebra we need to check that the extended maps satisfy the relations given in Equation (3). For example, it is easy to check that

$\Delta\left(H_{a} x\right)=\Delta\left(H_{a}\right) \Delta(x)=\Delta(x) \Delta\left(H_{a}\right)+\Delta(x) a(|x|)=\Delta\left(x\left(H_{a}+a(|x|)\right)\right)$. 
Since $g$ is grouplike $\Delta(g)=g \otimes g$ so its $\Lambda$-degree is zero. So the conjugation by $g$ is trivial on $S \mathfrak{H}$, as is the square of the antipode.

Remark 2.2. Suppose that $\Lambda^{\prime}$ is a rank $r$ sub-lattice of $\Lambda^{*}$ such that $\mathcal{U}_{\Lambda^{\prime}}=W \otimes_{\mathbb{C}} \mathbb{C}\left[\Lambda^{\prime}\right] \subset \mathcal{U}$ is a sub-Hopf algebra. Then $\mathcal{U}_{\Lambda^{\prime}}^{H}=\mathcal{U}_{\Lambda^{\prime}} \rtimes S \mathfrak{H}$ is a sub-Hopf algebra of $\mathcal{U}^{H}$. Furthermore, if the pivot $g \in \mathcal{U}$ is in $\mathcal{U}_{\Lambda^{\prime}}$ then $g$ is also a pivot for $\mathcal{U}_{\Lambda^{\prime}}$ and $\mathcal{U}_{\Lambda^{\prime}}^{H}$. We say that $\mathcal{U}_{\Lambda^{\prime}}$ and $\mathcal{U}_{\Lambda^{\prime}}^{H}$ are $\Lambda^{\prime}$ versions of $\mathcal{U}$ and $\mathcal{U}^{H}$.

We call $\mathcal{U}^{H}$ an unrolled Hopf algebra.

Example 2.3. (Continued in Examples 2.7, 4.17 and 4.22). In this example we consider the case of quantum $\mathfrak{s l}_{2}$. Assume $\ell$ is greater than 3 and let $\ell^{\prime}=\ell / \operatorname{gcd}(\ell, 2)$. Let $\mathcal{U}$ be the $\mathbb{C}$-algebra with generators $E$, $F, K$ and $K^{-1}$ with relations

$$
\begin{aligned}
& K K^{-1}=K^{-1} K=1, \quad K E K^{-1}=q E, \quad K F K^{-1}=q^{-1} F, \\
& {[E, F]=\frac{K^{2}-K^{-2}}{q-q^{-1}}, \quad E^{\ell^{\prime}}=0, \quad F^{\ell^{\prime}}=0 .}
\end{aligned}
$$

The algebra $\mathcal{U}$ is a Hopf algebra where the coproduct, counit and antipode are defined by

$$
\begin{aligned}
& \Delta(E)=1 \otimes E+E \otimes K^{2}, \quad \varepsilon(E)=0, \quad S(E)=-E K^{-2}, \\
& \Delta(F)=K^{-2} \otimes F+F \otimes 1, \quad \varepsilon(F)=0, \quad S(F)=-K^{2} F, \\
& \Delta(K)=K \otimes K \quad \varepsilon(K)=1, \quad S(K)=K^{-1} .
\end{aligned}
$$

The Hopf algebra $\mathcal{U}$ is pivotal with pivot $g=K^{2-2 \ell^{\prime}}$. The sub-Hopf algebra $\mathcal{U}_{\xi}\left(\mathfrak{s l}_{2}\right)$ generated by $E, F$ and $K^{ \pm 2}$ is known as the semirestricted quantum group where the word semi-restricted is used because $E^{\ell^{\prime}}=F^{\ell^{\prime}}=0$.

Let $\mathcal{U}^{H}$ be the $\mathbb{C}$-algebra given by generators $E, F, K^{ \pm 1}, H$ and relations in Equation (4) plus the relations:

$$
H K=K H, \quad[H, E]=E, \quad[H, F]=-F .
$$

The algebra $\mathcal{U}^{H}$ is a Hopf algebra where the coproduct, counit and antipode are defined by the above equations for $E, F$ and $K$ and

$$
\Delta(H)=H \otimes 1+1 \otimes H, \quad \varepsilon(H)=0, \quad S(H)=-H .
$$

The sub-Hopf algebra generated by $E, F, K^{ \pm 2}$ and $H$ is known as $\mathcal{U}_{\xi}^{H}\left(\mathfrak{s l}_{2}\right)$ and called the unrolled quantum group associated to $\mathfrak{s l}_{2}{ }^{1}$.

We now explain that our construction recovers these quantum groups. Let $\Lambda$ be the rank one free abelian group $\mathbb{Z}$. Let $W$ be the $\Lambda$-graded $\mathbb{C}$-vector space with basis $F^{i} E^{j}$ for $0 \leq i, j<\ell^{\prime}$ and grading given by $\left|F^{i} E^{j}\right|=j-i$. Here $\mathbb{C}\left[\Lambda^{*}\right]$ is identified with Laurent polynomials in the variable $K$, which as above is a Hopf algebra where each element

\footnotetext{
${ }^{1}$ In most of the litterature, including $[6,12,14]$, the element $K^{2}$ and $2 H$ are called $K$ and $H$ respectively.
} 
is group like. As a vector space $\mathcal{U}$ is isomorphic to $W \otimes_{\mathbb{C}} \mathbb{C}\left[\Lambda^{*}\right]$ and satisfies Axiom 1. Let $\Lambda^{\prime}=2 \Lambda^{*}$ then $\mathcal{U}_{\xi}\left(\mathfrak{s l}_{2}\right)=\mathcal{U}_{\Lambda^{\prime}}$ where $\mathcal{U}_{\Lambda^{\prime}}$ is the $\Lambda^{\prime}$ version of $\mathcal{U}$, see Remark 2.2. Moreover, $S \mathfrak{H}$ is $\mathbb{C}[H]$ and the Hopf algebra of Proposition 2.1 is isomorphic to $\mathcal{U}^{H}$ where $\mathcal{U}_{\xi}^{H}\left(\mathfrak{s l}_{2}\right)=\mathcal{U}_{\Lambda^{\prime}}^{H}$ is its $\Lambda^{\prime}$ version.

More generally, we have:

Example 2.4. (Continued in Examples 2.8, 4.18 and 4.22). Here let $\ell$ be odd and greater than 3 . Let $\mathfrak{g}$ be a simple finite dimensional complex Lie algebra of rank $r$ with a set of simple roots $\left\{\alpha_{1}, \ldots, \alpha_{r}\right\}$. Let $A=\left(a_{i j}\right)_{1 \leq i, j \leq r}$ be the Cartan matrix corresponding to these simple roots. Consider the unrolled quantum group $\mathcal{U}_{\xi}^{H}(\mathfrak{g})$ associated to $\mathfrak{g}$, given in [12]. This algebra has generated denoted $K_{\beta}, X_{i}, X_{-i}$ and $H_{\alpha_{i}}$ where $\beta$ is in the root lattice $\Lambda$ and $1 \leq i \leq r$ (for the relations see [12]). The semi-restricted quantum group $\mathcal{U}_{\xi}(\mathfrak{g})$ is the subalgebra of $\mathcal{U}_{\xi}^{H}(\mathfrak{g})$ generated by $K_{\beta}, X_{ \pm i}$ for all $\beta$ and $i$.

To describe this example we will need the following notation here and later. There exists a diagonal matrix $D=\operatorname{diag}\left(d_{1}, \ldots, d_{r}\right)$ such that $D A$ is symmetric and positive-definite $\left(D\right.$ is unique if $\left.1 \in\left\{d_{i}\right\}_{i} \subset \mathbb{Z}\right)$. Let $\Lambda$ be the root lattice which is the $\mathbb{Z}$-lattice generated by the simple roots $\left\{\alpha_{i}\right\}$ and let $\left\{\alpha_{i}^{*}\right\}$ be the dual basis of $\Lambda^{*}$. Let $\mathfrak{H}=\Lambda^{*} \otimes_{\mathbb{Z}} \mathbb{C}$ be the Cartan subalgebra of $\mathfrak{g}$ and $\mathrm{B}: \mathfrak{H}^{*} \times \mathfrak{H}^{*} \rightarrow \mathbb{C}$ be the symmetric bilinear form defined by $\mathrm{B}\left(\alpha_{i}, \alpha_{j}\right)=d_{i} a_{i j}$. For $\lambda \in \Lambda$ let $B_{\lambda}=\mathrm{B}(\lambda, \cdot) \in \mathbb{C}\left[\Lambda^{*}\right]$ and $\Lambda^{\prime}=\left\{B_{\lambda}: \lambda \in \Lambda\right\}$. Define $H_{\alpha_{i}}=\sum_{j} a_{i j} H_{\alpha_{j}^{*}}$ so that the lattice $\Lambda^{\prime}$ also identify with the free group generated by the elements $\left\{d_{i} H_{\alpha_{i}}, i=\right.$ $1, \ldots, r\}$ in $\mathfrak{H}$.

To describe $W$, let $\beta_{1}, \ldots, \beta_{N} \in \Lambda$ be an ordering of the set of positive roots where $N=\frac{\operatorname{dim}(\mathfrak{g})-r}{2}$. For each $i=1, \ldots, N$, let $X_{ \pm \beta_{i}}$ be the positive (resp. negative) root vector of $\mathcal{U}_{\xi}(\mathfrak{g})$ (see for example [5, Section 8.1 and 9.1]). Let $W$ be the $\Lambda$-graded $\mathbb{C}$-vector space with homogeneous basis

$$
X_{\beta_{1}}^{i_{1}} X_{\beta_{2}}^{i_{2}} \ldots X_{\beta_{N}}^{i_{N}} X_{-\beta_{1}}^{j_{1}} X_{-\beta_{2}}^{j_{2}} \ldots X_{-\beta_{N}}^{j_{N}}
$$

with grading $\sum_{k=1}^{r}\left(i_{k}-j_{k}\right) \beta_{k}$ for $i_{1}, \ldots, i_{N}, j_{1}, \ldots, j_{N} \in\{0, \ldots, \ell-1\}$.

The vector space $\mathcal{U}_{\xi}(\mathfrak{g}) \simeq W \otimes_{\mathbb{C}} \mathbb{C}\left[\Lambda^{\prime}\right]$ embeds into $\mathcal{U}=W \otimes_{\mathbb{C}} \mathbb{C}\left[\Lambda^{*}\right]$. The Hopf algebra structure of $\mathcal{U}_{\xi}(\mathfrak{g})$ extends uniquely to a Hopf algebra structure on $\mathcal{U}$ such that relations (1) hold where $\left|X_{ \pm i}\right|= \pm \alpha_{i}$. Then $\mathcal{U}$ satisfies Axiom 1 with pivot given by $g=B_{2(1-\ell) \rho}$ where $\rho$ is the half sum of all positive roots (see [12]). Finally, $\mathcal{U}_{\Lambda^{\prime}}=\mathcal{U}_{\xi}(\mathfrak{g})$ and $\mathcal{U}_{\Lambda^{\prime}}^{H}=\mathcal{U}_{\xi}^{H}(\mathfrak{g})$ are the $\Lambda^{\prime}$ version of the quantum group $\mathcal{U}$ and $\mathcal{U}^{H}$.

2.2. Category of weight modules. In the following, we will use the notation $\xi^{x}:=\exp \left(\frac{2 i \pi x}{\ell}\right)=\sum_{n=0}^{\infty} \frac{1}{n !}\left(\frac{2 i \pi x}{\ell}\right)^{n}$ for $x$ a complex number or an element of a topological algebra. 
A finite dimensional $\mathcal{U}^{H}$-module $V$ is a weight module if it is a semisimple module over the subalgebra $S \mathfrak{H}$ and

$$
K^{a}=\xi^{H_{a}}
$$

as operators on $V$, for any $a \in \Lambda^{*}$. Let $\mathscr{C}^{H}$ be the tensor category of $\mathcal{U}^{H}$-weight modules. The eigenspaces for the action of $S \mathfrak{H}$ on a weight module $V$ are called weight spaces of $V$ and this action gives a $\mathfrak{H}^{*}$-grading on $V$.

Recall that a pivotal category is a tensor category with left duality $\left\{\overleftarrow{c o e v}_{V}, \overleftarrow{\mathrm{ev}}_{V}\right\}_{V}$ and right duality $\left\{\overrightarrow{\mathrm{coe}}_{V}, \overrightarrow{\mathrm{ev}}_{V}\right\}_{V}$ which satisfy certain compatibility conditions, see for example [2]. The category $\mathscr{C}^{H}$ is a pivotal category with duality maps:

$$
\begin{gathered}
\overleftarrow{\operatorname{coev}}_{V}: \mathbb{C} \rightarrow V \otimes V^{*}, \text { given by } 1 \mapsto \sum v_{i} \otimes v_{i}^{*}, \\
\overleftarrow{\mathrm{ev}}_{V}: V^{*} \otimes V \rightarrow \mathbb{C} \text {, given by } f \otimes w \mapsto f(w), \\
\overrightarrow{\operatorname{coev}}_{V}: \mathbb{C} \rightarrow V^{*} \otimes V, \text { given by } 1 \mapsto \sum v_{i}^{*} \otimes g^{-1} v_{i}, \\
\overrightarrow{\mathrm{ev}}_{V}: V \otimes V^{*} \rightarrow \mathbb{C}, \text { given by } w \otimes f \mapsto f(g w)
\end{gathered}
$$

where $\left\{v_{i}\right\}$ is a basis of $V$ and $\left\{v_{i}^{*}\right\}$ is its dual basis of $V^{*}=\operatorname{Hom}_{\mathbb{C}}(V, \mathbb{C})$.

Let

$$
G=\mathfrak{H}^{*} / \Lambda \simeq(\mathbb{C} / \mathbb{Z})^{r}
$$

then $\mathscr{C}^{H}$ is $G$-graded: a module of $\mathscr{C}^{H}$ is homogeneous of degree $\bar{\alpha} \in G$ if all its weights belong to $\bar{\alpha}$. We call $\mathscr{C}_{\bar{\alpha}}^{H}$ the full subcategory of degree $\bar{\alpha}$ homogeneous modules. One easily check that any module of $\mathscr{C}^{H}$ is a direct sum of homogeneous module and that the Hom set of two homogeneous module of different degrees is zero. We summarize this by writing

$$
\mathscr{C}^{H}=\bigoplus_{\bar{\alpha} \in G} \mathscr{C}_{\bar{\alpha}}^{H}
$$

Given an element

$$
\mathrm{Q}=\sum_{i} c_{i} \otimes c_{i}^{\prime} \in \Lambda^{*} \otimes_{\mathbb{Z}} \Lambda^{*} \subset \mathfrak{H} \otimes \mathfrak{H} \subset \mathcal{U}^{H} \otimes \mathcal{U}^{H}
$$

we define the following four maps. First, let $B: \mathfrak{H}^{*} \times \mathfrak{H}^{*} \rightarrow \mathbb{C}$ be the symmetric bilinear form given by the element $\mathbf{Q}$, in particular, $\mathrm{B}(\lambda, \mu)=\mathrm{Q}(\lambda \otimes \mu) \in \mathbb{Z}$ for $(\lambda, \mu) \in \Lambda^{2}$. Second, if $V, W$ are $\mathfrak{H}^{*}$-graded vector space then let $\mathcal{H}_{V, W}=\xi^{\mathrm{Q}}: V \otimes W \rightarrow V \otimes W$ be the operator defined by $\mathcal{H}_{V, W}(v \otimes w)=\xi^{\mathrm{B}(|v|,|w|)} v \otimes w$ for homogeneous vectors $v$ and $w$. Third, consider the map $B: \Lambda \rightarrow \Lambda^{*} \subset \mathbb{C}\left[\Lambda^{*}\right], \lambda \mapsto B_{\lambda}$ where $B_{\lambda}=\mathrm{B}(\lambda, \cdot)$. Then we have $B_{\lambda} B_{\mu}=B_{\lambda+\mu}$ in $\mathbb{C}\left[\Lambda^{*}\right]$ for $\lambda, \mu \in \Lambda$. Using this map we can define an outer automorphism $\widetilde{\mathcal{H}}$ of $\mathcal{U}^{H} \otimes \mathcal{U}^{H}$ given by

$$
\widetilde{\mathcal{H}}(x \otimes y)=x B_{|y|} \otimes B_{|x|} y .
$$


for $x, y \in \mathcal{U}^{H}$. This outer automorphism is compatible with conjugation by $\mathcal{H}$ in $\mathscr{C}^{H}$ : if $\rho_{V_{i}}: \mathcal{U}^{H} \rightarrow \operatorname{End}_{\mathscr{C}^{H}}\left(V_{i}\right)$, for $i=1,2$, are objects in $\mathscr{C}^{H}$ then

$$
\left(\rho_{V_{1}} \otimes \rho_{V_{2}}\right)(\widetilde{\mathcal{H}}(x \otimes y))=\mathcal{H}_{V_{1}, V_{2}}\left(\rho_{V_{1}}(x) \otimes \rho_{V_{2}}(y)\right) \mathcal{H}_{V_{1}, V_{2}}^{-1}
$$

for $x, y \in \mathcal{U}^{H}$.

Throughout this paper, for two spaces $X$ and $Y$ we denote their flip map as $\tau: X \otimes Y \rightarrow Y \otimes X$ which given by $x \otimes y \mapsto y \otimes x$. We call an element

$$
\check{\mathcal{R}}=\sum_{i} x_{i} \otimes y_{i} \in \mathcal{U} \otimes \mathcal{U}
$$

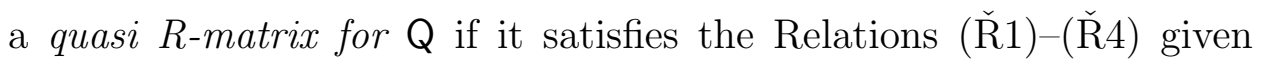
below:

$$
\begin{aligned}
\tau(\Delta u) & =\widetilde{\mathcal{H}}\left(\check{\mathcal{R}}(\Delta u) \check{\mathcal{R}}^{-1}\right), \text { for all } u \in \mathcal{U}^{H}, \\
\Delta_{1} \check{\mathcal{R}} & =\widetilde{\mathcal{H}}_{23}^{-1}\left(\check{\mathcal{R}}_{13}\right) \check{\mathcal{R}}_{23}, \\
\Delta_{2} \check{\mathcal{R}} & =\widetilde{\mathcal{H}}_{12}^{-1}\left(\check{\mathcal{R}}_{13}\right) \check{\mathcal{R}}_{12},
\end{aligned}
$$

where $\check{R}_{23}=\sum_{j} 1_{\mathcal{U}^{H}} \otimes x_{j} \otimes y_{j}, \check{R}_{12}=\sum_{j} x_{j} \otimes y_{j} \otimes 1_{\mathcal{U}^{H}}, \widetilde{\mathcal{H}}_{23}^{-1}\left(\check{\mathcal{R}}_{13}\right)=$ $\sum_{j} x_{j} \otimes\left(B_{\left|y_{j}\right|}\right)^{-1} \otimes y_{j}$ and $\widetilde{\mathcal{H}}_{12}^{-1}\left(\check{\mathcal{R}}_{13}\right)=\sum_{j} x_{j} \otimes\left(B_{\left|x_{j}\right|}\right)^{-1} \otimes y_{j}$. Finally, we require $\check{R}$ is compatible with the pivot $g$ by assuming

$$
\sum_{i} y_{i} g x_{i} B_{\left|x_{i}\right|}=\sum_{i} x_{i} g^{-1} y_{i}\left(B_{\left|x_{i}\right|}\right)^{-1} .
$$

Note, above we assume the expression of $\check{R}=\sum_{j} x_{j} \otimes y_{j}$ is given with homogeneous elements $x_{i}, y_{i} \in \mathcal{U}$.

Axiom 2. Assume Axiom 1 and $\mathcal{U}^{H}$ has an element $\check{\mathcal{R}}$ which is a quasi $R$-matrix for $\mathrm{Q}$.

In the rest of the paper, we assume Axiom 2 is true. Then Hopf algebra $\mathcal{U}^{H}$ is not quasi-triangular as $\mathcal{H} \notin \mathcal{U}^{H} \otimes \mathcal{U}^{H}$ but as we will see next the category $\mathscr{C}^{H}$ is still braided even ribbon.

Recall a braiding on a tensor category $\mathscr{C}$ consists of a family of natural isomorphisms $\left\{c_{V, W}: V \otimes W \rightarrow W \otimes V\right\}$ satisfying the Hexagon Axiom:

$c_{U, V \otimes W}=\left(\operatorname{Id}_{V} \otimes c_{U, W}\right) \circ\left(c_{U, V} \otimes \operatorname{Id}_{W}\right) \quad c_{U \otimes V, W}=\left(c_{U, W} \otimes \operatorname{Id}_{V}\right) \circ\left(\operatorname{Id}_{U} \otimes c_{V, W}\right)$ for all $U, V, W \in \mathscr{C}$. We say $\mathscr{C}$ is braided if it has a braiding. If $\mathscr{C}$ is pivotal and braided, one can define a family of natural automorphisms

$$
\theta_{V}=\left(\mathrm{Id} \otimes \overrightarrow{\mathrm{ev}}_{V}\right)\left(c_{V, V} \otimes \mathrm{Id}\right)\left(v \otimes \overleftarrow{\mathrm{coev}}_{V}\right): V \rightarrow V
$$

Following [13], we say that $\mathscr{C}$ is ribbon and the morphism $\theta$ is a twist if

$$
\theta_{V^{*}}=\left(\theta_{V}\right)^{*}
$$


for all $V \in \mathscr{C}$.

Proposition 2.5. Category $\mathscr{C}^{H}$ is ribbon with pivotal structure given above and braiding given by $c_{V, W}=\tau \circ \mathcal{H} \circ \check{\mathcal{R}}: V \otimes W \rightarrow W \otimes V$.

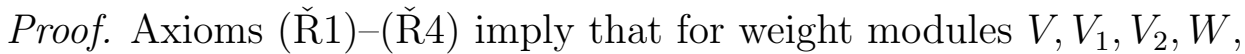

$(\check{\mathrm{R}} 1) \Longrightarrow c_{V, W}: V \otimes W \rightarrow W \otimes V$ is a morphism in $\mathscr{C}^{H}$,

$(\check{\mathrm{R}} 2) \Longrightarrow c_{V_{1} \otimes V_{2}, W}=\left(c_{V_{1}, W} \otimes \mathrm{Id}\right)\left(\mathrm{Id} \otimes c_{V_{2}, W}\right)$,

$(\check{\mathrm{R}} 3) \Longrightarrow c_{W, V_{1} \otimes V_{2}}=\left(\mathrm{Id} \otimes c_{W, V_{2}}\right)\left(c_{W, V_{1}} \otimes \mathrm{Id}\right)$,

$(\check{\mathrm{R}} 4) \Longrightarrow$ the pivotal structure and the braiding are compatible i.e. $\theta_{V}:=\left(\mathrm{Id} \otimes \overrightarrow{\mathrm{ev}}_{V}\right)\left(c_{V, V} \otimes \mathrm{Id}\right)\left(\mathrm{Id} \otimes \overleftarrow{\mathrm{coev}}_{V}\right)$ is a twist in $\mathscr{C}^{H}$

We justify with more details this last sentence. The pivotal structure gives natural isomorphisms $V^{* *} \cong V$. Using them, the dual and equivalent equality to (10) is given by

$$
\theta_{V}^{\prime}=\theta_{V} \text { where } \theta_{V}^{\prime}=\left(\overleftarrow{\mathrm{ev}}_{V} \otimes \mathrm{Id}\right)\left(\mathrm{Id} \otimes c_{V, V}\right)\left(\overrightarrow{\operatorname{coev}}_{V} \otimes \mathrm{Id}\right)
$$

Recall that $\overrightarrow{\mathrm{ev}}_{V}=\overleftarrow{\mathrm{ev}}_{V} \circ \tau \circ(g \otimes 1)$ and $\overrightarrow{\mathrm{coev}}_{V}=\left(\mathrm{Id} \otimes g^{-1}\right) \circ \tau \circ \overleftarrow{\operatorname{coev}}_{V}$ As $|g|=0$, the pivot commute with $\mathfrak{H}$. Similarly, as $\widetilde{\mathcal{H}}$ and $\tau$ are trivial on $\Delta(\mathfrak{H})$, then Relation $(\check{\mathrm{R}} 1)$ implies that $\check{R}$ commute with $\Delta(\mathfrak{H})$ and thus $\left|x_{i}\right|+\left|y_{i}\right|=0$. Then we can compute for a vector $v \in V$ with weight $\lambda$ :

$$
\begin{aligned}
\theta_{V}(v) & =\left(\mathrm{Id} \otimes \overrightarrow{\mathrm{ev}}_{V}\right)\left(c_{V, V} \otimes \mathrm{Id}\right)\left(v \otimes \overleftarrow{\mathrm{coev}}_{V}\right) \\
& =\left(\mathrm{Id} \otimes \overleftarrow{\mathrm{ev}}_{V} \tau\right)(\mathrm{Id} \otimes \rho(g) \otimes \mathrm{Id})\left(\tau \mathcal{H}_{V, V} \rho^{\otimes 2}(\check{R}) \otimes \mathrm{Id}\right)\left(v \otimes \overleftarrow{\mathrm{coe}}_{V}\right) \\
& =\sum_{i}\left(\mathrm{Id} \otimes \overleftarrow{\mathrm{ev}}_{V} \tau\right)\left(\tau \mathcal{H}_{V, V} \otimes \mathrm{Id}\right)\left(\rho\left(g x_{i}\right)(v) \otimes\left(\rho\left(y_{i}\right) \otimes \mathrm{Id}\right)(\overleftarrow{\mathrm{coev}})\right) \\
& =\sum_{i} \xi^{\mathrm{B}\left(\left|x_{i}\right|+\lambda, \lambda\right)}\left(\mathrm{Id} \otimes \overleftarrow{\mathrm{ev}}_{V} \tau\right)(\tau \otimes \mathrm{Id})\left(\rho\left(g x_{i}\right)(v) \otimes\left(\rho\left(y_{i}\right) \otimes \mathrm{Id}\right)(\overleftarrow{\mathrm{coe}})\right) \\
& =\sum_{i} \xi^{\mathrm{B}\left(\left|x_{i}\right|+\lambda, \lambda\right)}\left(\rho\left(y_{i}\right) \otimes \overleftarrow{\mathrm{ev}}_{V}\right)(\mathrm{Id} \otimes \tau)(\tau \otimes \mathrm{Id})\left(\rho\left(g x_{i}\right)(v) \otimes \operatorname{coev}\right) \\
& =\sum_{i} \xi^{\mathrm{B}\left(\left|x_{i}\right|+\lambda, \lambda\right)}\left(\rho\left(y_{i}\right) \otimes \overleftarrow{\mathrm{ev}}_{V}\right)\left(\overleftarrow{\operatorname{coev}} \otimes \rho\left(g x_{i}\right)(v)\right) \\
& =\xi^{\mathrm{B}(\lambda, \lambda)} \sum_{i} \xi^{\mathrm{B}\left(\left|x_{i}\right|, \lambda\right)} \rho\left(y_{i} g x_{i}\right)(v) .
\end{aligned}
$$

Here the third equality is because $\rho(g)$ commutes with $\mathcal{H}_{V, V}$, the fourth is because $\theta_{V}$ is $\mathfrak{H}$-equivariant so $\theta_{V}(v)$ has weight $\lambda$. This implies that the operator $\mathcal{H}_{V, V}$ is applied on vectors of weight $\lambda$ on the right (and $\left|g x_{i} v\right|$ on the left). Finally the last equality is the zig-zag relation. Similarly, we compute

$$
\theta_{V}^{\prime}(v)=\sum_{i} \xi^{\mathrm{B}\left(\lambda,\left|y_{i}\right|+\lambda\right)} \rho\left(x_{i} g^{-1} y_{i}\right)(v)=\xi^{\mathrm{B}(\lambda, \lambda)} \sum_{i} \xi^{-\mathrm{B}\left(\left|x_{i}\right|, \lambda\right)} \rho\left(x_{i} g^{-1} y_{i}\right)(v)
$$


and the equality $\theta_{V}(v)=\theta_{V}^{\prime}(v)$ follows from Relation (̌̌ 4$)$ with $B_{\left|x_{i}\right|} v=$ $\xi^{\mathrm{B}\left(\left|x_{i}\right|, \lambda\right)} v$.

Remark 2.6. Suppose that $\Lambda^{\prime}$ is a rank $r$ sub-lattice of $\Lambda^{*}$ as in Remark 2.2. Then one easily check that the analogous category of $\mathcal{U}_{\Lambda^{\prime}}^{H}$-weight module is indentified with $\mathscr{C}^{H}$. Indeed the restriction functor gives any $\mathcal{U}^{H}$-weight module a structure of $\mathcal{U}_{\Lambda^{\prime}}^{H}$-weight module but reciprocally, Condition (5) gives a unique way to extend any $\mathcal{U}_{\Lambda^{\prime}}^{H}$-weight module to a $\mathcal{U}^{H}$-module.

Notation: For $q \in \mathbb{C} \backslash\{1\}$ and $j \in \mathbb{N}$, we use

$$
[j ; q]=\frac{1-q^{j}}{1-q} \text { and }[j ; q] !=[j ; q] \cdots[1 ; q] .
$$

Example 2.7. This example builds upon Example 2.3 and is continued in Examples 4.17 and 4.22. Recall $\ell \geq 3, \ell^{\prime}=\ell / \operatorname{gcd}(\ell, 2)$ and $\mathcal{U}$ is a degree 2 extension of the semi-restricted quantum group $\mathcal{U}_{\Lambda^{\prime}}=\mathcal{U}_{\xi}\left(\mathfrak{s l}_{2}\right)$. Then a quasi R-matrix for $\mathrm{Q}=2 H \otimes H$ is given by

$$
\check{\mathcal{R}}=\sum_{j=0}^{\ell^{\prime}-1} \frac{\left(\xi-\xi^{-1}\right)^{j}}{\left[j ; \xi^{-2}\right] !} E^{j} \otimes F^{j} \in \mathcal{U} \otimes \mathcal{U}
$$

and if $v \otimes w \in V \otimes W \in \mathscr{C}^{H}$ where $v, w$ have weights $|v|$ and $|w|$, then $\mathcal{H}_{V, W}(v \otimes w)=\xi^{2|v| \cdot|w|} v \otimes w$. With slight abuse of notation we write the equality of operator on $\mathscr{C}^{H}$ as

$$
\mathcal{H}=\xi^{2 H \otimes H} .
$$

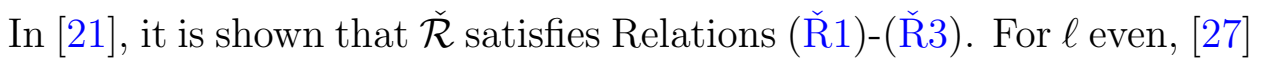
has shown an equivalent form of Relation (R 4$)$. For $\ell$ odd the proof of Example 2.8 can be applied. Hence for any $\ell \geq 3$, the quantum group $\mathcal{U}$ associated to $\mathfrak{s l}_{2}$ of Example 2.3 satisfies Axiom 2 .

Example 2.8. Here we use the notation and build upon Example 2.4 (continued in Examples 4.18 and 4.22). Recall $\mathcal{U}$ is an extension of the semi-restricted quantum group $\mathcal{U}_{\xi}(\mathfrak{g})$ associated to a simple finite dimensional complex Lie algebra $\mathfrak{g}$. Let $\mathrm{Q}$ be the quadratic element given by $\mathrm{Q}=\sum_{i, j} d_{i} \bar{a}_{i j} H_{\alpha_{i}} \otimes H_{\alpha_{j}}$ where $\left(\bar{a}_{i j}\right)$ is the inverse of the Cartan matrix. In the basis $\left\{H_{\alpha_{i}^{*}}\right\}_{i=1 \cdots n}$ of $\mathfrak{H}$ dual to $\left\{\alpha_{i}\right\}_{i=1 \cdots n}$, the form $\mathrm{Q}$ is given by $\sum_{i, j} d_{i} a_{i j} H_{\alpha_{i}^{*}} \otimes H_{\alpha_{j}^{*}}$. So $\mathcal{H}$ and $\check{\mathcal{R}} \in \mathcal{U} \otimes \mathcal{U}$ are given by

$$
\mathcal{H}=\xi^{\sum_{i, j} d_{i} a_{i j} H_{\alpha_{i}^{*}} \otimes H_{\alpha_{j}^{*}}}, \quad \check{\mathcal{R}}=\prod_{i=1}^{N}\left(\sum_{j=0}^{\ell-1} \frac{\left(\left(q_{\beta_{i}}-q_{\beta_{i}}^{-1}\right) X_{\beta_{i}} \otimes X_{-\beta_{i}}\right)^{j}}{\left[j ; q_{\beta_{i}}^{-2}\right] !}\right),
$$

where $q_{\beta_{i}}=\xi^{\mathrm{B}\left(\beta_{i}, \beta_{i}\right) / 2}$. In [12], $\check{\mathcal{R}}$ is shown to satisfy Relations ( $\left.\check{\mathrm{R}} 1\right)$ -

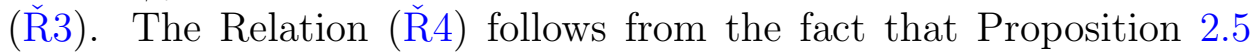
holds for quantum groups as shown in [13, Theorem 19]: in particular 
this implies that $\theta_{V}^{\prime}=\theta_{V}$ for any module $V \in \mathscr{C}^{H}$ (see Equation (11)). This equality can be interpreted as follows. Let $x$ (resp. $x^{\prime}$ ) be the element on the left (resp. right) side of Equation (ř4). Then $\theta_{V}^{\prime}=\theta_{V}$ implies that $\rho_{V}\left(x-x^{\prime}\right)=0$ where $\rho_{V}: \mathcal{U}^{H} \rightarrow \operatorname{End}_{\mathscr{C}^{H}}(V)$ is the representation $V$. Finally, Proposition 4.21 implies that $x-x^{\prime}=0$ which gives the desired result. Thus, the quantum group $\mathcal{U}$ associated to $\mathfrak{g}$ of Example 2.4 satisfies Axiom 2.

\section{Topological unRolled quantum Groups}

Unrolled quantum groups are not ribbon Hopf algebras but they admit a ribbon category of representations. The goal of this section is to define a topology on an unrolled quantum group $\mathcal{U}^{H}$ so that its completion $\widehat{\mathcal{U}^{H}}$ is a topological ribbon Hopf algebra. This topology is the one of uniform convergence on compact sets induced by the Cartan part of the algebra. The topology is nice because it produces explicit topological bases for $\widehat{\mathcal{U}^{H}}$ and allows the identification of $\mathscr{C}^{H}$ with finite dimensional $\widehat{\mathcal{U}^{H}}$ weight modules. The topological Hopf algebras are Hopf algebra objects in the category of nuclear spaces. We first recall some definitions from $[28,16,17]$.

3.1. Topological Hopf algebras. Let $E$ and $F$ be locally convex spaces. A topology is compatible with $\otimes$ if both 1$) \otimes: E \times F \rightarrow E \otimes F$ is continuous and 2) for all $(e, f) \in E^{\prime} \times F^{\prime}$ the linear form $e \otimes f$ : $E \otimes F \rightarrow \mathbb{C}, x \otimes y \mapsto e(x) f(y)$ is continuous [28, 16]. The locally convex space $E$ is called nuclear, if all the compatible topologies on $E \otimes F$ agree after completion, for all locally convex spaces $F$, i.e. the topology on the completion of $E \otimes F$ compatible with $\otimes$ is unique. For two nuclear spaces $E$ and $F$ the completion of the tensor product $E \otimes F$ endowed with its compatible topology is denoted $E \widehat{\otimes} F$. We recall some facts about nuclear spaces (see [16]): 1) a finite dimensional space is nuclear, 2) the tensor product of two nuclear spaces is a nuclear space and 3) a space is nuclear if and only if its completion is nuclear. Complete nuclear spaces form a symmetric monoidal category Nuc with the product $\widehat{\otimes}$ (see [28]). Recall we denote the flip isomorphism by $\tau: E \widehat{\otimes} F \stackrel{\sim}{\rightarrow} F \widehat{\otimes} E$.

A topological Hopf algebra is a Hopf algebra object in the monoidal category Nuc. That is a complete nuclear $\mathbb{C}$-space $\mathcal{A}$ endowed with the $\mathbb{C}$-linear maps called the product, unit, coproduct, counit and antipode $m: \mathcal{A} \widehat{\otimes} \mathcal{A} \rightarrow \mathcal{A}, \eta: \mathbb{C} \rightarrow \mathcal{A}, \Delta: \mathcal{A} \rightarrow \mathcal{A} \widehat{\otimes} \mathcal{A}, \varepsilon: \mathcal{A} \rightarrow \mathbb{C}$ and $S: \mathcal{A} \rightarrow \mathcal{A}$ which satisfy the axioms:

(1) the product $m$ is associative on $\mathcal{A}$ admitting $1_{\mathcal{A}}=\eta(1)$ as unity,

(2) $\left(\varepsilon \widehat{\otimes} \operatorname{Id}_{\mathcal{A}}\right) \circ \Delta=\left(\operatorname{Id}_{\mathcal{A}} \widehat{\otimes} \varepsilon\right) \circ \Delta=\operatorname{Id}_{\mathcal{A}}$ and the coproduct $\Delta$ is coassociative: $\left(\Delta \widehat{\otimes} \operatorname{Id}_{\mathcal{A}}\right) \circ \Delta=\left(\operatorname{Id}_{\mathcal{A}} \widehat{\otimes} \Delta\right) \circ \Delta$, 
(3) $\Delta$ and $\varepsilon$ are algebra morphisms where the associative product in $\mathcal{A} \widehat{\otimes} \mathcal{A}$ is determined by $(m \widehat{\otimes} m) \circ\left(\operatorname{Id}_{\mathcal{A}} \widehat{\otimes} \tau \widehat{\otimes} \operatorname{Id}_{\mathcal{A}}\right)$,

(4) $m \circ\left(S \widehat{\otimes} \operatorname{Id}_{\mathcal{A}}\right) \circ \Delta=m \circ\left(\operatorname{Id}_{\mathcal{A}} \widehat{\otimes} S\right) \circ \Delta=\eta \circ \varepsilon$.

If $V$ is a finite dimensional $\mathbb{C}$-vector space we denote by $\mathscr{C}^{\omega}(V)$ the space of entire functions on $V$ endowed with the topology of uniform convergence on compact sets. Then $\mathscr{C}^{\omega}(V)$ is a complete nuclear space. If $\left\{Z_{i}\right\}_{i=1, \ldots, n}$ are the coordinate functions of $V$ in some basis then we denote $\mathscr{C}^{\omega}(V)$ by $\mathscr{C}^{\omega}\left(Z_{1}, \ldots, Z_{n}\right)$. Remark that we have $\mathscr{C}^{\omega}\left(V_{1}\right) \widehat{\otimes}^{\omega} \mathscr{C}^{\omega}\left(V_{2}\right) \simeq \mathscr{C}^{\omega}\left(V_{1} \times V_{2}\right)$ where $V_{1}, V_{2}$ are finite dimensional $\mathbb{C}$-vector spaces (see [31, Theorem 51.6]).

We will need the following example later. Recall the vector spaces $W$ and $\mathfrak{H}$ given in Section 2. The nuclear space $W \widehat{\otimes} \mathscr{C}^{\omega}\left(\mathfrak{H}^{*}\right)$ can be seen as $W$-valued entire functions, whose elements are power series in the variables $\left(H_{i}\right)_{i=1 \cdots n}$ with coefficients in $W$. Alternatively, if $\mathfrak{B}_{W}$ is a base of $W$, one can think of $W \widehat{\otimes} \mathscr{C}^{\omega}\left(\mathfrak{H}^{*}\right)$ as the span of $\mathfrak{B}_{W}$ with coefficients in $\mathscr{C}^{\omega}\left(\mathfrak{H}^{*}\right)$.

Proposition 3.1 ([17]). Let $\mathfrak{H}_{1}, \mathfrak{H}_{2}, W_{1}, W_{2}$ be finite dimensional $\mathbb{C}$ vector spaces. Then

$$
\left(W_{1} \otimes \mathscr{C}^{\omega}\left(\mathfrak{H}_{1}^{*}\right)\right) \widehat{\otimes}\left(W_{2} \otimes \mathscr{C}^{\omega}\left(\mathfrak{H}_{2}^{*}\right)\right) \simeq\left(W_{1} \otimes W_{2}\right) \otimes \mathscr{C}^{\omega}\left(\mathfrak{H}_{1}^{*} \times \mathfrak{H}_{2}^{*}\right) .
$$

3.2. Topology on the completion of $\mathcal{U}^{H}$. The space of entire functions is a nuclear space obtained as the completion of polynomial functions for the topology of uniform convergence on compact sets. We use a similar completion to define a topological ribbon Hopf algebra from $\mathcal{U}^{H}$.

Recall that as a vector space,

$$
\mathcal{U}^{H}=W \otimes_{\mathbb{C}} \mathbb{C}\left[\Lambda^{*}\right] \otimes_{\mathbb{C}} S \mathfrak{H}
$$

where $W$ is a finite dimensional $\mathbb{C}$-vector space, $\mathbb{C}\left[\Lambda^{*}\right]=\mathbb{C}\left[K_{1}^{ \pm 1}, \ldots, K_{r}^{ \pm 1}\right]$ is the space of Laurent polynomials in $r$ variables and $S \mathfrak{H}=\mathbb{C}\left[H_{1}, \ldots, H_{r}\right]$ is the space of polynomials in $r$ variables (here we write $H_{i}$ for $H_{a_{i}}$ and $K_{i}$ for $K^{a_{i}}$, where $\left(a_{i}\right)_{i}$ is the fixed basis of $\left.\Lambda^{*}\right)$.

Denote $\mathscr{C}^{\omega}\left(\mathfrak{H}^{*}\right)$ by $\mathscr{C}^{\omega}\left(H_{1}, \ldots, H_{r}\right)$. We embed the commutative algebra $\mathbb{C}\left[\Lambda^{*}\right] \otimes_{\mathbb{C}} S \mathfrak{H}$ into $\mathscr{C}^{\omega}\left(H_{1}, \ldots, H_{r}\right)$ by seeing $H_{i}$ as a linear function on $\mathfrak{H}^{*}$ and by sending $K_{i}=K^{a_{i}}$ to $\xi^{H_{i}}=\exp \left(\frac{2 i \pi}{\ell} H_{i}\right)$. Then $\mathcal{U}^{H}$ is embedded in $W \otimes_{\mathbb{C}} \mathscr{C}^{\omega}\left(\mathfrak{H}^{*}\right)$.

Now we put on $\mathscr{C}^{\omega}\left(\mathfrak{H}^{*}\right)$ the topology of uniform convergence on compact sets. Then it is a complete nuclear space and as $W$ is finite dimensional, one has

$$
W \otimes_{\mathbb{C}} \mathscr{C}^{\omega}\left(\mathfrak{H}^{*}\right)=W \widehat{\otimes}_{\mathbb{C}} \mathscr{C}^{\omega}\left(\mathfrak{H}^{*}\right) .
$$

Furthermore the embedding of $\mathcal{U}^{H}$ in this complete nuclear space is dense in it so we have:

$$
\widehat{\mathcal{U}^{H}}=W \widehat{\otimes} \mathscr{C}^{\omega}\left(\mathfrak{H}^{*}\right) \simeq W \otimes_{\mathbb{C}} \mathscr{C}^{\omega}\left(H_{1}, \ldots, H_{r}\right) .
$$


Let us now describe a family of semi-norms that generates the topology of $\widehat{\mathcal{U}^{H}}$. Any two norms on $W$ are equivalent. Let choose a norm $\|\cdot\|$ on $W$. Then for any compact set $C$ in $\mathfrak{H}^{*}$, we have a semi-norm $\|\cdot\|_{C}$ on $W \otimes_{\mathbb{C}} \mathscr{C}^{\omega}\left(\mathfrak{H}^{*}\right)$ defined as follows: If $\varphi \in C$ and $x$ is a multivariable power series such that $x\left(H_{1}, \ldots, H_{r}\right) \in \mathscr{C}^{\omega}\left(\mathfrak{H}^{*}\right)$ then $\varphi_{*} x\left(H_{1}, \ldots, H_{r}\right)$ is the evaluation of $x$ at $\varphi$, that is $\varphi_{*} x\left(H_{1}, \ldots, H_{r}\right)=x\left(\varphi\left(H_{1}\right), \ldots, \varphi\left(H_{r}\right)\right) \in$ $\mathbb{C}$. Then for $u=\sum_{k} w_{k} x_{k}\left(H_{1}, \ldots, H_{r}\right) \in \widehat{\mathcal{U}^{H}}$ the semi-norm of $u$ associated to $C$ is defined by

$$
\|u\|_{C}=\sup _{\varphi \in C}\left\|\varphi_{*} u\right\|
$$

where $\varphi_{*} u=\sum_{k} w_{k} \varphi_{*} x_{k} \in W$. One easily sees that $\|\cdot\|_{C}$ does not depend of the norm on $W$ up to equivalence. The family of seminorms $\left\{\|\cdot\|_{C}\right\}_{C \text { compact }}$ generates the topology of uniform convergence on compact sets of $W$-valued entire functions.

The rest of this subsection is dedicated to proving that the complete nuclear space $\widehat{\mathcal{U}^{H}}$ has a natural structure of a topological ribbon Hopf algebra. This means that $\widehat{\mathcal{U}^{H}}$ is a topological Hopf algebra with an invertible element $\mathcal{R} \in \mathcal{U}^{H} \widehat{\otimes} \mathcal{U}^{H}$ called the universal $R$-matrix and a central element $\theta \in \widehat{\mathcal{U}^{H}}$ called the twist ${ }^{2}$

$$
\begin{aligned}
\mathcal{R} \Delta(x) & =\Delta^{o p}(x) \mathcal{R} \text { for all } x \in \mathcal{U}^{H}, \\
\Delta \otimes \operatorname{Id}(\mathcal{R}) & =\mathcal{R}_{13} \mathcal{R}_{23}, \\
\operatorname{Id} \otimes \Delta(\mathcal{R}) & =\mathcal{R}_{13} \mathcal{R}_{12}, \\
S(\theta) & =\theta, \\
\Delta(\theta) & =\mathcal{R}_{21} \mathcal{R}(\theta \otimes \theta), \\
\varepsilon(\theta) & =1 .
\end{aligned}
$$

Recall the element $\mathrm{Q}=\sum_{i} c_{i} \otimes c_{i}^{\prime} \in \mathfrak{H} \otimes \mathfrak{H}$ and define

$$
\mathcal{H}=\xi^{\mathrm{Q}} \in \mathcal{U}^{H} \widehat{\otimes} \mathcal{U}^{H} \text {. }
$$

Theorem 3.2. $\widehat{\mathcal{U}^{H}}$ is a topological ribbon Hopf algebra with universal $R$-matrix

$$
\mathcal{R}=\mathcal{H} \check{\mathcal{R}} \in \mathcal{U}^{H} \widehat{\otimes} \mathcal{U}^{H}
$$

and twist

$$
\theta=g^{-1}\left(m \circ\left(S^{2} \otimes \operatorname{Id}\right)(\mathcal{R})\right) \in \widehat{\mathcal{U}^{H}} .
$$

Remark that as for ribbon Hopf algebras, following the lines of [21, $\S \mathrm{VIII}]$ we can prove that $\left(\widehat{\mathcal{U}^{H}}, \mathcal{R}, \theta\right)$ satisfies the following additional properties:

$$
\begin{aligned}
\mathcal{R}_{12} \mathcal{R}_{13} \mathcal{R}_{23} & =\mathcal{R}_{23} \mathcal{R}_{13} \mathcal{R}_{12}, & (\varepsilon \otimes \operatorname{Id})(\mathcal{R}) & =1=(\operatorname{Id} \otimes \varepsilon)(\mathcal{R}), \\
(S \otimes \operatorname{Id})(\mathcal{R}) & =\mathcal{R}^{-1}=\left(\operatorname{Id} \otimes S^{-1}\right)(\mathcal{R}), & \mathcal{R} & =(S \otimes S)(\mathcal{R}) .
\end{aligned}
$$

\footnotetext{
${ }^{2}$ In the literature the inverse of the element $\theta$ is often considered.
} 
To prove the theorem, we need the following three lemmas which imply that the product, coproduct and antipode are continuous.

As $W$ is finite dimensional, all norms on $W$ are equivalent. Nevertheless to prove the theorem, we fix a convenient choice of norm as follows: let $\mathfrak{B}_{W}=\left(w_{1}, \ldots, w_{n}\right)$ be a basis of $W$ where $w_{i}$ is homogeneous of degree $\lambda_{i} \in \Lambda$. Then let $\|\cdot\|$ be the maximum norm in $\mathfrak{B}_{W}$, which means $\left\|\sum_{i} x_{i} w_{i}\right\|=\sup _{i}\left|x_{i}\right|$. Similarly, $\mathfrak{B}_{W}^{\otimes k}$ is a basis of $W^{\otimes k}$ that will be equipped with the associated maximum norm. Then one easily checks that $\left\|w \otimes w^{\prime}\right\|=\|w\|\left\|w^{\prime}\right\|$.

Let $E, F$ be nuclear spaces and let $\mathcal{N}_{F}$ be a set of semi-norms on $F$ that generate its topology. Then remark that a linear map $f: E \rightarrow F$ is continuous if and only if for any continuous semi-norm $\|\cdot\|_{F} \in \mathcal{N}_{F}$, there exists a continuous norm $\|\cdot\|_{E}$ on $E$ and a constant $\eta \in \mathbb{R}^{+}$such that

$$
\forall x \in E,\|f(x)\|_{F} \leq \eta\|x\|_{E} .
$$

Lemma 3.3. For each compact set $C \subset \mathfrak{H}^{*}$, there exists a compact set $C^{\prime}$ and a constant $\lambda_{C} \in \mathbb{R}$ such that $\forall x, y \in \mathcal{U}^{H}$, we have

$$
\|x y\|_{C} \leq \lambda_{C}\|x \otimes y\|_{C^{\prime} \times C} .
$$

Proof. Let $\Lambda_{W}=\left\{\lambda_{i}, i=1 \cdots N\right\}$ which is a finite set and $C^{\prime}=C+\Lambda_{W}$ which is also compact. Then we write

$$
\begin{gathered}
\varphi_{*}(x y)=\varphi_{*}\left(\sum_{i} w_{i} x_{i}\left(H_{1}, \ldots, H_{r}\right) \sum_{j} w_{j} y_{j}\left(H_{1}, \ldots, H_{r}\right)\right) \\
=\varphi_{*}\left(\sum_{i, j} w_{i} w_{j} x_{i}\left(H_{1}+a_{1}\left(\lambda_{j}\right), \ldots, H_{r}+a_{r}\left(\lambda_{j}\right)\right) y_{j}\left(H_{1}, \ldots, H_{r}\right)\right) \\
=\sum_{i, j} \varphi_{*}\left(w_{i} w_{j}\right) \cdot\left(\varphi+\lambda_{j}\right)_{*}\left(x_{i}\right) \cdot \varphi_{*}\left(y_{j}\right) .
\end{gathered}
$$

Then

$$
\begin{aligned}
\|x y\|_{C} & =\sup _{\varphi \in C}\left\|\varphi_{*}(x y)\right\| \leq \sup _{\varphi \in C} \sum_{i, j}\left\|\varphi_{*}\left(w_{i} w_{j}\right)\right\| \cdot\left|\left(\varphi+\lambda_{j}\right)_{*}\left(x_{i}\right)\right| \cdot\left|\varphi_{*}\left(y_{j}\right)\right| \\
& \leq \sum_{i, j} \sup _{\varphi \in C}\left\|\varphi_{*}\left(w_{i} w_{j}\right)\right\| \sup _{\varphi \in C^{\prime}}\left|\varphi_{*}\left(x_{i}\right)\right| \sup _{\varphi \in C}\left|\varphi_{*}\left(y_{j}\right)\right| \\
& \leq\|x\|_{C^{\prime}}\|y\|_{C} \sum_{i, j}\left\|w_{i} w_{j}\right\|_{C},
\end{aligned}
$$

and one can take $\lambda_{C}=\sum_{i, j}\left\|w_{i} w_{j}\right\|_{C}$ so that $\|x y\|_{C} \leq \lambda_{C}\|x\|_{C^{\prime}}\|y\|_{C}=$ $\lambda_{C}\|x \otimes y\|_{C^{\prime} \times C}$.

By Proposition 3.1 we have $\mathcal{U}^{H} \widehat{\otimes} \mathcal{U}^{H} \simeq W^{\otimes 2} \otimes \mathscr{C}^{\omega}\left(H_{i, j}\right)$ where $H_{i, 1}=$ $H_{i} \otimes 1, H_{i, 2}=1 \otimes H_{i}$ for $i=1, \ldots, r$ and the $H_{i, j}$ are seen as coordinates 
functions on $\mathfrak{H}^{*} \times \mathfrak{H}^{*}$. If $C_{2} \subset \mathfrak{H}^{*} \times \mathfrak{H}^{*}$ is compact, we have the associated seminorm

$$
\|x\|_{C_{2}}=\sup _{\varphi \in C_{2}}\left\|\varphi_{*} x\right\|
$$

where $\|\cdot\|$ is the maximum norm in the basis $\mathfrak{B}_{W}^{\otimes 2}$ of $W^{\otimes 2}$.

Lemma 3.4. For each compact set $C_{2} \subset \mathfrak{H}^{*} \times \mathfrak{H}^{*}$, there exists a compact set $C \subset \mathfrak{H}^{*}$ and $\lambda_{C_{2}} \in \mathbb{R}$ such that $\forall x \in \mathcal{U}^{H}$, we have

$$
\|\Delta x\|_{C_{2}} \leq \lambda_{C_{2}}\|x\|_{C} \text {. }
$$

Proof. Let $\sigma: \mathfrak{H}^{*} \times \mathfrak{H}^{*} \rightarrow \mathfrak{H}^{*}$ be the sum $\left(\varphi, \varphi^{\prime}\right) \mapsto \varphi+\varphi^{\prime}$ and $C=\sigma\left(C_{2}\right)$ which is a compact in $\mathfrak{H}^{*}$. Let $x=\sum_{i} w_{i} x_{i}\left(H_{1}, \ldots, H_{r}\right)$ be written in the basis $\mathfrak{B}_{W}$, then

$$
\begin{aligned}
& \|\Delta x\|_{C_{2}}=\left\|\sum_{i}\left(\Delta w_{i}\right) x_{i}\left(H_{1,1}+H_{1,2}, \ldots, H_{r, 1}+H_{r, 2}\right)\right\|_{C_{2}} \\
\leq & \sum_{i} \sup _{\varphi \in C_{2}}\left\|\varphi_{*} \Delta w_{i}\right\| \sup _{\varphi \in C_{2}}\left|\varphi_{*} x_{i}\left(H_{1,1}+H_{1,2}, \ldots, H_{r, 1}+H_{r, 2}\right)\right| \\
\leq & \sum_{i}\left\|\Delta w_{i}\right\|_{C_{2}} \sup _{\varphi \in C}\left|\varphi_{*} x_{i}\left(H_{1}, \ldots, H_{r}\right)\right| \leq\|x\|_{C} \sum_{i}\left\|\Delta w_{i}\right\|_{C_{2}},
\end{aligned}
$$

and one can take $\lambda_{C_{2}}=\sum_{i}\left\|\Delta w_{i}\right\|_{C_{2}}$.

Lemma 3.5. For each compact set $C \subset \mathfrak{H}^{*}$ there exists a compact set $C^{\prime} \subset \mathfrak{H}^{*}$ and a constant $\lambda_{C}$ such that

$$
\|S(x)\|_{C} \leq \lambda_{C}\|x\|_{C^{\prime}} \text { for } x \in \mathcal{U}^{H} .
$$

Proof. Let $C^{\prime}=-\Lambda_{W}-C=\bigcup_{i}-\left(\lambda_{i}+C\right)$ which is compact. Let $x=\sum_{i} w_{i} x_{i}\left(H_{1}, \ldots, H_{r}\right)$ be written in the basis $\mathfrak{B}_{W}$, then

$$
\begin{aligned}
\|S(x)\|_{C} & =\left\|\sum_{i} x_{i}\left(-H_{1}, \ldots,-H_{r}\right) S\left(w_{i}\right)\right\|_{C} \\
& \leq\left\|\sum_{i} S\left(w_{i}\right) x_{i}\left(-a_{1}\left(\lambda_{i}\right)-H_{1}, \ldots,-a_{r}\left(\lambda_{i}\right)-H_{r}\right)\right\|_{C} \\
& \leq\|x\|_{C^{\prime}} \sum_{i}\left\|S\left(w_{i}\right)\right\|_{C} .
\end{aligned}
$$

Proof of Theorem 3.2. Recall $\widehat{\mathcal{U}^{H}}$ is the completion of $\mathcal{U}^{H}$. Therefore, to show that $\widehat{\mathcal{U}^{H}}$ is a topological Hopf algebra it is enough to show the structure morphisms of the Hopf algebra $\mathcal{U}^{H}$ are continuous for the topology of uniform convergence on compact sets: This is obvious for the unit and follows for the counit as $\varepsilon\left(x\left(H_{1}, \ldots, H_{r}\right)\right)=x(0, \ldots, 0)$. Lemmas 3.3, 3.4 and 3.5 combined with Equation (17) imply the continuity for the product, the coproduct and the antipode, respectively. 
Finally, we need to show that $\mathcal{R}$ is a universal $\mathrm{R}$-matrix and $\theta$ is a twist. Properties (R1)-(R3) follow from combining the fact that $\widetilde{\mathcal{H}}$ is the conjugation by $\mathcal{H}$ and $\widetilde{\mathcal{R}}$ satisfies Relations ( $\mathrm{R} 1)-(\check{\mathrm{R}} 3)$.

Next we prove Axiom (R4). Let $u=\sum_{i} S\left(b_{i}\right) a_{i} \in \widehat{\mathcal{U}^{H}}$ be the Drinfeld element where $\mathcal{R}=\sum_{i} a_{i} \otimes b_{i} \in \mathcal{U}^{H} \widehat{\otimes} \mathcal{U}^{H}$. Then following [21, §VIII] we have $u$ is invertible with inverse $u^{-1}=\sum_{i} b_{i} S^{2}\left(a_{i}\right)$. Then

$$
S\left(u^{-1}\right)=\sum_{i} S^{3}\left(a_{i}\right) S\left(b_{i}\right)=\sum_{i} S^{2}\left(a_{i}\right) b_{i}=g \theta .
$$

But $\mathcal{R}=\mathcal{H} \check{\mathcal{R}}$ where $\check{\mathcal{R}}=\sum_{i} x_{i} \otimes y_{i} \in \mathcal{U} \otimes \mathcal{U}$. Then (recall $x_{i}$ and $y_{i}$ have opposite degree)

$$
\begin{aligned}
\mathcal{R} & =\sum_{i} \widetilde{\mathcal{H}}\left(1 \otimes y_{i}\right) \cdot \mathcal{H} \cdot\left(x_{i} \otimes 1\right)=\sum_{i}\left(B_{\left|x_{i}\right|}^{-1} \otimes y_{i}\right) \cdot \mathcal{H} \cdot\left(x_{i} \otimes 1\right) \\
& =\sum_{i}\left(1 \otimes y_{i}\right) \cdot \mathcal{H} \cdot\left(B_{\left|x_{i}\right|}^{-1} x_{i} \otimes 1\right)
\end{aligned}
$$

and similarly

$$
\mathcal{R}=\sum_{i}\left(x_{i} \otimes 1\right) \cdot \mathcal{H} \cdot\left(1 \otimes B_{\left|x_{i}\right|} y_{i}\right)
$$

thus from (19),

$$
S\left(u^{-1}\right)=m(\mathcal{H}) \sum_{i} B_{\left|x_{i}\right|}^{-1} S^{2}\left(x_{i}\right) y_{i}=m(\mathcal{H}) g \sum_{i} x_{i} g^{-1} y_{i} B_{\left|x_{i}\right|}^{-1}
$$

and from (20), one gets

$$
u^{-1}=m(\mathcal{H}) \sum_{i} B_{\left|x_{i}\right|} y_{i} S^{2}\left(x_{i}\right)=m(\mathcal{H}) g^{-1} \sum_{i} y_{i} g x_{i} B_{\left|x_{i}\right|} .
$$

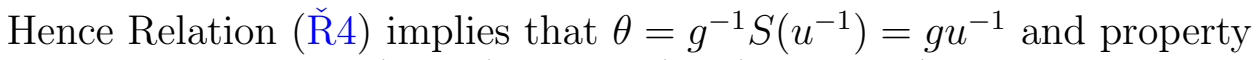
(R4) follows from $g^{-1} S\left(u^{-1}\right)=S\left(u^{-1}\right) g^{-1}=S\left(g u^{-1}\right)=S(\theta)$.

To prove the last two equalities we follow the proof given in [21, $\S$ VIII] for braided Hopf algebras. In particular, the element $u$ satisfies

$$
\Delta(u)=\left(\mathcal{R}_{21} \mathcal{R}\right)^{-1}(u \otimes u)=(u \otimes u)\left(\mathcal{R}_{21} \mathcal{R}\right)^{-1} \quad \text { and } \quad \varepsilon(u)=1 .
$$

Then as $\theta=g u^{-1}$, we have

$$
\begin{aligned}
\Delta(\theta)=\Delta\left(g u^{-1}\right) & =\Delta(g) \Delta\left(u^{-1}\right) \\
& =(g \otimes g)\left(\left(\mathcal{R}_{21} \mathcal{R}\right)^{-1}(u \otimes u)\right)^{-1} \\
& =(g \otimes g)\left(u^{-1} \otimes u^{-1}\right)\left(\mathcal{R}_{21} \mathcal{R}\right) \\
& =(\theta \otimes \theta)\left(\mathcal{R}_{21} \mathcal{R}\right)=\left(\mathcal{R}_{21} \mathcal{R}\right)(\theta \otimes \theta) .
\end{aligned}
$$

This proves Equation (R5). The proof of Equation (R6) is similar. 
3.3. Category of module. Let $\widehat{\mathcal{U}}^{H}$-mod be the category of topological modules (i.e. object of the category of complete nuclear space equipped with $\widehat{\mathcal{U}}^{H}$-module maps in this catetegory).

Proposition 3.6. $\mathscr{C}^{H}$ is a full subcategory of $\widehat{\mathcal{U}^{H}}-$ mod.

Proof. Since a module $V$ of $\mathscr{C}^{H}$ is finite dimensional, its set of weights is compact and the action of $\mathcal{U}^{H}$ :

$$
\rho_{V}: \mathcal{U}^{H} \otimes V \rightarrow V
$$

is continuous and extend to a continuous map $\rho_{V}: \widehat{\mathcal{U}^{H}} \otimes V \rightarrow V$.

3.4. Power elements. Recall

$$
\begin{aligned}
\Lambda & \simeq \mathbb{Z}^{r}, & \Lambda^{*} & =\operatorname{Hom}_{\mathbb{Z}}(\Lambda, \mathbb{Z})=\oplus_{i} \mathbb{Z} H_{i}, \\
\mathfrak{H}^{*} & =\Lambda \otimes_{\mathbb{Z}} \mathbb{C}, & \mathfrak{H} & =\Lambda^{*} \otimes_{\mathbb{Z}} \mathbb{C}=\oplus_{i} \mathbb{C} H_{i} .
\end{aligned}
$$

Let

$$
G=\mathfrak{H}^{*} / \Lambda \simeq(\mathbb{C} / \mathbb{Z})^{r} \quad \text { and } \quad G^{\prime}=\mathfrak{H} / \Lambda^{*} \simeq(\mathbb{C} / \mathbb{Z})^{r} .
$$

Also, let

$\mathfrak{H}^{(n)}=(\mathfrak{H} \otimes 1 \otimes \cdots \otimes 1) \oplus(1 \otimes \mathfrak{H} \otimes 1 \otimes \cdots \otimes 1) \oplus \cdots \oplus(1 \otimes \cdots \otimes 1 \otimes \mathfrak{H})$ which is a subset of $(S \mathfrak{H})^{\otimes n} \subset\left(\mathcal{U}^{H}\right)^{\otimes n}$. The elements of $\mathfrak{H}^{(n)}$ are called linear. For $i=1 \cdots r$ and $j=1 \cdots n$ let $H_{i, j}$ be the element of $\mathfrak{H}^{(n)}$ which is $H_{i}$ in the $j$ th direct sum (or tensor product) of $\mathfrak{H}^{(n)}$. The elements $\left\{H_{i, j}: i=1 \cdots r, j=1 \cdots n\right\}$ form a basis of $\mathfrak{H}^{(n)}$. Recall $\widehat{\mathcal{U}^{H}} \simeq W \otimes_{\mathbb{C}} \mathscr{C}^{\omega}\left(H_{1}, \ldots, H_{r}\right)$ and it follows that

$$
\left(\mathcal{U}^{H}\right)^{\widehat{\otimes} n} \simeq \mathscr{C}^{\omega}\left(H_{i, j}\right) \otimes_{\mathbb{C}} W^{\otimes n} .
$$

An element of $\left(\mathcal{U}^{H}\right)^{\widehat{\otimes} n}$ the form $\xi^{H}$ for some $H \in \mathfrak{H}^{(n)}$ is called power linear. The set $\mathcal{L}_{n}$ of power linear elements of $\left(\mathcal{U}^{H}\right)^{\widehat{\otimes} n}$ form a commutative Lie group among invertible elements of $\left(\mathcal{U}^{H}\right)^{\widehat{\otimes} n}$. Furthermore, we have the following two facts: 1 ) the elements of $\mathcal{L}_{1}$ are group-like and 2) $\mathcal{L}_{n}=\left(\mathcal{L}_{1}\right)^{\otimes n}$. The complex vector space $\mathfrak{H}^{(n)}$ contains a $\mathbb{Z}$ lattice $\Lambda^{*(n)}=\bigoplus_{i, j} \mathbb{Z} H_{i, j}$. The exponential of elements of $\Lambda^{*(n)}$ form a multiplicative subgroup of $\mathcal{L}_{n}$ generated by the elements $K_{i, j}=\xi^{H_{i, j}}$.

We say a finite sum $\sum_{\alpha} H_{\alpha} H_{\alpha}^{\prime}$ in $\left(\mathcal{U}^{H}\right)^{\otimes n}$, for some $H_{\alpha}, H_{\alpha}^{\prime} \in \Lambda^{*(n)}$ is quadratic. Similarly, elements of the set

$$
\mathcal{Q}_{n}=\left\{\xi^{q}: q \text { in }\left(\mathcal{U}^{H}\right)^{\otimes n} \text { is quadratic }\right\}
$$

are called power quadratic. For example, the element $\mathcal{H}=\xi^{\mathrm{Q}}$ is a power quadratic element in $\mathcal{Q}_{2}$.

Let us write $h_{\bullet}=\left\{H_{i, j}\right\}$ for the vector of coordinates functions. If $q\left(h_{\bullet}\right)$ is a quadratic element then let

$$
\widetilde{q}\left(h_{\bullet}, h_{\bullet}^{\prime}\right)=q\left(h_{\bullet}+h_{\bullet}^{\prime}\right)-q\left(h_{\bullet}\right)-q\left(h_{\bullet}^{\prime}\right)
$$


be the associated integral symmetric bilinear form such that $\widetilde{q}\left(h_{\bullet}, h_{\bullet}\right)=$ $2 q\left(h_{\bullet}\right)$. For example, if $q\left(h_{\bullet}\right)=H_{1,1} H_{3,2}=H_{1} \otimes H_{3}$ and $q\left(h_{\bullet}^{\prime}\right)=$ $H_{1,1}^{\prime} H_{3,2}^{\prime}$ are in $\Lambda^{*(2)}$ then

$$
\begin{aligned}
\widetilde{q}\left(h_{\bullet}, h_{\bullet}^{\prime}\right) & =\left(H_{1,1}+H_{1,1}^{\prime}\right)\left(H_{3,2}+H_{3,2}^{\prime}\right)-H_{1,1} H_{3,2}-H_{1,1}^{\prime} H_{3,2}^{\prime} \\
& =H_{1,1} H_{3,2}^{\prime}+H_{1,1}^{\prime} H_{3,2} .
\end{aligned}
$$

Remark that the notation $q\left(h_{\bullet}, h_{\bullet}^{\prime}\right)$ makes easy the change of variables. As such a function can be interpreted as a function on $\left(\mathfrak{H}^{(n)}\right)^{*} \times\left(\mathfrak{H}^{(n)}\right)^{*}$, if $\omega \in\left(\mathfrak{H}^{(n)}\right)^{*}$, we denote $q\left(\omega, h_{\bullet}^{\prime}\right)$ the function $q\left(h_{\bullet}, h_{\bullet}^{\prime}\right)$ where the variables $h_{\bullet}$ has been evaluated with $\omega$ i.e., we substitute $H_{i, j}$ with $\omega\left(H_{i, j}\right) \in \mathbb{C}$.

Since $q$ is an integral linear combination of the elements $H_{i, j}$, we have

Lemma 3.7. Let $q \in\left(\mathcal{U}^{H}\right)^{\otimes n}$ be quadratic. We have (1) if $\omega \in$ $\left(\mathfrak{H}^{(n)}\right)^{*} \simeq\left(\mathfrak{H}^{*}\right)^{n}$ then $\widetilde{q}\left(\omega, h_{\bullet}\right)$ defines an element of $\mathfrak{H}^{(n)}$, (2) furthermore, if $\omega \in \Lambda^{n}$, then $\widetilde{q}(\omega, h \bullet) \in\left(\Lambda^{*}\right)^{n}$ and (3) q induces a map $G^{n} \rightarrow G^{\prime n}$ given by

$$
\bar{\omega} \mapsto \widetilde{q}\left(\bar{\omega}, h_{\bullet}\right) \in\left(G^{\prime}\right)^{n}
$$

Remark 3.8. If $l$ is a linear element, the space $\xi^{l} \mathcal{U}^{\otimes n} \subset \mathcal{L}_{n} \mathcal{U}^{\otimes n}$ only depends on the class $\bar{l}$ of $l$ in $\left(G^{\prime}\right)^{n}$. Indeed, if $l^{\prime}$ and $l$ are in the same class in $\left(G^{\prime}\right)^{n}$ then $\xi^{l^{\prime}-l}$ is a unit in $\mathcal{U}^{\otimes n}$ so $\xi^{l^{\prime}-l} \mathcal{U}^{\otimes n}=\mathcal{U}^{\otimes n}$. In particular, if $q$ is quadratic and $\bar{\omega} \in G^{n}$ then $\xi^{\widetilde{q}\left(\bar{\omega}, h_{\bullet}\right)} \mathcal{U}^{\otimes n}$ is the space $\xi^{l} \mathcal{U}^{\otimes n}$ for any representant $l$ of $\widetilde{q}\left(\bar{\omega}, h_{\bullet}\right) \in\left(G^{\prime}\right)^{n}$.

The following lemma generalizes the conjugation by $\mathcal{H}$ given by Equation (8):

Lemma 3.9. Let $L=\xi^{l} \in \mathcal{L}_{n}$ and $Q=\xi^{q} \in \mathcal{Q}_{n}$ where $l \in \mathfrak{H}^{(n)}$ is a linear element and $q \in\left(\mathcal{U}^{H}\right)^{\otimes n}$ is quadratic. Let $x$ be an homogeneous element of $\left(\mathcal{U}^{H}\right)^{\widehat{\otimes} n}$ of weight $\lambda \in \Lambda^{n}$. Then

$$
L x L^{-1}=\lambda_{*}(L) x,
$$

and for $K=\xi^{\widetilde{q}\left(\lambda, h_{\bullet}\right)} \in \xi^{\Lambda^{*(n)}} \subset \mathcal{U}^{\otimes n}$ we have

$$
Q x Q^{-1}=\lambda_{*}(Q) x K .
$$

Proof. Recall we write $h_{\bullet}=\left\{H_{i, j}\right\}, L=\xi^{l\left(h_{\bullet}\right)}$ and $Q=\xi^{q\left(h_{\bullet}\right)}=$

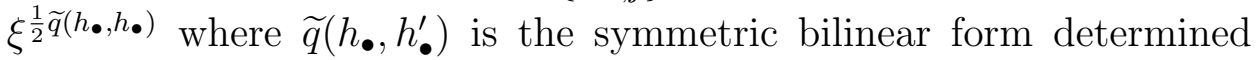
by $q$. Then from Equation (3) we have

$$
\begin{aligned}
& L x=\xi^{l\left(h_{\bullet}\right)} x=x \xi^{l\left(h_{\bullet}+\lambda\right)}=x \xi^{l\left(h_{\bullet}\right)} \xi^{l(\lambda)}=x L \lambda_{*}(L), \quad \text { and } \\
& Q x=x \xi^{q\left(h_{\bullet}+\lambda\right)}=x \xi^{q\left(h_{\bullet}\right)} \xi^{q(\lambda)} \xi^{\widetilde{q}\left(\lambda, h_{\bullet}\right)}=x Q \lambda_{*}(Q) \xi^{\widetilde{q}\left(\lambda, h_{\bullet}\right)} .
\end{aligned}
$$

The result follows because $Q$ commutes with $\xi^{\widetilde{q}\left(\lambda, h_{\bullet}\right)}$ as they are power elements. 
The following proposition records some properties of power elements, the proof follows directly from the previous lemma and the definitions of power elements.

Proposition 3.10. (1) We have inclusions of algebras:

$$
\mathcal{U}^{\otimes n} \subset\left\langle\mathcal{Q}_{n} \mathcal{U}^{\otimes n}\right\rangle \subset\left\langle\mathcal{Q}_{n} \mathcal{L}_{n} \mathcal{U}^{\otimes n}\right\rangle \subset\left(\mathcal{U}^{H}\right)^{\widehat{\otimes} n}
$$

where $\langle X\rangle$ means linear combinations of elements of $X$.

(2) The sets $\mathcal{Q}_{n} \mathcal{U}^{\otimes n}$ and $\mathcal{Q}_{n} \mathcal{L}_{n} \mathcal{U}^{\otimes n}$ are stable by permutation of the factors, by the maps $S_{i}=\operatorname{Id} \otimes S \otimes \operatorname{Id}$ (antipode at the $i^{\text {th }}$ position).

(3) For $\Delta_{i}=\mathrm{Id} \otimes \Delta \otimes \mathrm{Id}$ and $m_{i}=\mathrm{Id} \otimes m \otimes \mathrm{Id}$, we have

$$
\begin{aligned}
& \Delta_{i}\left(\mathcal{Q}_{n} \mathcal{U}^{\otimes n}\right) \subset \mathcal{Q}_{n+1} \mathcal{U}^{\otimes n+1} \text { and } \Delta_{i}\left(\mathcal{Q}_{n} \mathcal{L}_{n} \mathcal{U}^{\otimes n}\right) \subset \mathcal{Q}_{n+1} \mathcal{L}_{n+1} \mathcal{U}^{\otimes n+1}, \\
& m_{i}\left(\mathcal{Q}_{n+1} \mathcal{U}^{\otimes n+1}\right) \subset \mathcal{Q}_{n} \mathcal{U}^{\otimes n} \text { and } m_{i}\left(\mathcal{Q}_{n+1} \mathcal{L}_{n+1} \mathcal{U}^{\otimes n+1}\right) \subset \mathcal{Q}_{n} \mathcal{L}_{n} \mathcal{U}^{\otimes n} .
\end{aligned}
$$

(4) If $V \in \mathscr{C}^{H}$ is an homogeneous weight module then

$$
\operatorname{Id}^{\otimes n} \otimes \rho_{V}\left(\mathcal{Q}_{n+1} \mathcal{L}_{n+1} \mathcal{U}^{\otimes n+1}\right) \subset \mathcal{Q}_{n} \mathcal{L}_{n} \mathcal{U}^{\otimes n} \otimes_{\mathbb{C}} \operatorname{End}_{\mathbb{C}}(V) .
$$

\section{G-CoAlgebra And Discrete Fourier transforms}

In this section we continue an algebraic investigation of $\mathcal{U}$. The results of this section will not be used until Section 6. In particular, Section 5 can be read independently.

4.1. Pivotal Hopf $G$-coalgebra from $\mathcal{U}$. We now recall the definition of Hopf group-coalgebra from [32, 36]. For coherence we will keep an additive notation for the group $G^{3}$. A Hopf $G$-coalgebra is a family $H=\left\{H_{\alpha}\right\}_{\alpha \in G}$ of algebras over $\mathbb{C}$ endowed with a comultiplication $\Delta=\left\{\Delta_{\alpha, \beta}: H_{\alpha+\beta} \rightarrow H_{\alpha} \otimes H_{\beta}\right\}_{\alpha, \beta \in G}$, a counit $\epsilon: H_{0} \rightarrow \mathbb{C}$, and an antipode $S=\left\{S_{\alpha}: H_{\alpha} \rightarrow H_{-\alpha}\right\}_{\alpha \in G}$ which verify some compatibility conditions, see $[26,33,36]$.

Let $\mathcal{Z}^{0}=\operatorname{Span}\left\{K^{\ell a}: a \in \Lambda^{*}\right\}=\mathbb{C}\left[K_{1}^{ \pm \ell}, \ldots, K_{r}^{ \pm \ell}\right]$, recall $K_{i}=K^{a_{i}}$ where $\left\{a_{1}, \ldots, a_{r}\right\}$ is the fixed basis of $\Lambda^{*}$. Then $\mathcal{Z}^{0}$ is a Hopf subalgebra of $\mathcal{U}$ which is in the center of $\mathcal{U}$. Let $\operatorname{Hom}_{\mathrm{Alg}}\left(\mathcal{Z}^{0}, \mathbb{C}\right)$ be the group of characters on $\mathcal{Z}^{0}$ where the multiplication is given by $k h=(k \otimes h) \circ \Delta$ and $k^{-1}=\left.k \circ S\right|_{\mathcal{Z}^{0}}$. This group is isomorphic to $G=\left((\mathbb{C} / \mathbb{Z})^{r},+\right)$ : Indeed for each $\left(\alpha_{1}, \ldots, \alpha_{r}\right) \in \mathbb{C}^{r}$ denote its image in $(\mathbb{C} / \mathbb{Z})^{r}$ by $\bar{\alpha}=$ $\left(\bar{\alpha}_{1}, \cdots, \bar{\alpha}_{r}\right)$. Consider the isomorphism of groups $G \rightarrow \operatorname{Hom}_{\mathrm{Alg}}\left(\mathcal{Z}^{0}, \mathbb{C}\right)$ given by $\bar{\alpha}=\left(\bar{\alpha}_{1}, \cdots, \bar{\alpha}_{r}\right) \mapsto\left(K_{i}^{\ell} \mapsto \xi^{\ell \alpha_{i}}\right)$ for $i=1, \cdots, r$. We will use this isomorphism to identify $G$ with the characters on $\mathcal{Z}^{0}$.

We now give a Hopf $G$-coalgebra structure associated to $\mathcal{U}$. For each $\bar{\alpha}=\left(\bar{\alpha}_{1}, \cdots, \bar{\alpha}_{r}\right) \in G$, consider the ideal $I_{\bar{\alpha}}\left(\right.$ resp. $\left.I_{\bar{\alpha}}^{H}\right)$ of $\mathcal{U}\left(\right.$ resp. $\left.\mathcal{U}^{H}\right)$ generated by the elements $K_{i}^{\ell}-\xi^{\ell \alpha_{i}}$ for $i=1, \cdots, r$. Equivalently, $I_{\bar{\alpha}}$ and $I_{\bar{\alpha}}^{H}$ are the ideals generated by $(z-\bar{\alpha}(z))_{z \in \mathcal{Z}^{0}}$ where $\bar{\alpha}$ is a character under the identification $G \rightarrow \operatorname{Hom}_{\mathrm{Alg}}\left(\mathcal{Z}^{0}, \mathbb{C}\right)$.

\footnotetext{
${ }^{3}$ The original definition of Hopf group-coalgebra uses more general non abelian group.
} 
Let $\mathcal{U}_{\bar{\alpha}}=\mathcal{U} / I_{\bar{\alpha}}$ and $\mathcal{U}_{\bar{\alpha}}^{H}=\mathcal{U}^{H} / I_{\bar{\alpha}}^{H}$ with the projection maps

$$
\pi_{\bar{\alpha}}: \mathcal{U} \rightarrow \mathcal{U}_{\bar{\alpha}} \text { and } \pi_{\bar{\alpha}}^{H}: \mathcal{U}^{H} \rightarrow \mathcal{U}_{\bar{\alpha}}^{H} .
$$

As discussed in Example 2.3 of [18] there exists morphisms $\Delta_{\bar{\alpha}, \bar{\beta}}$ and $S_{\bar{\alpha}}$ given by the commutative diagrams:

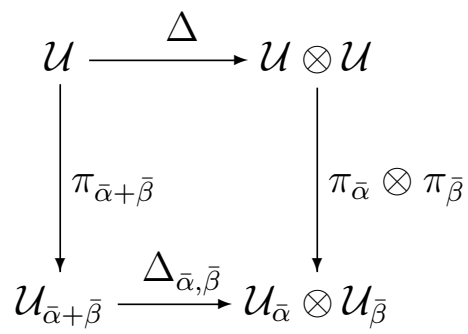

and

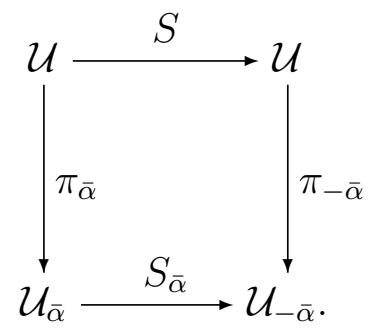

making $\mathcal{U}_{\bullet}=\left\{\mathcal{U}_{\bar{\alpha}}\right\}_{\bar{\alpha} \in G}$ a Hopf $G$-coalgebra. Similarly, using $\pi_{\bar{\alpha}}^{H}$ there exists $\Delta_{\bar{\alpha}, \bar{\beta}}^{H}$ and $S_{\bar{\alpha}}^{H}$ making $\left\{\mathcal{U}_{\bar{\alpha}}^{H}\right\}_{\bar{\alpha} \in G}$ a Hopf $G$-coalgebra. Remark that $\mathcal{U}_{\overline{0}}$ and its dual are ordinary Hopf algebras.

Let us now recall some definitions from [36, 18]

Definition 4.1. Let $H_{\bullet}=\left\{H_{\bar{\alpha}}\right\}_{\bar{\alpha} \in G}$ be a Hopf $G$-coalgebra.

(1) $H_{\bullet}$ is of finite type if for any $\bar{\alpha} \in G, \operatorname{dim}_{\mathbb{C}}\left(H_{\bar{\alpha}}\right)<\infty$.

(2) A G-grouplike element is a family $\left\{x_{\bar{\alpha}} \in H_{\bar{\alpha}}\right\}_{\bar{\alpha} \in G}$ such that $\Delta_{\bar{\alpha}, \bar{\beta}}\left(x_{\bar{\alpha}+\bar{\beta}}\right)=x_{\bar{\alpha}} \otimes x_{\bar{\beta}}$, for all $\bar{\alpha}, \bar{\beta} \in G$.

(3) A pivot for $H_{\bullet}$ is a $G$-grouplike element $\left\{g_{\bar{\alpha}}\right\}_{\bar{\alpha} \in G}$ such that $S_{-\bar{\alpha}} S_{\bar{\alpha}}=g_{\bar{\alpha}} \cdot g_{\bar{\alpha}}^{-1}$, for all $\bar{\alpha} \in G$. If $H_{\bullet}$ has a pivot we say it is a pivotal Hopf $G$-coalgebra.

(4) A right (resp. left) $G$-integral for $H_{\bullet}$ is a family of linear forms $\lambda^{R}=\left\{\lambda_{\bar{\alpha}}^{R} \in H_{\bar{\alpha}}^{*}\right\}_{\bar{\alpha} \in G}\left(\right.$ resp. $\left.\lambda^{L}=\left\{\lambda_{\bar{\alpha}}^{L} \in H_{\bar{\alpha}}^{*}\right\}_{\bar{\alpha} \in G}\right)$ such that

$$
\begin{gathered}
\left(\lambda_{\bar{\alpha}}^{R} \otimes \operatorname{Id}_{H_{\bar{\beta}}}\right) \Delta_{\bar{\alpha}, \bar{\beta}}(x)=\lambda_{\bar{\alpha}+\bar{\beta}}^{R}(x) 1_{\bar{\beta}}, \\
\left(\text { resp. }\left(\operatorname{Id}_{H_{\bar{\alpha}}} \otimes \lambda_{\bar{\beta}}^{L}\right) \Delta_{\bar{\alpha}, \bar{\beta}}(x)=\lambda_{\bar{\alpha}+\bar{\beta}}^{L}(x) 1_{\bar{\alpha}}\right)
\end{gathered}
$$

for all $\bar{\alpha}, \bar{\beta} \in G$ and $x \in H_{\bar{\alpha}+\bar{\beta}}$.

(5) $H_{\bullet}$ is unimodular if $H_{\overline{0}}$ is a unimodular Hopf algebra i.e. there exists a non zero element $\Upsilon \in H_{\overline{0}}$, called a two side cointegral, such that

$$
x \Upsilon=\Upsilon x=\varepsilon(x) \Upsilon \text { for all } x \in H_{\overline{0}} .
$$

From now we make the following assumption for $\mathcal{U}_{\overline{0}}$ :

Axiom 3. The Hopf $G$-coalgebra $\mathcal{U}_{\bullet}=\left\{\mathcal{U}_{\bar{\alpha}}, g_{\bar{\alpha}}\right\}_{\bar{\alpha} \in G}$ is unimodular.

Theorem 4.2 (Virelizier [36]). If $\left(H_{\bullet}, \Upsilon\right)$ is a finite type unimodular Hopf G-coalgebra, then

(1) the space of right $G$-integral is 1-dimensional,

(2) the right $G$-integral $\left\{\lambda_{\bar{\alpha}}^{R}\right\}_{\bar{\alpha} \in G}$ such that $\lambda_{\overline{0}}^{R}(\Upsilon)=1$ is unique,

(3) there exists a unique $G$-grouplike element $\gamma_{\bullet}$ called distinguished such that $\left\{\lambda_{\bar{\alpha}}^{R}\left(\gamma_{\bar{\alpha}} \cdot\right)\right\}_{\bar{\alpha} \in G}$ is a left $G$-integral,

(4) $\lambda_{\bar{\alpha}}^{R}\left(\gamma_{\bar{\alpha}} \cdot\right)=\lambda_{-\bar{\alpha}}^{R} \circ S_{\bar{\alpha}}$ and $\left(S_{-\bar{\alpha}} S_{\bar{\alpha}}\right)^{2}=\gamma_{\bar{\alpha}} \cdot \gamma_{\bar{\alpha}}^{-1}$ 
(5) $\lambda_{\bar{\alpha}}^{R}(x y)=\lambda_{\bar{\alpha}}^{R}\left(S_{-\bar{\alpha}} S_{\bar{\alpha}}(y) x\right)$ for all $x, y \in H_{\bar{\alpha}}$.

Proof. These results are adapted versions of [36], Theorem 3.6, Theorem 4.2 and Lemma 4.6 when the group $(G,+)$ is commutative and $H$. is unimodular so that the element called in [36] the distinguish element of $H_{\overline{0}}^{*}$ simplifies to the counit which acts trivially on $H_{\bar{\alpha}}$. Remark that the unimodularity is not needed for (1) and (3).

The Hopf $G$-coalgebras $\mathcal{U}_{\bullet}=\left\{\mathcal{U}_{\bar{\alpha}}\right\}_{\bar{\alpha} \in G}$ and $\mathcal{U}_{\bullet}^{H}=\left\{\mathcal{U}_{\bar{\alpha}}^{H}\right\}_{\bar{\alpha} \in G}$ are pivotal with the pivot given by the image $g_{\bar{\alpha}}$ of the pivotal element $g$ of $\mathcal{U}$ into $\mathcal{U}_{\bar{\alpha}}$ (resp. into $\mathcal{U}_{\bar{\alpha}}^{H}$ ). Moreover, $\mathcal{U}_{\bullet}$ is of finite type, thus it has a right $G$-integral.

4.2. Discrete Fourier transforms. In this subsection we recall the discrete Fourier transform from [17] that will be used to send the universal invariant into a Hopf $G$-coalgebra of finite type. The results allow us to construct an invariant of 3-manifolds in the next subsection.

Let $V$ be the space $\left(\mathfrak{h}^{*}\right)^{m}$ for some $m$. Denote $Z_{1}, \ldots, Z_{n}$ as the coordinate functions of $V=\left(\mathfrak{h}^{*}\right)^{m}$. Recall $\mathscr{C}^{\omega}\left(Z_{1}, \ldots, Z_{n}\right)$ is the set of entire functions on $V$ which is a subalgebra of $\left(\mathcal{U}^{H}\right)^{\widehat{\otimes} m}$. Given a map $f: \mathbb{C}^{n} \rightarrow \mathbb{C}$, we define $t_{i}(f)$ on $\mathbb{C}^{n}$ by

$$
t_{i}(f)\left(Z_{1}, \ldots, Z_{n}\right)=f\left(Z_{1}, \ldots, Z_{i}+1, \ldots, Z_{n}\right) \text { for } 1 \leq i \leq n .
$$

Let $\Lambda_{\vec{\alpha}}=\left\{\left(\alpha_{1}, \ldots, \alpha_{n}\right)+\mathbb{Z}^{n}\right\}$ be the lattice of $\mathbb{C}^{n}$ corresponding to $\vec{\alpha}=\left(\bar{\alpha}_{1}, \ldots, \bar{\alpha}_{n}\right) \in(\mathbb{C} / \mathbb{Z})^{n}$.

A function $f\left(Z_{1}, \ldots, Z_{n}\right) \in \mathscr{C}^{\omega}\left(Z_{1}, \ldots, Z_{n}\right)$ is called $\ell$-periodic in $Z_{i}$ on the lattice $\Lambda_{\vec{\alpha}}$ if it satisfies $f_{\left.\right|_{\vec{\alpha}}}=t_{i}^{\ell}(f)_{\left.\right|_{\vec{\alpha}}}$. The functions $\left\{\xi^{m Z_{i}}\right\}_{m \in \mathbb{Z}}^{i=1, \ldots, n}$ are $\ell$-periodic and $\xi^{\ell Z_{i}}-\xi^{\ell \alpha_{i}}$ is zero on $\Lambda_{\vec{\alpha}}$. Let $I_{\vec{\alpha}}$ be the ideal generated by $\xi^{\ell Z_{i}}-\xi^{\ell \alpha_{i}}$ for $1 \leq i \leq n$. For $m \leq n$, let $\mathscr{C}_{[m]}^{\omega}$ be the subring of entire functions that are Laurent polynomial in the first $m$ variables:

$$
\mathscr{C}_{[m]}^{\omega}=\mathscr{C}^{\omega}\left(Z_{m+1}, \ldots, Z_{n}\right)\left[\xi^{ \pm Z_{1}}, \ldots, \xi^{ \pm Z_{m}}\right] \subset \mathscr{C}^{\omega}\left(Z_{1}, \ldots, Z_{n}\right) .
$$

Then an element of $\mathscr{C}_{[m]}^{\omega} / I_{\vec{\alpha}}$ defines a $\ell$-periodic map in all first $m$ variables on $\Lambda_{\vec{\alpha}}$.

Proposition 4.3. Let $f=f\left(Z_{1}, \ldots, Z_{n}\right) \in \mathscr{C}^{\omega}\left(Z_{1}, \ldots, Z_{n}\right)$ be a $\ell$ periodic function on $\Lambda_{\vec{\alpha}}$ in the first $m$ variable. Then there is an element $\mathcal{F}_{\vec{\alpha}}(f) \in \mathscr{C}_{[m]}^{\omega}$ called the discrete Fourier transform of $f$ which is unique modulo $I_{\vec{\alpha}}$, coincides with $f$ on $\Lambda_{\bar{\alpha}_{1}, \ldots, \bar{\alpha}_{m}} \times \mathbb{C}^{n-m}$ and is given by

$$
\mathcal{F}_{\vec{\alpha}, m}(f)=\sum_{k_{1}, \ldots, k_{m}=0}^{\ell-1} a_{k_{1} \cdots k_{m}} \xi^{k_{1} Z_{1}+\cdots+k_{m} Z_{m}}
$$


The coefficients $a_{k_{1} \cdots k_{m}} \in \mathscr{C}^{\omega}\left(Z_{m+1}, \ldots, Z_{n}\right)$ (Fourier coefficients) are determined by

$$
a_{k_{1} \cdots k_{m}}=\frac{1}{\ell^{m}} \sum_{\substack{i_{1}, \ldots, i_{m}=0 \\ \beta_{j}=\alpha_{j}+i_{j}}}^{\ell-1} \xi^{-k_{1} \beta_{1}-\cdots-k_{m} \beta_{m}} f\left(\beta_{1}, \ldots, \beta_{m}, Z_{m+1}, \ldots, Z_{n}\right) .
$$

Proof. As proven, in [17, Proposition 4.6], for any fixed $\left(Z_{m+1}, \ldots, Z_{n}\right) \in$ $\mathbb{C}^{n-m}$, the element in Equation (26) is the unique linear combination of the elements $\left\{\xi^{k_{1} Z_{1}+\cdots+k_{m} Z_{m}}: 0 \leq k_{i}<\ell\right\}$ which coincide with $f$ for $\left(Z_{1}, \ldots, Z_{m}\right) \in \Lambda_{\bar{\alpha}_{1}, \ldots, \bar{\alpha}_{m}}$.

The complex analytic Nullstellensatz Theorem from the work of $\mathrm{H}$. Cartan [4] implies that $I_{\vec{\alpha}}$ is precisely the ideal of entire functions that vanish on $\Lambda_{\vec{\alpha}}$. Here we give an elementary proof that was shown to us by David E. Speyer:

Proposition 4.4. Let $f \in \mathscr{C}^{\omega}\left(Z_{1}, \ldots, Z_{n}\right)$ be zero on $\Lambda_{\bar{\alpha}_{1}, \ldots, \bar{\alpha}_{m}} \times \mathbb{C}^{n-m}$ then $f \in I_{\vec{\alpha}}$.

To prove this proposition, we need the following lemma:

Lemma 4.5. Let $\left\{\varphi_{k} \in \mathscr{C}^{\omega}\left(Z_{2}, \ldots, Z_{n}\right)\right\}_{k \in \mathbb{Z}}$ be a family of entire functions. Then there exists $\varphi \in \mathscr{C}^{\omega}\left(Z_{1}, \ldots, Z_{n}\right)$ such that

$$
\varphi_{k}=\varphi\left(k, Z_{2}, \ldots, Z_{n}\right)
$$

for all $k \in \mathbb{Z}$.

Proof. Let $\varphi\left(Z_{1}, \ldots, Z_{n}\right)$ be of the form

$$
\varphi_{0}(y)+\sum_{k=1}^{\infty} \frac{\prod_{m=1}^{k-1}\left(Z_{1}^{2}-m^{2}\right)}{\prod_{m=1}^{k-1}\left(k^{2}-m^{2}\right)}\left(a_{-k} \frac{k-Z_{1}}{2 k}+a_{k} \frac{k+Z_{1}}{2 k}\right)\left(\frac{Z_{1}^{2}}{k^{2}}\right)^{C_{k}}
$$

for some entire functions $a_{ \pm k} \in \mathscr{C}^{\omega}\left(Z_{2}, \ldots, Z_{n}\right)$ and some positive integers $C_{k}$ which we compute inductively as we will now describe. Note that, for $k>p$, the product $\prod_{m=1}^{k-1}$ vanishes at $Z_{1}= \pm p$, so terms beyond the $p$-th term don't affect the value of the sum at $Z_{1}= \pm p$. Also, at $Z_{1}= \pm k$, the $k^{\text {th }}$ term of the series reduces to $a_{ \pm k}$. So, if we have chosen $a_{m}, b_{m}$ and $C_{m}$ for $m<k$, there is a unique choice of $a_{ \pm k}$ which will make the sum correct at $Z_{1}= \pm k$. Make that choice for $a_{ \pm k}$. Then choose $C_{k}$ large enough that the $k^{\text {th }}$ summand has module less than $2^{-k}$ on the compact $\left\{\left|Z_{i}\right| \leq k-1, i=1 \cdots n\right\}$ for all $i=1 \cdots n$. (Since $Z_{1}^{2} / k^{2} \leq(1-1 / k)^{2}$ on this compact, taking $C_{k}$ large enough will work). Thus, the sum will be uniformly convergent on compact subsets, and will thus give an entire function.

Proof of Proposition 4.4. First remark that the entire function

$$
\left(\xi^{\ell Z}-\xi^{\ell \alpha}\right) \in \mathscr{C}^{\omega}(Z)
$$


only has simple zeros at integers. We prove the Proposition by induction on $n$ : for any $k \in \mathbb{Z}, f\left(\alpha_{1}+k, Z_{2}, \ldots, Z_{n}\right)$ is zero on the lattice $\Lambda_{\alpha_{2}, \ldots, \alpha_{n}}$. Then assume this function belongs to the ideal generated by $\left(\xi^{\ell Z_{i}}-\xi^{\ell \alpha_{i}}\right)_{i=2 \cdots n}$. Then we can write

$$
f\left(\alpha_{1}+k, Z_{2}, \ldots, Z_{n}\right)=\sum_{i=2}^{n}\left(\xi^{\ell Z_{i}}-\xi^{\ell \alpha_{i}}\right) \varphi_{i, k}\left(Z_{2}, \ldots, Z_{n}\right)
$$

then by the above Lemma applied to the family $\left(\varphi_{i, k}\right)_{k \in \mathbb{Z}}$, we obtain entire functions $\varphi_{i} \in \mathscr{C}^{\omega}\left(Z_{1}, \ldots, Z_{n}\right)$ such that for

$$
\varphi=\sum_{i=2}^{n}\left(\xi^{\ell Z_{i}}-\xi^{\ell \alpha_{i}}\right) \varphi_{i}
$$

$f-\varphi$ vanishes on $\left(\alpha_{1}+\mathbb{Z}\right) \times \mathbb{C}^{n-1}$. But then $\psi=\frac{f-\varphi}{\xi^{\ell Z_{1}}-\xi^{\ell \alpha_{1}}}$ is an entire function and $f=\varphi+\left(\xi^{\ell Z_{1}}-\xi^{\ell \alpha_{1}}\right) \psi \in I_{\vec{\alpha}}$.

The following proposition says, modulo the ideal $I_{\vec{\alpha}}$, the $\ell$-periodic elements are equal to their Fourier transform and power quadratic elements reduce to power linear. Here we use the notation $z_{\mathbf{0}}=$ $\left(Z_{1}, \ldots, Z_{n}\right)$ and say a linear element is any complex linear combination of the $Z_{i}$ while a quadratic element is a homogeneous degree 2 polynomial in the variables $z_{\bullet}$ with integer coefficients.

Proposition 4.6. If $f \in \mathscr{C}^{\omega}\left(Z_{1}, \ldots, Z_{n}\right)$ is periodic in its first $m$ variables then $f=\mathcal{F}_{\vec{\alpha}, m}(f)$ modulo $I_{\vec{\alpha}}$. Consequently, if $q\left(z_{\bullet}\right)$ is a quadratic element and $l$ is the linear element $l\left(z_{\bullet}\right)=\widetilde{q}\left(\tilde{\alpha}, z_{\bullet}\right)$ for any $\widetilde{\alpha}=\left(\alpha_{1}, \ldots, \alpha_{n}\right) \in \Lambda_{\vec{\alpha}}$, then $\xi^{q\left(z_{\bullet}\right)} \in \xi^{l\left(z_{\bullet}\right)} \mathbb{C}\left[\xi^{Z_{1}}, \ldots, \xi^{Z_{n}}\right]$ modulo $I_{\vec{\alpha}}$.

Proof. As $f-\mathcal{F}_{\vec{\alpha}, m}(f)$ is zero on $\Lambda_{\vec{\alpha}}$ then Proposition 4.4 implies it belongs to $I_{\vec{\alpha}}$. To prove the second statement of the proposition, let $e_{i}$ be the vector $(0, \ldots, 1, \ldots, 0)$ then $t_{i}^{\ell}\left(l\left(z_{\bullet}\right)\right)-l\left(z_{\bullet}\right)=\widetilde{q}\left(\widetilde{\alpha}, \ell e_{i}\right)=$ $\ell \widetilde{q}\left(e_{i}, \widetilde{\alpha}\right)$ while $t_{i}^{\ell}\left(q\left(z_{\bullet}\right)\right)-q\left(z_{\bullet}\right)=\widetilde{q}\left(\ell e_{i}, z_{\bullet}\right)+q\left(\ell e_{i}\right)$ which on $\Lambda_{\vec{\alpha}}$ takes values in $\ell \widetilde{q}\left(e_{i}, \widetilde{\alpha}\right)+\ell \mathbb{Z}$. So $\xi^{q\left(z_{\bullet}\right)-l\left(z_{\bullet}\right)}$ is $\ell$-periodic in all its variable on $\Lambda_{\vec{\alpha}}$ so, modulo $I_{\vec{\alpha}}$, it is equal to $\mathcal{F}_{\vec{\alpha}, n}\left(\xi^{q\left(z_{\bullet}\right)-l\left(z_{\bullet}\right)}\right)$ and $\xi^{q\left(z_{\bullet}\right)}=$ $\xi^{l\left(z_{\bullet}\right)} \mathcal{F}_{\vec{\alpha}, n}\left(\xi^{q\left(z_{\bullet}\right)-l\left(z_{\bullet}\right)}\right)$.

Next we would like to apply the above results to the context of the topological unrolled quantum groups. For $\vec{\alpha}=\left(\bar{\alpha}_{1}, \ldots, \bar{\alpha}_{n}\right) \in G^{n}$ we denote by $I_{\vec{\alpha}}^{H}$ the ideal of $\left(\mathcal{U}^{H}\right)^{\widehat{\otimes} n}$ generated by the $r n$ elements $\xi^{\ell H_{i, j}}-\xi^{\ell \bar{\alpha}_{i, j}}$ where $\bar{\alpha}_{i, j}=\bar{\alpha}_{j}\left(H_{i}\right) \in \mathbb{C} / \mathbb{Z}$ is the value of $H_{i, j}$ at $\vec{\alpha}$. Recall in Subsection 3.4 we define the power linear $\mathcal{L}_{n}$ and quadratic elements $\mathcal{Q}_{n}$. In particular, recall $\mathcal{L}_{n}=\left\{\xi^{H}: H \in \mathfrak{H}^{(n)}\right\}$ where the elements $\left\{H_{i, j}\right\}_{i=1, \ldots, r, j=1, \ldots, n}$ are a basis of $\mathfrak{H}^{(n)}$. Let $\mathcal{L} \mathcal{U}_{\bar{\alpha}}$ be the image of $\mathcal{L}_{1} \mathcal{U}$ in the quotient $\mathcal{U}_{\bar{\alpha}}^{H}$. 
Proposition 4.7. Let $\vec{\alpha}=\left(\bar{\alpha}_{1}, \ldots, \bar{\alpha}_{m}, \ldots, \bar{\alpha}_{m+n}\right) \in G^{m+n}$ and let $h_{\bullet}$ denotes the set of $r(m+n)$ variables $\left\{H_{i, j}\right\}_{i=1, \ldots, r, j=1, \ldots, m+n}$. Let

$$
u \in \mathcal{Q}_{m+n} \mathcal{L}_{m+n} \mathcal{U}^{\otimes m+n} \subset \mathscr{C}^{\omega}\left(h_{\bullet}\right) \otimes W^{\otimes m+n} \simeq\left(\mathcal{U}^{H}\right)^{\widehat{\otimes} m+n}
$$

be an $\ell$-periodic element in the first rm variables $\left\{H_{i, j}\right\}_{i=1, \ldots, r, j=1, \ldots, m}$ on the lattice $\Lambda_{\vec{\alpha}}$. Then

$$
\pi(u) \in \mathcal{U}_{\bar{\alpha}_{1}} \otimes \cdots \otimes \mathcal{U}_{\bar{\alpha}_{m}} \otimes \mathcal{L} \mathcal{U}_{\bar{\alpha}_{m+1}} \otimes \cdots \otimes \mathcal{L U}_{\bar{\alpha}_{m+n}}
$$

where $\pi:\left(\mathcal{U}^{H}\right)^{\widehat{\otimes} m+n} \rightarrow\left(\mathcal{U}^{H}\right)^{\widehat{\otimes} m+n} / I_{\vec{\alpha}}^{H}$ is the projection.

Proof. Let us write $u=\sum_{k} u_{k}\left(h_{\bullet}\right) w_{k}$ in a basis $\left(w_{k}\right)_{k}$ of $W^{\otimes m+n}$. By the previous proposition, each $u_{k}$ is equal modulo $I_{\vec{\alpha}}^{H}$ to a Laurent polynomial in $\mathbb{C}\left[\xi^{ \pm H_{i, j}}\right]$ times a power linear element in the last $r n$ variables $\xi^{\sum \beta_{i, j} H_{i, j}}$ where the sum range for $i=1 \cdots r, j=m+1 \cdots m+n$. Then for $\gamma_{j}\left(H_{1}, \ldots, H_{r}\right)=\sum_{i=1}^{r} \beta_{i, j} H_{i}$, we have:

$$
\pi(u) \in \mathcal{U}_{\bar{\alpha}_{1}} \otimes \cdots \otimes \mathcal{U}_{\bar{\alpha}_{m}} \otimes \xi^{\gamma_{m+1}} \mathcal{U}_{\bar{\alpha}_{m+1}} \otimes \cdots \otimes \xi^{\gamma_{m+n}} \mathcal{U}_{\bar{\alpha}_{m+n}} .
$$

4.3. The symmetrized $G$-integral. Recall that we denote by $h=$ $\left(H_{1}, \ldots, H_{r}\right)$ and $h_{j}=\left(H_{1, j}, \ldots, H_{r, j}\right)$ so for example, $\mathcal{H}=\xi^{\mathrm{Q}\left(h_{1}, h_{2}\right)}$. Fix $\alpha \in \mathfrak{H}$ and let $\bar{\alpha} \in G$ be its class modulo $\Lambda$. Then we have

$$
\begin{aligned}
\widetilde{\mathrm{Q}}\left((-\alpha, \alpha),\left(h_{1}, h_{2}\right)\right) & =\mathrm{Q}\left(h_{1}-\alpha, h_{2}+\alpha\right)-\mathrm{Q}\left(h_{1}, h_{2}\right)+\mathrm{Q}(\alpha, \alpha) \\
& =\mathrm{Q}\left(\alpha, h_{1}\right)-\mathrm{Q}\left(\alpha, h_{2}\right) .
\end{aligned}
$$

So $\xi^{\widetilde{Q}\left((-\alpha, \alpha),\left(h_{1}, h_{2}\right)\right)}=L\left(h_{1}-h_{2}\right)$ where $L(h)=\xi^{\mathrm{Q}(\alpha, h)}$ and Proposition 4.6 implies that $L\left(h_{2}-h_{1}\right) \mathcal{H}$ is in $\mathbb{C}\left[\xi^{h_{1}}, \xi^{h_{2}}\right]$ modulo $I_{(-\alpha, \alpha)}$. In particular, $L\left(h_{2}-h_{1}\right) \mathcal{H}$ is $\ell$-periodic in all its variable on $\Lambda_{(-\bar{\alpha}, \bar{\alpha})}$. Let $\mathcal{H}_{\alpha} \in \mathcal{U}_{-\bar{\alpha}} \otimes \mathcal{U}_{\bar{\alpha}}$ be its Fourier transform on this lattice. Hence $\mathcal{H}=L_{\alpha} \mathcal{H}_{\alpha}$ modulo $I_{(-\alpha, \alpha)}$ where $L_{\alpha}=L\left(h_{1}-h_{2}\right)$.

Proposition 4.8. The finite dimensional Hopf algebra $\mathcal{U}_{\overline{0}}$ is ribbon with universal $R$-matrix

$$
\mathcal{R}_{\overline{0}}=\mathcal{H}_{0} \check{\mathcal{R}} \in \mathcal{U}_{\overline{0}} \otimes \mathcal{U}_{\overline{0}}
$$

and twist $\theta_{\overline{0}}=g^{-1}\left(m \circ\left(S^{2} \otimes \mathrm{Id}\right)\left(\mathcal{R}_{\overline{0}}\right)\right)=m\left(\mathcal{H}_{0}\right) \sum_{i} y_{i} g x_{i} B_{\left|x_{i}\right|} \in \mathcal{U}_{\overline{0}}$.

Proof. For $\alpha=0$, one has $L_{\alpha}=1$ and the image of the $R$-matrix in $\mathcal{U}_{\overline{0}}^{H} \widehat{\otimes} \mathcal{U}_{\overline{0}}^{H}$ belongs in fact to $\mathcal{U}_{\overline{0}} \otimes \mathcal{U}_{\overline{0}}$. So $\mathcal{R}=\mathcal{H}_{0} \mathcal{R}$ modulo the ideal $I_{\overrightarrow{0}}^{H}$ and $\mathcal{R}_{\overline{0}}$ inherits all properties of the $R$-matrix $\mathcal{R}$. Finally the formula for the twist comes from $\theta=g u^{-1}$ and Equation (22).

Both the quotient morphism $\mathcal{U}^{H} \rightarrow \mathcal{U}_{\overline{0}}^{H}$ and the inclusion morphism $\mathcal{U}_{\overline{0}} \rightarrow \mathcal{U}_{\overline{0}}^{H}$ are morphisms of ribbon Hopf algebra.

To build a invariant of 3-manifold, we will need the following axiom that will fix the normalization of the cointegral $\Upsilon$ and integral $\lambda^{R}$ :

Axiom 4. Let $\delta=\lambda_{\overline{0}}^{R}\left(\theta_{\overline{0}}\right)$ and $\bar{\delta}=\lambda_{\overline{0}}^{R}\left(\theta_{\overline{0}}^{-1}\right)$. We assume: $\delta \bar{\delta}=1$.

The true assumption in this axiom is the "non-degeneracy" condition $\delta \bar{\delta} \neq 0$. Indeed, we have 
Proposition 4.9. If $\delta \bar{\delta} \neq 0$ then there exists a choice of $\Upsilon$ so that Axiom 4 is satisfied.

Proof. If $\delta \bar{\delta} \neq 0$, it has a square root $\rho \in \mathbb{C}$ such that replacing $\Upsilon$ with $\rho \Upsilon$ will lead to a new right integral equal to $\frac{1}{\rho} \lambda^{R}$ for which Axiom 4 is satisfied.

For $\bar{\alpha} \in G$ and $\nu \in \Lambda$, let $\mathrm{p}_{\nu}^{\bar{\alpha}}: \mathcal{U}_{\bar{\alpha}} \rightarrow \mathcal{U}_{\bar{\alpha}}$ be the projection on the subspace of weight $\nu$ of $\mathcal{U}_{\bar{\alpha}}$.

Lemma 4.10. The cointegral $\Upsilon \in \mathcal{U}_{\overline{0}}$ and the right $G$-integral $\left\{\lambda_{\bar{\alpha}}^{R}\right\}_{\bar{\alpha} \in G}$ are of degree 0 , meaning they satisfy: $\Upsilon=\mathrm{p}_{0}^{\overline{0}}(\Upsilon)$ and

$$
\lambda_{\bar{\alpha}}^{R}=\lambda_{\bar{\alpha}}^{R} \circ \mathrm{p}_{0}^{\bar{\alpha}}
$$

for all $\bar{\alpha} \in G$.

Proof. For any $\nu \in \Lambda$, let $\Upsilon_{\nu}=\mathrm{p}_{\nu}^{\overline{0}}(\Upsilon)$. Now for any homogeneous $x \in \mathcal{U}_{\overline{0}}$ of weight $|x|$,

$$
x \Upsilon_{\nu}=\mathrm{p}_{|x|+\nu}^{\overline{0}}(x \Upsilon)=\varepsilon(x) \mathrm{p}_{|x|+\nu}^{\overline{0}}(\Upsilon)=\varepsilon(x) \Upsilon_{\nu},
$$

where the last equality is because $\varepsilon(x)=0$ unless $|x|=0$. Hence $\Upsilon_{\nu}$ is a left cointegral in $\mathcal{U}_{\overline{0}}$. Since the space on left cointegral is one dimensional (see [29, Theorem 10.2.2]), we get that $\Upsilon_{\nu}=\Upsilon$ or $\Upsilon_{\nu}=0$. Finally, as $\Upsilon=\sum_{\nu} \Upsilon_{\nu}$, there exists a unique $\nu_{0} \in \Lambda$ such that $\Upsilon=\Upsilon_{\nu_{0}}$.

Similarly, for any $\bar{\alpha} \in G$ and for any $\nu \in \Lambda$, let $\lambda_{\bar{\alpha}}^{\nu}=\lambda_{\bar{\alpha}}^{R} \circ \mathrm{p}_{\nu}^{\bar{\alpha}}$. Then let $x \in \mathcal{U}_{\bar{\alpha}+\bar{\beta}}$ of weight $|x|$. Then we have $\lambda_{\bar{\alpha}}^{\nu}\left(x_{(1)}\right) x_{(2)}=\lambda_{\bar{\alpha}}^{R}\left(\mathrm{p}_{\nu}^{\bar{\alpha}}\left(x_{(1)}\right)\right) x_{(2)}=$ $\lambda_{\bar{\alpha}}^{R}\left(x_{(1)}\right) \mathrm{p}_{|x|-\nu}^{\bar{\beta}}\left(x_{(2)}\right)=\lambda_{\bar{\alpha}+\bar{\beta}}^{R}(x) \mathrm{p}_{|x|-\nu}^{\bar{\beta}}\left(1_{\mathcal{U}_{\bar{\beta}}}\right)=\lambda_{\bar{\alpha}+\bar{\beta}}^{\nu}(x) 1_{\mathcal{U}_{\bar{\beta}}}$. Thus $\lambda^{\nu}$ is a right $G$-integral. Once again, since the space of right $G$-integral is one dimensional, one has $\lambda^{\nu}=\lambda^{R}$ or $\lambda^{\nu}=0$ and since $\lambda_{\overline{0}}^{R}(\Upsilon)=1$, we have $\lambda^{R}=\lambda^{\nu_{0}}$.

The fact that $\nu_{0}=0$ is now the consequence of Axiom 4 since $\theta_{\overline{0}}$ has weight 0 and $\lambda^{R}\left(\theta_{\overline{0}}\right) \neq 0$.

The next property of $\mathcal{U}_{\bullet}$ is called unibalanced in $[3,18]$ :

Proposition 4.11. The distinguished element of $\mathcal{U}_{\bullet}$ is the square of its pivot:

$$
\gamma_{\bar{\alpha}}=g_{\bar{\alpha}}^{2} \text { for all } \bar{\alpha} \in G \text {. }
$$

To prove the proposition, we use the following Lemma (see also [36]):

Lemma 4.12. Let $\Upsilon_{(1)}^{-\bar{\alpha}} \otimes \Upsilon_{(2)}^{\bar{\alpha}}=\Delta_{-\bar{\alpha}, \bar{\alpha}}(\Upsilon) \in \mathcal{U}_{-\bar{\alpha}} \otimes \mathcal{U}_{\bar{\alpha}}$. Then

$$
\lambda_{-\bar{\alpha}}^{R}\left(\Upsilon_{(1)}^{-\bar{\alpha}}\right) \Upsilon_{(2)}^{\bar{\alpha}}=1 \text { and } \lambda_{-\bar{\alpha}}^{R}\left(\Upsilon_{(2)}^{-\bar{\alpha}}\right) \Upsilon_{(1)}^{\bar{\alpha}}=\gamma_{\bar{\alpha}}
$$

Furthermore for any $x \in \mathcal{U}_{-\bar{\alpha}}$, one has

$x \Upsilon_{(1)}^{-\bar{\alpha}} \otimes \Upsilon_{(2)}^{\bar{\alpha}}=\Upsilon_{(1)}^{-\bar{\alpha}} \otimes S_{-\bar{\alpha}}^{-1}(x) \Upsilon_{(2)}^{\bar{\alpha}}$ and $\Upsilon_{(1)}^{-\bar{\alpha}} x \otimes \Upsilon_{(2)}^{\bar{\alpha}}=\Upsilon_{(1)}^{-\bar{\alpha}} \otimes \Upsilon_{(2)}^{\bar{\alpha}} S_{-\bar{\alpha}}(x)$. 
Proof. First $\left(\lambda^{R} \otimes \mathrm{Id}\right)\left(\Delta_{-\bar{\alpha}, \bar{\alpha}}(\Upsilon)\right)=\lambda^{R}(\Upsilon) 1=1$ and since $\lambda^{L}=\lambda^{R}(\gamma \cdot)$ is a left $G$-integral, $\left(\operatorname{Id} \otimes \lambda^{R}\right)\left(\Delta_{-\bar{\alpha}, \bar{\alpha}}(\Upsilon)\right)=\left(\gamma \otimes \lambda^{L}\right)\left(\Delta_{-\bar{\alpha}, \bar{\alpha}}\left(\gamma^{-1} \Upsilon\right)\right)=\gamma$.

$$
\begin{gathered}
\Upsilon_{(1)}^{-\bar{\alpha}} x \otimes \Upsilon_{(2)}^{\bar{\alpha}}=\Upsilon_{(1)}^{-\bar{\alpha}} x_{(1)} \otimes \Upsilon_{(2)}^{\bar{\alpha}} x_{(2)} S_{-\bar{\alpha}}\left(x_{(3)}\right)= \\
\Delta_{-\bar{\alpha}, \bar{\alpha}}\left(\Upsilon x_{(1)}\right) 1 \otimes S_{-\bar{\alpha}}\left(x_{(2)}\right)=\Upsilon_{(1)}^{-\bar{\alpha}} \otimes \Upsilon_{(2)}^{\bar{\alpha}} S_{-\bar{\alpha}}(x) .
\end{gathered}
$$

Similarly,

$$
\begin{aligned}
& x \Upsilon_{(1)}^{-\bar{\alpha}} \otimes \Upsilon_{(2)}^{\bar{\alpha}}=x_{(1)} \Upsilon_{(1)}^{-\bar{\alpha}} \otimes S_{-\bar{\alpha}}^{-1}\left(x_{(3)}\right) x_{(2)} \Upsilon_{(2)}^{\bar{\alpha}}= \\
& 1 \otimes S_{-\bar{\alpha}}^{-1}\left(x_{(2)}\right) \Delta_{-\bar{\alpha}, \bar{\alpha}}\left(x_{(1)} \Upsilon\right)=\Upsilon_{(1)}^{-\bar{\alpha}} \otimes S_{-\bar{\alpha}}^{-1}(x) \Upsilon_{(2)}^{\bar{\alpha}}
\end{aligned}
$$

Proof of Proposition 4.11. We have

$$
\gamma_{\bar{\alpha}}=\lambda_{-\bar{\alpha}}^{R}\left(\Upsilon_{(2)}^{-\bar{\alpha}}\right) \Upsilon_{(1)}^{\bar{\alpha}}=\left(\lambda_{-\bar{\alpha}}^{R} \otimes \operatorname{Id}\right)\left(\mathcal{R} \Delta_{-\bar{\alpha}, \bar{\alpha}}(\Upsilon) \mathcal{R}^{-1}\right)
$$

and

$$
\mathcal{R} \Delta_{-\bar{\alpha}, \bar{\alpha}}(\Upsilon) \mathcal{R}^{-1}=L_{\alpha} \mathcal{H}_{\alpha} \check{\mathcal{R}} \Delta_{-\bar{\alpha}, \bar{\alpha}}(\Upsilon)\left(L_{\alpha} \mathcal{H}_{\alpha} \check{\mathcal{R}}\right)^{-1}
$$

Now we write the linear element $L_{\alpha}$ given at the beginning of this subsection as $L_{\alpha}=L(h) \otimes L(-h)=L\left(h_{1}\right) L\left(h_{2}\right)^{-1}$ where $L(h)=$ $\xi^{\mathrm{Q}(\alpha, h)} \in \mathcal{L}_{1}$. Consider the application $f_{+}: \mathcal{U}_{-\bar{\alpha}}^{H} \otimes \mathcal{U}_{\bar{\alpha}}^{H} \rightarrow \mathcal{U}_{\bar{\alpha}}^{H}$ given by $f_{+}(x \otimes y)=S(x) y$ and $f_{-}: \mathcal{U}_{-\bar{\alpha}}^{H} \otimes \mathcal{U}_{\bar{\alpha}}^{H} \rightarrow \mathcal{U}_{\bar{\alpha}}^{H}$ given by $f_{-}(x \otimes y)=$ $y S^{-1}(x)$. Remark that $f_{-}(\mathcal{R})=f_{-}((S \otimes S)(\mathcal{R}))=u=g \theta^{-1}$ and $f_{+}\left(\mathcal{R}^{-1}\right)=f_{+}((S \otimes \mathrm{Id})(\mathcal{R}))=S\left(u^{-1}\right)=g \theta$.

Then from Lemma above, one has:

$$
\begin{gathered}
\mathcal{R}_{-\bar{\alpha}, \bar{\alpha}}(\Upsilon) \mathcal{R}^{-1}=L_{\alpha}\left(1 \otimes f_{-}\left(\mathcal{H}_{\alpha} \check{\mathcal{R}}\right)\right) \Delta_{-\bar{\alpha}, \bar{\alpha}}(\Upsilon)\left(1 \otimes f_{+}\left(\check{\mathcal{R}}^{-1} \mathcal{H}_{\alpha}^{-1}\right)\right) L_{\alpha}^{-1} \\
=L_{\alpha}\left(1 \otimes f_{-}\left(L_{\alpha}^{-1} \mathcal{R}\right)\right) \Delta_{-\bar{\alpha}, \bar{\alpha}}(\Upsilon)\left(1 \otimes f_{+}\left(\mathcal{R}^{-1} L_{\alpha}\right)\right) L_{\alpha}^{-1} \\
=L_{\alpha} L\left(h_{2}\right)\left(1 \otimes f_{-}(\mathcal{R})\right) L\left(h_{2}\right) \Delta_{-\bar{\alpha}, \bar{\alpha}}(\Upsilon) L\left(-h_{2}\right)\left(1 \otimes f_{+}\left(\mathcal{R}^{-1}\right)\right) L\left(-h_{2}\right) L_{\alpha}^{-1} \\
=\left(L(h) \otimes g \theta^{-1} L(h)\right) \Delta_{-\bar{\alpha}, \bar{\alpha}}(\Upsilon)(L(-h) \otimes L(-h) g \theta) .
\end{gathered}
$$

Finally, since $\lambda^{R}$ is homogeneous of weight 0 , only terms with $\Upsilon_{(1)}$ commuting with $L\left( \pm h_{1}\right)$ will contribute so:

$$
\begin{gathered}
\lambda_{-\bar{\alpha}}^{R} \otimes \operatorname{Id}\left(\mathcal{R} \Delta_{-\bar{\alpha}, \bar{\alpha}}(\Upsilon) \mathcal{R}^{-1}\right)=\lambda_{-\bar{\alpha}}^{R} \otimes \operatorname{Id}\left(\left(1 \otimes g \theta^{-1} L(h)\right) \Delta_{-\bar{\alpha}, \bar{\alpha}}(\Upsilon)(1 \otimes L(-h) g \theta)\right) \\
=f_{-}(\mathcal{R}) L\left(h_{2}\right) L\left(-h_{2}\right) f_{+}\left(\mathcal{R}^{-1}\right)=u S\left(u^{-1}\right)=g^{2} .
\end{gathered}
$$

The following terminology first appeared in [3] in the non-graded case, then in [18] for the example of special linear superalgebra $\mathfrak{s l}(2 \mid 1)$.

Definition 4.13. The symmetrised $G$-integral of $H_{\text {• }}$ is the family of linear forms $\mu=\left\{\mu_{\bar{\alpha}} \in \mathcal{U}_{\bar{\alpha}}^{*}\right\}_{\bar{\alpha} \in G}$ defined by

$$
\mu_{\bar{\alpha}}(x)=\lambda_{\bar{\alpha}}\left(g_{\bar{\alpha}} x\right) \text { for } x \in \mathcal{U}_{\bar{\alpha}} .
$$

The symmetrized $G$-integral $\mu$ has the properties of a $G$-trace in [36]. More precisely, we have the properties below: 
Proposition 4.14. These equalities hold

$$
\begin{aligned}
\left(\mu_{\bar{\alpha}} \otimes g_{\bar{\beta}}\right) \Delta_{\bar{\alpha}, \bar{\beta}}(x) & =\mu_{\bar{\alpha}+\bar{\beta}}(x) 1_{\bar{\beta}} \text { for } x \in \mathcal{U}_{\bar{\alpha}+\bar{\beta}}, \\
\left(g_{\bar{\alpha}}^{-1} \otimes \mu_{\bar{\beta}}\right) \Delta_{\bar{\alpha}, \bar{\beta}}(x) & =\mu_{\bar{\alpha}+\bar{\beta}}(x) 1_{\bar{\beta}} \text { for } x \in \mathcal{U}_{\bar{\alpha}+\bar{\beta}}, \\
\mu_{\bar{\alpha}}(x y) & =\mu_{\bar{\alpha}}(y x) \text { for } x, y \in \mathcal{U}_{\bar{\alpha}}, \\
\mu_{-\bar{\alpha}}\left(S_{ \pm \bar{\alpha}}^{ \pm 1}(x)\right) & =\mu_{\bar{\alpha}}(x) \text { for } x \in \mathcal{U}_{\bar{\alpha}} . \\
\mu_{\bar{\alpha}}(x) & =\mu_{\bar{\alpha}}\left(\mathrm{p}_{0}^{\bar{\alpha}}(x)\right) \text { for } x \in \mathcal{U}_{\bar{\alpha}} .
\end{aligned}
$$

Proof. These identities are directly obtained from Theorem 4.2 and Lemma 4.10 by the change of variable $x \mapsto g_{\bar{\alpha}+\bar{\beta}} x$ and by using the properties of the pivot.

The following proposition implies Examples 2.3 and 2.4 satisfy Axiom 3:

Proposition 4.15. Suppose that $\Lambda^{\prime}$ is a rank $r$ sub-lattice of $\Lambda^{*}$ as in Remark 2.2 and let $\mathcal{U}_{\overline{0}}^{\prime}=\mathcal{U}_{\Lambda^{\prime}} /\left(I_{\overline{0}} \cap \mathcal{U}_{\Lambda^{\prime}}\right)$. If $\mathcal{U}_{\overline{0}}^{\prime}$ is unimodular with a weight 0 cointegral, then so is $\mathcal{U}_{\overline{0}}$.

Proof. Since $\Lambda^{\prime}$ has rank $r$, the quotient $\Lambda / \Lambda^{\prime}$ is finite with $d$ elements. Let $\left(k_{1}, \ldots, k_{d}\right) \in \Lambda^{d}$ be representatives of these elements, then $\mathcal{U}_{\overline{0}}^{\prime} \subset \mathcal{U}_{\overline{0}}$ and as $\mathbb{C}$-vector space, $\mathcal{U}_{\overline{0}}=\bigoplus_{i} k_{i} \mathcal{U}_{\overline{0}}^{\prime}$. Let $k_{\Upsilon}=\sum_{i} k_{i}$. If $\Upsilon^{\prime}$ is the cointegral of $\mathcal{U}_{\overline{0}}^{\prime}$ then one easily check that $\Upsilon=k_{\Upsilon} \Upsilon^{\prime}=\Upsilon^{\prime} k_{\Upsilon}$ is a two side cointegral for $\mathcal{U}_{\overline{0}}$. In particular, if $k \in \Lambda$, then $k$ will permute elements of $\Lambda / \Lambda^{\prime}$ and there exists $K_{i}^{\prime} \in \Lambda^{\prime}$ such that $k k_{\Upsilon}=\sum_{i} k_{i} K_{i}^{\prime}$ thus $\Upsilon k=k \Upsilon=\sum_{i} k_{i} \varepsilon\left(K_{i}^{\prime}\right) \Upsilon^{\prime}=\varepsilon(k) \Upsilon$.

Since the $\Lambda^{\prime}$ version of the quantum groups considered in Examples 2.3 and 2.4 are unimodular (see [9]), we have:

Corollary 4.16. The quantum groups $\mathcal{U}_{\overline{0}}$ associated to Examples 2.3 and 2.4 are unimodular. Thus, the quantum groups $\mathcal{U}$ of Examples 2.3 and 2.4 satisfy Axiom 3.

Example 4.17. Here we build upon Examples 2.3 (which is continued in Examples 2.7 and 4.22). As shown in [18, section 5.1], the symmetrized integral does not depend on $\bar{\alpha}$ in the PBW basis: There exists $\eta \in \mathbb{C}$ such that it is given for any $0 \leq i, j \leq \ell^{\prime}-1$ and any $0 \leq k \leq \ell-1$ by

$$
\mu_{\bar{\alpha}}\left(E^{i} F^{j} K^{k}\right)=\eta \delta_{i, \ell^{\prime}-1} \delta_{j, \ell^{\prime}-1} \delta_{k, 0} .
$$

Furthermore, Equation (30) and the commutation relations for $K$ imply that the same formula holds for the product of $E^{i}, F^{j}$ and $K^{k}$ in any order. Using the Fourier transform, it can be rewritten for any $\ell$ periodic element $x=\sum_{i, j} E^{i} F^{j} \varphi_{i j}(H)$ as

$$
\mu_{\bar{\alpha}}(x)=\frac{\eta}{\ell} \sum_{k=0}^{\ell-1} \varphi_{\ell^{\prime}-1, \ell^{\prime}-1}(\alpha+k) \text {. }
$$


We will apply this to compute $\lambda_{\overline{0}}\left(\theta_{\overline{0}}^{ \pm 1}\right)$ : In the formula of $\check{\mathcal{R}}$, only the last term will contribute and this term is $c E^{\ell^{\prime}-1} \otimes F^{\ell^{\prime}-1}$ where

$$
c=\frac{\left(\xi-\xi^{-1}\right)^{\ell^{\prime}-1}}{\left[\ell^{\prime}-1 ; \xi^{-2}\right] !}=\frac{-(-1)^{\ell} \xi}{\ell^{\prime}}\left(\xi-\xi^{-1}\right)^{2\left(\ell^{\prime}-1\right)} .
$$

Thus

$$
\begin{aligned}
\lambda_{\overline{0}}\left(\theta_{\overline{0}}\right)=\mu_{\overline{0}}\left(g^{-2} S(u)^{-1}\right) & =\mu_{\overline{0}}\left(c K^{-2} \xi^{2 H^{2}} E^{\ell^{\prime}-1} K^{2 \ell^{\prime}-2} F^{\ell^{\prime}-1} K^{2-2 \ell^{\prime}}\right) \\
= & c \xi^{-2} \frac{\eta}{\ell} \sum_{k=0}^{\ell-1} \xi^{2 k^{2}-2 k} .
\end{aligned}
$$

This last Gauss sum is 0 if and only if $\ell \in 8 \mathbb{N}$ (see for example [6]). Similarly, using that $u=\sum_{i} S^{2}\left(b_{i}\right) S\left(a_{i}\right)=m\left(\mathcal{H}^{-1}\right) \sum_{i} S^{2}\left(y_{i}\right) S\left(x_{i}\right)$, one has

$$
\begin{aligned}
& \lambda_{\overline{0}}\left(\theta_{\overline{0}}^{-1}\right)=\mu_{\overline{0}}\left(g^{-2} u\right)=\mu_{\overline{0}}\left(c g^{-2} m\left(\mathcal{H}^{-1}\right) S^{2}\left(F^{\ell^{\prime}-1}\right) S\left(E^{\ell^{\prime}-1}\right)\right) \\
= & \mu_{\overline{0}}\left(c g^{-2} \xi^{-2 H^{2}} \xi^{2-2 \ell^{\prime}} F^{\ell^{\prime}-1} \xi^{-2} E^{\ell^{\prime}-1} K^{2-2 \ell^{\prime}}\right)=c \frac{\eta}{\ell} \sum_{k=0}^{\ell-1} \xi^{2 k^{2}-2 k} .
\end{aligned}
$$

Thus if $\ell \notin 8 \mathbb{N}$, one can choose $\eta=\frac{\xi \ell}{c}\left(\sum_{k=0}^{\ell-1} \xi^{2 k^{2}-2 k}\right)^{-1}$ and Axiom 4 holds with $\lambda_{\overline{0}}\left(\theta_{\overline{0}}^{-1}\right)=\xi=\lambda_{\overline{0}}\left(\theta_{\overline{0}}\right)^{-1}$.

Example 4.18. Here we build upon Example 2.4 (which is continued in Examples 2.8 and 4.22) and sketch a proof that Axiom 4 hold for these examples. To simplify notation in this example we set $\mathcal{U}^{\prime}=\mathcal{U}_{\Lambda^{\prime}}$. First, the restriction of right integral of $\mathcal{U}_{\overline{0}}$ to $\mathcal{U}_{\overline{0}}^{\prime}=\mathcal{U}^{\prime} /\left(I_{\overline{0}} \cap \mathcal{U}^{\prime}\right)$ is a right integral for $\mathcal{U}_{\overline{0}}^{\prime}$. Second, $R_{\overline{0}}$ and $\theta_{\overline{0}}$ belongs to the subalgebra $\mathcal{U}_{\overline{0}}^{\prime}$. Hence $\delta$ can be computed in the small quantum group $\mathcal{U}_{\overline{0}}^{\prime}$. Finally, in $[24,25]$ it is shown that $\mathcal{U}_{0}^{\prime}$ is ribbon and factorizable thus [10, Corollary 3.9] implies it is non degenerate and $\delta \neq 0$.

4.4. Modified trace on $\operatorname{Proj}\left(\mathscr{C}^{H}\right)$. Here we discuss modified traces, or m-trace for short, defined in $[15,11]$. If $\mathscr{C}$ is a linear pivotal category, let $\operatorname{Proj}(\mathscr{C})$ be the ideal of projective objects of $\mathscr{C}$. The right partial trace of an endomorphism $f \in \operatorname{End}_{\mathscr{C}}\left(V \otimes V^{\prime}\right)$ is the endomorphism $\operatorname{ptr}(f) \in \operatorname{End}_{\mathscr{C}}(V)$ given by

$$
\operatorname{ptr}(f):=\left(\operatorname{Id}_{V} \otimes \overrightarrow{\mathrm{ev}}_{V^{\prime}}\right) \circ\left(f \otimes \operatorname{Id}_{V^{\prime *}}\right) \circ\left(\operatorname{Id}_{V} \otimes \overleftarrow{\operatorname{coev}}_{V^{\prime}}\right)
$$

Following $[15,11]$, a right $m$-trace $\mathrm{t}$ on $\operatorname{Proj}(\mathscr{C})$ is a family of linear $\operatorname{maps}\left\{\mathrm{t}_{V}: \operatorname{End}_{\mathscr{C}}(V) \rightarrow \mathbb{k} \mid V \in \operatorname{Proj}(\mathscr{C})\right\}$ satisfying:

(1) Cyclicity: $\mathrm{t}_{V}\left(f^{\prime} \circ f\right)=\mathrm{t}_{V^{\prime}}\left(f \circ f^{\prime}\right)$ for all objects $V, V^{\prime} \in \operatorname{Proj}(\mathscr{C})$ and for all morphisms $f \in \operatorname{Hom}_{\mathscr{C}}\left(V, V^{\prime}\right)$ and $f^{\prime} \in \operatorname{Hom}_{\mathscr{C}}\left(V^{\prime}, V\right)$;

(2) Partial trace: $\mathrm{t}_{V \otimes V^{\prime}}(f)=\mathrm{t}_{V}(\operatorname{ptr}(f))$ for all objects $V \in \operatorname{Proj}(\mathscr{C})$ and $V^{\prime} \in \mathscr{C}$ and for every morphism $f \in \operatorname{End}_{\mathscr{C}}\left(V \otimes V^{\prime}\right)$. 
Similarly, using the left partial trace one can define a left m-trace.

Now let $\mathscr{C}$ be the $\mathbb{C}$-linear category $\bigoplus_{\bar{\alpha} \in G} \mathscr{C}_{\bar{\alpha}}$ in which $\mathscr{C}_{\bar{\alpha}}$ is the category $\mathcal{U}_{\bar{\alpha}}$-mod of finite dimensional left $\mathcal{U}_{\bar{\alpha}}$-modules for $\bar{\alpha} \in G$. Since $\mathcal{U}$ is unimodular then there exist a right $\mathrm{m}$-trace $\mathrm{t}$ on $\operatorname{Proj}(\mathscr{C})$. Moreover, since $\mathcal{U}_{\bullet}$ is unibalanced (i.e. Proposition 4.11), then [18, Theorem 1.1] implies the right $\mathrm{m}$-trace $\mathrm{t}$ is also a left $\mathrm{m}$-trace and it is determined by

$$
\mathrm{t}_{\mathcal{U}_{\bar{\alpha}}}\left(R_{x}\right)=\mu_{\bar{\alpha}}(x)
$$

where $R_{x}$ is the right multiplication by $x \in \mathcal{U}_{\bar{\alpha}}$ seen as a morphism of left $\mathcal{U}_{\bar{\alpha}}$-module.

Recall that $\mathscr{C}^{H}=\bigoplus_{\bar{\alpha} \in G} \mathscr{C}_{\bar{\alpha}}^{H}$ is the category of weight modules over $\mathcal{U}^{H}$, see Section 2. Since $\mathcal{U} \subset \mathcal{U}^{H}$, we can consider the forgetful functor obtain by restriction $\operatorname{ResF}: \mathscr{C}_{\bar{\alpha}}^{H} \rightarrow \mathscr{C}_{\bar{\alpha}}, V^{H} \mapsto V=\operatorname{ResF}\left(V^{H}\right)$ and $f \in \operatorname{Hom}_{\mathscr{C}^{H}}\left(V^{H}, W^{H}\right) \mapsto \operatorname{ResF}(f) \in \operatorname{Hom}_{\mathscr{C}}(V, W)$. Here $V^{H}$ and $V$ have the same underlying vector space, and $V$ is obtained from $V^{H}$ by forgetting the action of $\mathfrak{H}$.

Call Proj $\left(\mathscr{C}^{H}\right)$ the ideal of projective modules of $\mathscr{C}^{H}$. We now need to assume

Axiom 5. There exists a projective module $P_{0}$ of $\mathscr{C}^{H}$ with $\operatorname{ResF}\left(P_{0}\right)$ projective in $\mathscr{C}$.

Axiom 5 implies that

Proposition 4.19. For any projective module $P$ of $\mathscr{C}^{H}$, $\operatorname{ResF}(P)$ is projective module module of $\mathscr{C}$.

Proof. Since $P$ is projective it belongs to the ideal generated by $P_{0}$. But then $\operatorname{ResF}(P)$ belongs to the ideal generated by $\operatorname{ResF}\left(P_{0}\right)$ which is the subcategory of projective objects of $\mathscr{C}$.

For $V^{H} \in \operatorname{Proj}\left(\mathscr{C}^{H}\right)$, we define a linear map

$$
\mathrm{t}_{V^{H}}: \operatorname{End}_{\mathscr{C}^{H}}\left(V^{H}\right) \rightarrow \mathbb{C}, f \mapsto \mathrm{t}_{V^{H}}(f):=\mathrm{t}_{\operatorname{ResF}\left(V^{H}\right)}(\operatorname{ResF}(f)) .
$$

Since the forgetful functor is pivotal and it commutes with the right and left partial traces and we have

Proposition 4.20. The family $\left\{\mathrm{t}_{V^{H}}\right\}_{V^{H} \in \operatorname{Proj}\left(\mathscr{C}^{H}\right)}$ determines a right and left $m$-trace on $\operatorname{Proj}\left(\mathscr{C}^{H}\right)$.

We finish by a proposition used to complete Example 2.8 and below:

Proposition 4.21. Suppose there exists a dense open subset $O$ of $G$ such that the categories $\mathscr{C}_{\bar{\alpha}}$ and $\mathscr{C}_{\bar{\alpha}}^{H}$ are semi-simple for any $\bar{\alpha} \in O$ (i.e. $\mathscr{C}$ and $\mathscr{C}^{H}$ are generically semi-simple). Also, suppose that for $\bar{\alpha} \in O$, every simple module in $\mathscr{C}_{\bar{\alpha}}$ is the image of a simple module in $\mathscr{C}_{\bar{\alpha}}^{H}$. Then

(1) If $x \in \mathcal{U}^{H}$ satifies $\rho_{V}(x)=0$ for all $V \in \mathscr{C}^{H}$ then $x=0$. 
(2) Axiom 5 holds.

Proof. Consider a non zero $x(h)=\sum x_{i} f_{i}(h) \in \widehat{\mathcal{U}^{H}}$ seen as a $W$ valued entire function. Since $x$ is not zero, there exists $\alpha \in \mathfrak{H}^{*}$ such that $x(\alpha) \neq 0$ and $\mathscr{C}_{\alpha}$ is semi-simple. Then $\mathcal{F}_{\alpha}(x) \neq 0 \in \mathcal{U}_{\bar{\alpha}} \simeq$ $\bigoplus_{i} \operatorname{End}_{\mathbb{C}}\left(V_{i}\right)$. Lifting one of the $V_{i}$ to $\mathscr{C}^{H}$, one can found a simple module $V \in \mathscr{C}^{H}$ such that $\rho_{V}\left(\mathcal{F}_{\alpha}(x)\right) \neq 0$. Let $v \in V$ be a homogeneous vector of weight $\alpha^{\prime}$ such that $\mathcal{F}_{\alpha}(x) v \neq 0 . \mathcal{F}_{\alpha}(x)$ and $x$ have the same values when $h$ belongs to $\left\{\alpha+\sum_{i} k_{i} \alpha_{i}, 0 \leq k_{i} \leq \ell-1\right\}$ thus there exists $\alpha^{\prime \prime} \in \mathfrak{H}^{*}$ such that $\alpha^{\prime \prime}-\alpha^{\prime}=\ell \lambda \in \ell \Lambda$ and $\mathcal{F}_{\alpha}(x)\left(\alpha^{\prime \prime}\right)=x\left(\alpha^{\prime \prime}\right)$. Now let $\sigma=\mathbb{C}$ endowed with the action of $\mathcal{U}^{H}$ given by $\forall u \in \mathcal{U}, \rho_{\sigma}(u)=\varepsilon(u) \operatorname{Id}_{\sigma}$ and $\rho_{\sigma}\left(H_{i}\right)=\ell \lambda\left(H_{i}\right) \operatorname{Id}_{\sigma}$. Then the vector $v^{\prime}=v \otimes 1 \in V^{\prime}=V \otimes \sigma$ satifies $\rho_{V^{\prime}}(x)\left(v^{\prime}\right) \neq 0$. Indeed, $v^{\prime}$ has weight $\alpha^{\prime \prime}$ and for $y=x\left(\alpha^{\prime \prime}\right)=$ $\mathcal{F}_{\alpha}(x)\left(\alpha^{\prime \prime}\right)=\mathcal{F}_{\alpha}(x)\left(\alpha^{\prime}\right) \in W \subset \mathcal{U}$ one has

$$
x \cdot v^{\prime}=y \cdot v^{\prime}=\left(y_{(1)} \cdot v\right) \otimes\left(\varepsilon\left(y_{(2)}\right) 1\right)=(y \cdot v) \otimes 1=\left(\mathcal{F}_{\alpha}(x) \cdot v\right) \otimes 1 \neq 0 .
$$

Now consider $\bar{\alpha} \in G$ such that $\mathscr{C}_{\bar{\alpha}}$ and $\mathscr{C}_{\bar{\alpha}}^{H}$ are semi-simple. Then the finite dimensional algebra $\mathcal{U}_{\bar{\alpha}}$ is isomorphic to the product of algebra End $_{\mathbb{C}}\left(V_{i}\right)$ for representatives $\left(V_{i}\right)_{i}$ of the isomorphism class of simple $\mathcal{U}_{\bar{\alpha}}$-modules. Lift one of these modules $V_{0}$ to a simple module $V_{0}^{H} \in \mathscr{C}_{\bar{\alpha}}^{H}$ then by semi-simplicity, $V_{0}^{H}$ is projective in $\mathscr{C}_{\bar{\alpha}}^{H}$ thus also in $\mathscr{C}^{H}$ and so is its image $V_{0}$ in $\mathscr{C}$.

Example 4.22. Combining Examples 2.3, 2.7, 4.17, Corollary 4.16 and Proposition 4.21 we can conclude that Axioms 1-5 are satisfied for the quantum group $\mathcal{U}$ associated to $\mathfrak{s l}_{2}$ at any root of unity $\xi=\exp \left(\frac{2 i \pi}{\ell}\right)$ with $\ell \geq 3$ and $\ell \notin 8 \mathbb{Z}$. Moreover, Examples 2.4, 2.8, 4.18, Corollary 4.16 and Proposition 4.21 imply that Axioms $1-5$ are satisfied for the quantum group $\mathcal{U}$ associated to simple finite dimensional complex Lie algebra for any root of unity $\xi=\exp \left(\frac{2 i \pi}{\ell}\right)$ with $\ell$ odd.

\section{TOPOLOGICAL INVARIANT OF BICHROME GRAPHS}

This section does not require the results of Section 4. It contains the construction of topological invariant corresponding from the algebra of Section 3. In particular, in this section we use the topological ribbon Hopf algebra $\widehat{\mathcal{U}^{H}}$ and the category $\mathscr{C}^{H}$ of weight modules over $\mathcal{U}^{H}$ to construct a topological universal invariant. The invariant is defined on bichrome graphs colored by both the algebra $\widehat{\mathcal{U}^{H}}$ and objects of $\mathscr{C}^{H}$. Every manifold we will consider in this paper will be oriented, every diffeomorphism of manifolds will be positive, and every link and tangle will be oriented and framed.

Let $L: \mathcal{U}^{H^{\widehat{\otimes}} n} \rightarrow \operatorname{End}_{\mathbb{C}}\left(\widehat{\mathcal{U}}^{{ }^{\dagger}}{ }^{\widehat{\otimes} n}\right)$ be the left multiplication: $L_{x}(y)=x y$.

5.1. Bichrome graphs. For a non-negative integer $n$, we describe the category $[n] \mathscr{C}^{H}$ as follows. The objects of $[n] \mathscr{C}^{H}$ are vector spaces of 
the form

$$
[n] V:=\left(\mathcal{U}^{H}\right)^{\widehat{\otimes} n} \otimes V
$$

for some object $V$ of $\mathscr{C}^{H}$. A morphism in $[n] \mathscr{C}^{H}$ from $[n] V$ to $[n] V^{\prime}$ is a morphism of topological $\widehat{\mathcal{U}^{H}}$-modules $f:[n] V \rightarrow[n] V^{\prime}$ which is of the form

$$
f=\left(L_{\xi^{q+l}} \otimes \mathrm{Id}\right) \sum_{i=1}^{m} L_{u_{i}} \otimes f_{i}
$$

for some $u_{i} \in \mathcal{U}^{\otimes n}$, a quadratic element $q$, a linear element $l$ and for some linear maps $f_{i} \in \operatorname{Hom}_{\mathbb{C}}\left(V, V^{\prime}\right), 1 \leq i \leq m$.

Let $f:[n] V \rightarrow[n] V^{\prime}$ be a non-zero morphism as in Equation (34). Then $f$ determines $q$ and $\bar{l} \in\left(G^{\prime}\right)^{n}$ the class of $l$ modulo $\left(\Lambda^{*}\right)^{n}$ uniquely. The pair $(q, \bar{l})$ is called the exponent of $f$.

As we will explain, bichrome ribbon graph mimic Turaev's definition of ribbon graphs, given in [34], but have additional information including red and blue edges and special coupons. For more on ribbon graphs, ribbon categories and their associated Reshetikhin-Turaev functors see [34]. Here

An $n$-string link is an $(n, n)$-tangle whose $i$-th incoming boundary vertex is connected to the $i$-th outgoing boundary vertex by an edge directed from bottom to top for every $1 \leq i \leq n$.

A bichrome graph is a ribbon graph with edges divided into two groups, red and blue, satisfying the following condition: for every coupon there exists a number $k \geq 0$ such that the first $k$ input legs and the first $k$ output legs are red with positive orientation, meaning incoming and outgoing respectively, while all the other ones are blue. The smoothing of a coupon with $k$ red edges is the union of $k$ parallel red strings union the coupon with its red edges removed, see Figure 1. Note this is a different smoothing than given in [10] where the blue part was removed in this process.

A $n$-string link graph is a bichrome ribbon graph $\Gamma$ in $\mathbb{R}^{2} \times[0,1]$ satisfying the following conditions: (1) the first $n$ incoming boundary vertices and the first $n$ outgoing boundary vertices are red, while all the other ones are blue and, (2) the red sub-tangle of the graph obtained by smoothing all coupons of $\Gamma$ is an $n$-string link. We say such a graph is $\left.\widehat{\mathcal{U}^{H}}, \mathscr{C}^{H}\right)$-colored if: (1) the red edges are colored by $\widehat{\mathcal{U}^{H}},(2)$ the blue edges colored by objects of $\mathscr{C}^{H}$ and (3) coupons are colored by morphisms in $[k] \mathscr{C}^{H}$ (here $k$ can vary for the different coupons).

To simplify notation, when it is clear we will say $n$-string link graph for a $\left(\widehat{\mathcal{U}^{H}}, \mathscr{C}^{H}\right)$-colored $n$-string link graph. We also consider the special kind of coloring on a $n$-string link graph: We say a $n$-string link graph

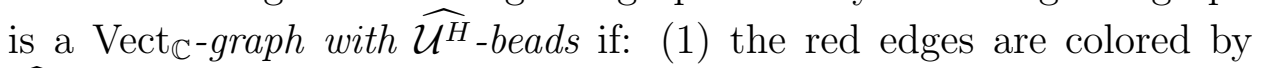
$\widehat{\mathcal{U}^{H}},(2)$ the blue edges colored by objects of $\mathscr{C}^{H},(3)$ coupons do not have any red edges and are colored by morphisms in Vect $_{\mathbb{C}}$ and (4) 

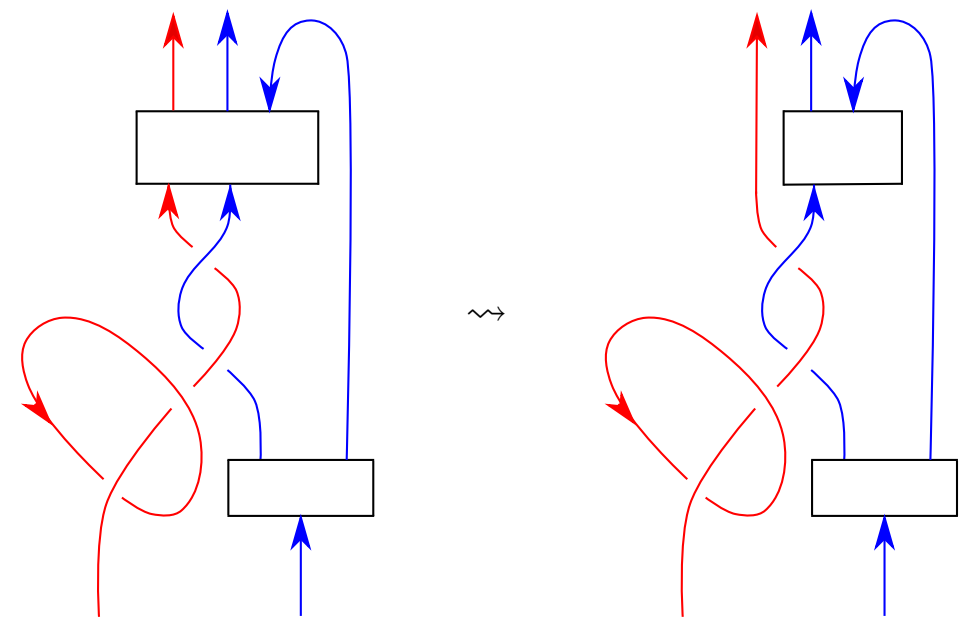

Figure 1. Smoothing of a coupon

the graph is equipped with beads which form a finite set $b$ of points on edges colored by an element of $\left(\widehat{\mathcal{U}^{H}}\right)^{\widehat{\otimes} b}$. Here and in what follows, if $S$ is a finite set with $n$ element the tensor product $V^{\otimes S}$ means $V^{\otimes n}$ where the factors are indexed by elements of $S$.

5.2. The universal invariant. Let $\mathcal{U}^{0}=\mathrm{HH}_{0}\left(\widehat{\mathcal{U}^{H}}\right):=\widehat{\mathcal{U}^{H}} /\left[\widehat{\mathcal{U}^{H}}, \widehat{\mathcal{U}^{H}}\right]$ seen as a topological $\widehat{\mathcal{U}^{H}}$-module with trivial action, and let

$$
\operatorname{tr}_{u}: \widehat{\mathcal{U}^{H}} \rightarrow \mathcal{U}^{0}
$$

be the canonical projection.

Let $\Gamma$ be a $n$-string link graph. We will now define the universal invariant associate to $\Gamma$ which is an element

$$
J(\Gamma) \in\left(\mathcal{U}^{0}\right)^{\widehat{\otimes} C} \widehat{\otimes}\left(\mathcal{U}^{H}\right)^{\widehat{\otimes} n} \otimes \operatorname{Hom}_{\mathbb{C}}\left(V, V^{\prime}\right)
$$

where $C$ is the set of closed red components of $\Gamma$. Here $V$ (respectively $V^{\prime}$ ) is the tensor product of the colors of the blue bottom (respectively top) boundary points of $\Gamma$. We will also show that $J(\Gamma)$ is $\widehat{\mathcal{U}^{H}}$-equivariant.

Choose a diagram $D$ of $\Gamma$ which is a regular projection of $\Gamma$. We define an element $J(D)$ in the following three steps.

Step 1, Construction of $B(D)$ : Using the rules of Figure 2 we put beads at the cup, the cap and the crossing (this is same as the usual universal invariant). In this figure the symbol $S^{\downarrow}(x)$ for $x \in \widehat{\mathcal{U}^{H}}$ is 


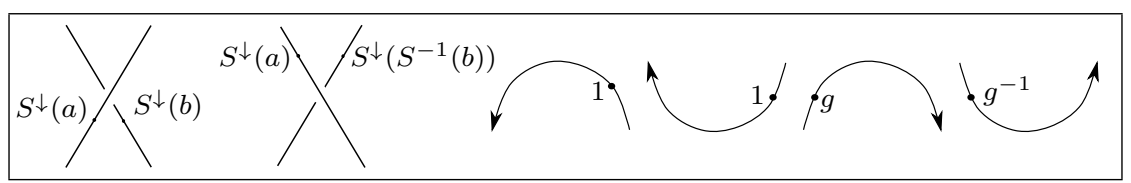

Figure 2. Place beads on the strings where $\mathcal{R}=\sum a \otimes b$

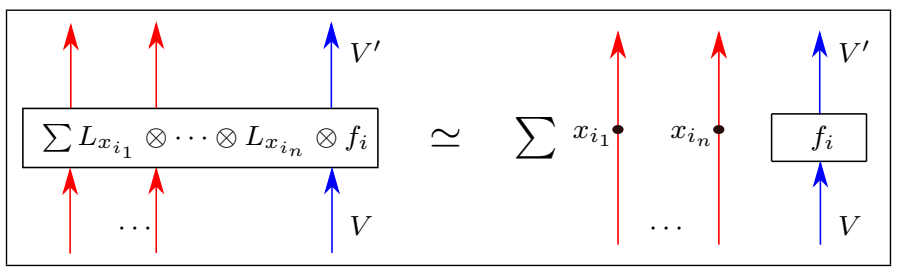

Figure 3. Smoothing a mixed coupon using beads.

defined by

$$
S^{\downarrow}(x) \downarrow=\left\{\begin{array}{r}
x \uparrow \text { if the arrow is upward, } \\
S(x) \downarrow \text { if the arrow is downward. }
\end{array}\right.
$$

Then we replace each coupon with $k$ red edges with its smoothing equipped with $k$ beads as shown in Figure 3. The result is a linear

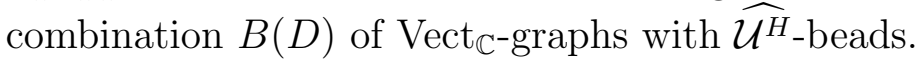

Step 2, Construction of $J_{D}^{b}$ via contraction: For each closed red component of $D$ fix a base point $b$. Using the rules of Figure 4 , we replace each bead on a blue strand colored by $V$ by using the action of $\widehat{\mathcal{U}^{H}}$ on $V$ or its transpose ${ }^{4}$. We obtain

$$
J_{D}^{b} \in\left(\mathcal{U}^{H}\right)^{\widehat{\otimes} C} \widehat{\otimes}\left(\mathcal{U}^{H}\right)^{\widehat{\otimes} n} \otimes \operatorname{Hom}_{\mathbb{C}}\left(V, V^{\prime}\right)
$$

by multiplying the beads on each red strand in order opposite to the orientation of the component (if the red component is closed start at the fixed base point) and we separately evaluate the blue part of the graph using the Penrose graphical calculus for Vect $_{\mathbb{C}^{-}}$-olored ribbon graphs.

Step 3, Definition of $J(D)$ via trace: For each closed red component apply the trace $\operatorname{tr}_{u}: \widehat{\mathcal{U}^{H}} \rightarrow \mathcal{U}^{0}$ to obtain the element

$$
J(D)=\left(\operatorname{tr}_{u}^{\otimes C} \otimes \mathrm{Id}^{\otimes n} \otimes \mathrm{Id}\right)\left(J_{D}^{b}\right) \in\left(\mathcal{U}^{0}\right)^{\widehat{\otimes} C} \widehat{\otimes}\left(\mathcal{U}^{H}\right)^{\widehat{\otimes} n} \otimes \operatorname{Hom}_{\mathbb{C}}\left(V, V^{\prime}\right) .
$$

\footnotetext{
${ }^{4}$ Remark that the convention for beads on blue shown in Figure 4 is different from [10] where we used ${ }^{t} \rho_{V}(S(x))$.
} 


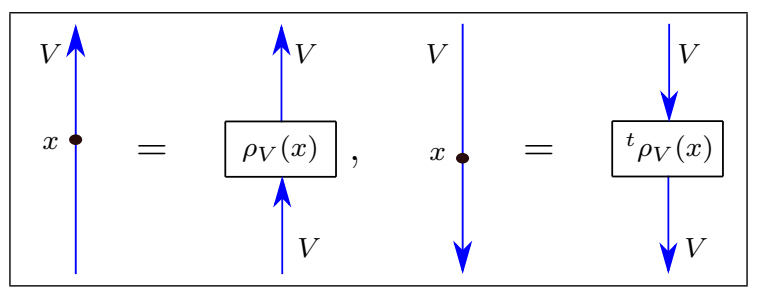

FiguRE 4. Beads on blue edges are Vect $_{\mathbb{C}}$-colored coupons.
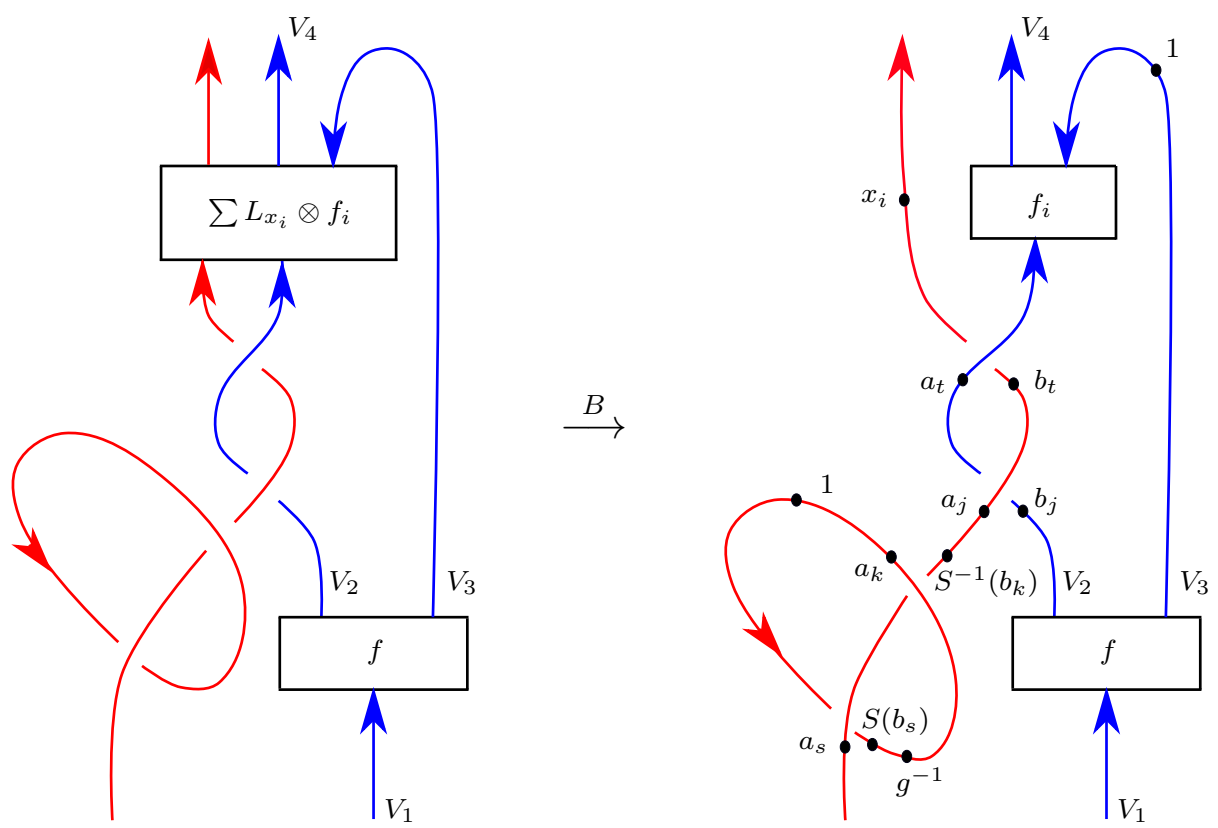

Figure 5. Putting beads on a graph $D$

The element $J(D)$ is independent of the choice of base points $b$ because the starting point of multiplying the beads on any closed red circle component does not matter as the result is sent in the quotient $\mathcal{U}^{0}$.

This construction illustrated for the diagram $D$ given in Figure 5, where $J(D)$ is an element of $\mathcal{U}^{0} \widehat{\otimes} \widehat{\mathcal{U}^{H}} \otimes \operatorname{Hom}_{\mathbb{C}}\left(V_{1}, V_{4}\right)$ given by

$$
J(D)=\sum \operatorname{tr}_{u}\left(g^{-1} S\left(b_{s}\right) 1 a_{k}\right) \otimes x_{i} b_{t} a_{j} S^{-1}\left(b_{k}\right) a_{s} \otimes F_{\mathbb{C}}(T)
$$

where $F_{\mathbb{C}}(T)=\left(\operatorname{Id}_{V_{4}} \otimes \overleftarrow{\mathrm{ev}}_{V_{3}}\right) \circ\left(f_{i} \circ \rho_{V_{2}}\left(a_{t} b_{j}\right) \otimes \operatorname{Id}_{V_{3}}\right) \circ f \in \operatorname{Hom}_{\mathbb{C}}\left(V_{1}, V_{4}\right)$ Here $F_{\mathbb{C}}$ is the Reshetikhin-Turaev functor from the category of Vect $_{\mathbb{C}^{-}}$ colored ribbons graphs to the category of finite dimensional vector spaces Vect $_{\mathbb{C}}$.

The following theorem is an adapted version of the universal invariant of $[20,27,22]$ :

Theorem 5.1. The element $J(D)$ computed above on does not depend on the choice of $D$ and is an isotopy invariant $J(\Gamma)$ of $\Gamma$ in $\mathbb{R}^{2} \times[0,1]$. 
Furthermore $J(\Gamma)$ is $\widehat{\mathcal{U}}^{H}$-equivariant, i.e.,

$J(\Gamma)\left(u_{(1)} \otimes \cdots \otimes u_{(k)} \otimes \rho_{V}\left(u_{(k+1)}\right)\right)=\left(u_{(1)} \otimes \cdots \otimes u_{(k)} \otimes \rho_{V^{\prime}}\left(u_{(k+1)}\right)\right) J(\Gamma)$

for all $u \in \widehat{\mathcal{U}^{H}}$ where $\Delta^{k+1}(u)=u_{(1)} \otimes \cdots \otimes u_{(k+1)}$ and $k$ equals $n$ plus the number of closed red components of $\Gamma$.

Proof. We will use the symbol $\equiv$ to relate two Vect $\mathbb{C}_{-}$graphs with $\widehat{\mathcal{U}}_{-}$ beads whose images are equal under $F_{\mathbb{C}}$.

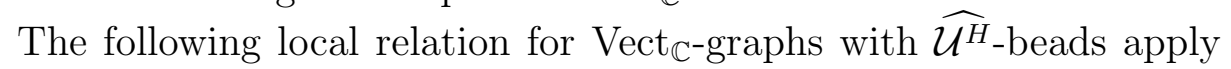
for any color of their edges:

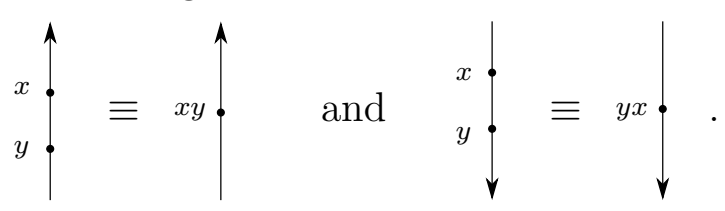

Also beads can freely move around cap and cup with any orientation:

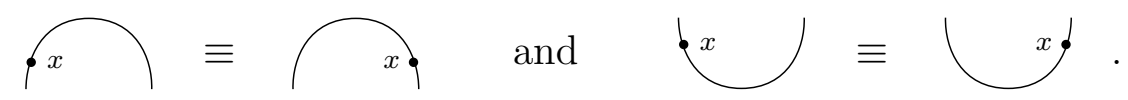

Using these relations the invariance of $J(\Gamma)$ under Redeimeister's moves follows the proof of [27] using the properties of the R-matrix. The invariance for the local move of a strand sliding over (or under) a coupon follows from the $\widehat{\mathcal{U}^{H}}$-equivariance of the morphisms coloring the coupon. We will prove the invariance of the local move given in Figure 6, the other moves are proved similarly. After putting the beads and smoothing the coupon the associated equality is

$$
\sum a_{j} a_{i} \otimes x b_{i} \otimes f \circ b_{j}=\sum a_{t} a_{s} \otimes b_{s} x \otimes b_{t} \circ f .
$$

This equality is equivalent to

$$
\left(1 \otimes \sum x \otimes f\right) \mathcal{R}_{13} \mathcal{R}_{12}=\mathcal{R}_{13} \mathcal{R}_{12}\left(1 \otimes \sum x \otimes f\right) .
$$

We consider the right hand side of Equality (38), one gets

$$
\begin{aligned}
\mathcal{R}_{13} \mathcal{R}_{12}\left(1 \otimes \sum x \otimes f\right) & =(\operatorname{Id} \otimes \Delta)(\mathcal{R})\left(1 \otimes \sum x \otimes f\right) \\
& =\left(\sum a_{i} \otimes \Delta\left(b_{i}\right)\right)\left(1 \otimes \sum x \otimes f\right) \\
& =\sum a_{i} \otimes \Delta\left(b_{i}\right)\left(\sum x \otimes f\right) \\
& =\sum a_{i} \otimes\left(\sum x \otimes f\right) \Delta\left(b_{i}\right) \\
& =\left(1 \otimes \sum x \otimes f\right)(\operatorname{Id} \otimes \Delta)(\mathcal{R}) \\
& =\left(1 \otimes \sum x \otimes f\right) \mathcal{R}_{13} \mathcal{R}_{12} .
\end{aligned}
$$

In the third equality we used $\sum L_{x} \otimes f$ being $\widehat{\mathcal{U}}^{H}$-invariant. Hence Equality (38) holds. 

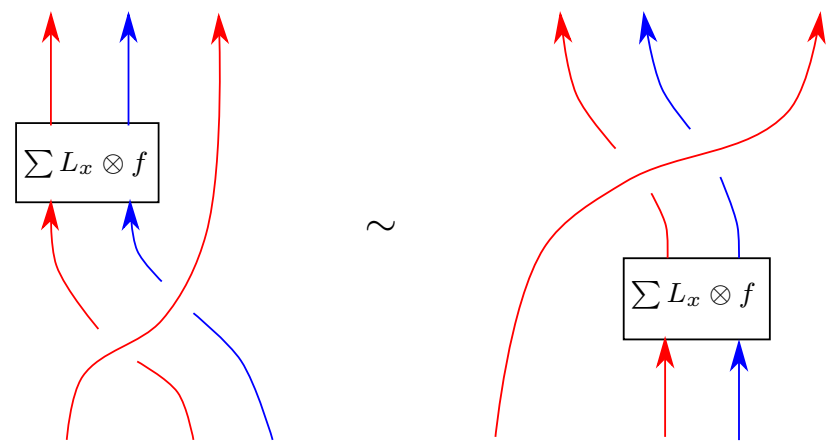

FiguRE 6. Moving at local coupon

As claimed above, the element $J(D)$ is independant of the choice of base point: indeed, if one move the base point so that it pass a bead colored by $a \in \mathcal{U}^{H}$ the two corresponding values for $J(D)$ will be the same except for a factor which will be $\operatorname{tr}_{u}(x a)$ in one case and $\operatorname{tr}_{u}(a x)$ on the other case but these two elements are equal in $\mathcal{U}^{0}$ so moving the base does not change $J(D)$.

We now prove that $J(\Gamma)$ is $\widehat{\mathcal{U}^{H}}$-equivariant, i.e., Equation (35) holds. One can compute the left hand side of Equation (35) by applying $F_{\mathbb{C}}$ to the diagram $B(D)$ where some beads colored by the $u_{(i)}$ have been threaded at the intersection point of $D$ with an horizontal line near the bottom of $D$. Similarly, the right hand side is the image by $F_{\mathbb{C}}$ of the diagram $B(D)$ with some $u_{(i)}$ beads along a horizontal line near the top of $D$. For a horizontal line at any level (not intersecting coupons) we can define similarly a diagrams obtain from $B(D)$ with some $S^{\downarrow}\left(u_{(i)}\right)$ beads at the intersection point of $\Gamma$ with the horizontal line.

We claim that all diagrams obtained by this process will have the same image by $F_{\mathbb{C}}$. Indeed, it is enough to see this is true when one moves the horizontal line to pass an elementary diagram. Except for red caps and cups, this follows from the $\widehat{\mathcal{U}}^{H}$-equivariance of the braiding given by $\tau \mathcal{R}$, of the morphisms coloring coupons and of the (co)evalutations in $\mathscr{C}^{H}$. For red cups and caps it is a direct computation ; we develop here the case of red caps (see Figure 7): Assume the horizontal line under the cap intersect $\Gamma$ at $N+2$ points and at $N$ points when the line is above the cap. If the cap is joining the $i^{\text {th }}$ and the $i+1^{\text {th }}$ strands, we can write $\Delta^{N} u=u_{(1)} \otimes \cdots \otimes u_{(i-1)} \otimes a \otimes u_{(i+1)} \otimes \cdots \otimes u_{(N+1)}$. Then $\Delta^{N-1} u=u_{(1)} \otimes \cdots \otimes u_{(i-1)} \otimes \varepsilon(a) \otimes u_{(i+1)} \otimes \cdots \otimes u_{(N+1)}$ and $\Delta^{N+1} u=u_{(1)} \otimes \cdots \otimes u_{(i-1)} \otimes a_{(1)} \otimes a_{(2)} \otimes u_{(i+1)} \otimes \cdots \otimes u_{(N+1)}$. Then the statements for the red caps (see Figure 7 ) hold from the equalities $S\left(a_{(1)}\right) a_{(2)}=\varepsilon(a)$ and $S\left(a_{(2)}\right) g a_{(1)}=g S^{-1}\left(a_{(2)}\right) a_{(1)}=\varepsilon(a) g$. The similar statements for the red cups hold from the first equality above and $a_{(2)} g^{-1} S\left(a_{(1)}\right)=\varepsilon(a) g^{-1}$ for $a \in \widehat{\mathcal{U}^{H}}$. 


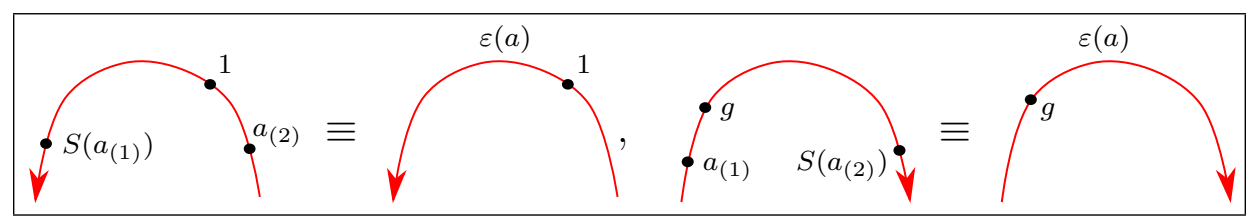

FigURE 7. Equivariance for red caps

Lemma 5.2. Let $\widehat{\mathcal{U}}_{0}$ be the weight 0 subspace of $\widehat{\mathcal{U}^{H}}$. Then

$$
\mathcal{U}^{0} \cong \widehat{\mathcal{U}}_{0} /\left(\left[\mathcal{U}, \widehat{\mathcal{U}^{H}}\right] \cap \widehat{\mathcal{U}}_{0}\right) .
$$

Proof. First $\widehat{\mathcal{U}^{H}}$ is a weight module and if $x$ is homogeneous of degree $\lambda \neq 0$, then there exists $h \in \mathfrak{H}$ such that $\lambda(h)=1$ and then $x=[h, x]$ is a commutator. Hence only weight 0 elements contribute to $\mathcal{U}^{0}$. Next, remark that if $x=f(h) w \in \widehat{\mathcal{U}^{H}}$ and $y \in \widehat{\mathcal{U}^{H}}$ have opposite weights, then $f(h) w y=w y f(h)$ and

$$
[x, y]=[f(h) w, y]=f(h) w y-y f(h) w=[w, y f(h)] \in\left[\mathcal{U}, \widehat{\mathcal{U}^{H}}\right] .
$$

5.3. Value of the invariant. Here we describe some properties of the invariant $J$ of the previous section. Let $D$ be a planar diagram representing a $n$-string graph $\Gamma$ in $\mathbb{R}^{2} \times[0,1]$ having $m$ closed red components. In the rest of the paper we will assume that all $n$-string graph will be homogenous: meaning that each blue edge is colored homogenous object of $\mathscr{C}^{H}$, see (7). In particular, we use the following notation: the $j^{\text {th }}$ blue edge is associated with an element $\bar{\beta}_{j} \in G$ such that this edge is colored by an object of $\mathscr{C}_{\bar{\beta}_{j}}^{H}$.

Choose a base point $b$ for each closed red component of the diagram

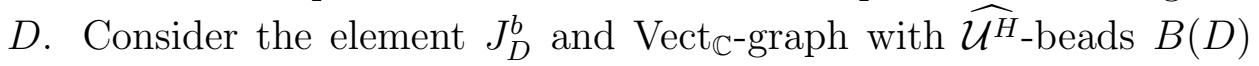
given in Section 5.2. The diagram $B(D)$ consists of $m+n$ red components and a blue graph $D_{b l}$ with beads and $n_{D_{b l}}$ edges. We numbered the components and edges of $D$ starting with the $m$ red closed components then the $n$ ordered open red components and finally the blue edges. As in Section 5.2 we express

$$
J(\Gamma)=\left(\operatorname{tr}_{u}^{\otimes m} \otimes \operatorname{Id}^{\otimes n} \otimes \mathrm{Id}\right)\left(J_{D}^{b}\right) \in \mathcal{U}^{0 \widehat{\otimes} m} \widehat{\otimes}\left(\mathcal{U}^{H}\right)^{\widehat{\otimes} n} \otimes \operatorname{Hom}_{\mathbb{C}}\left(V, V^{\prime}\right)
$$

where $J_{D}^{b}=\sum w_{b_{1}} \otimes \cdots \otimes w_{b_{m}} \otimes y_{1} \otimes \cdots \otimes y_{n} \otimes F_{\mathbb{C}}\left(D_{b l}\right)$ depends on the choice $b$.

The element $J_{D}^{b}$ can be seen as a vector-valued entire function with $r(m+n)$ variables: indeed, we can associate to the $i^{\text {th }}$ red component, the set of variables

$$
h_{i}=\left(H_{1, i}, \ldots, H_{r, i}\right) .
$$

Recall that a morphism $f:[k] V \rightarrow[k] V^{\prime}$ determines a pair $(q, \bar{l})$ called the exponent determined by Equation (34). For each coupon $c$ of $D$ 
with $k$ red legs let $(q, l)$ be the exponent corresponding to the morphism in $c$ with $\left(\xi^{q}, l\right) \in \mathcal{Q}_{k} \times\left(G^{\prime}\right)^{k}$. For such a coupon, let $i_{1}, i_{2}, \ldots, i_{k} \in$ $\{1, \ldots, m+n\}$ be the labels (with possible repetition) of its adjacent red edges and let $q(c, D)=q\left(h_{i_{1}}, \ldots, h_{i_{k}}\right)$ then $\xi^{q(c, D)} \in \mathcal{Q}_{m+n}$ and $l(c, D)=l\left(h_{i_{1}}, \ldots, h_{i_{k}}\right) \in\left(G^{\prime}\right)^{m+n}$. Finally, let

$$
\mathrm{Q}_{D}^{c}=\sum_{c \text { coupon }} q(c, D) \text { and } \bar{L}_{D}^{c}=\sum_{c \text { coupon }} l(c, D) .
$$

Next, we will use the elements $\mathrm{Q}_{D}^{c}$ and $\bar{L}_{D}^{c}$ to assign an exponent to $J_{D}^{b}$. This exponent is a sum of three contributions: the first is a quadratic element coming from the crossing between red strands, the second is a linear element coming from bichrome crossings and the last is coming from the coupons.

Let $\mathrm{lk}=\left(\mathrm{lk}_{i j}\right)_{1 \leq i, j \leq m+n+n_{D_{b l}}}$ be the linking matrix associated $D$. Remark that the first $(m+n) \times(m+n)$ block of lk does not depend on the planar projection but the second does: the coefficient $(i, j)$ is the sum of the sign of crossings of the planar diagram where the strand $j$ is above the strand $i$ (see [6]). Recall the element $\mathbf{Q}=\mathrm{Q}\left(h_{1}, h_{2}\right)=$ $\sum_{i} c_{i} \otimes c_{i}^{\prime} \in \Lambda^{*} \otimes_{\mathbb{Z}} \Lambda^{*} \subset \mathfrak{H} \otimes \mathfrak{H}$ which corresponds to the symmetric bilinear form $\mathrm{B}$ on $\Lambda$ and $\mathcal{H}=\xi^{\mathrm{Q}\left(h_{1}, h_{2}\right)}$. Let

$$
\begin{aligned}
& \mathrm{Q}_{D}=\sum_{1 \leq k, s \leq m+n} \mathrm{lk}_{k s} \mathrm{Q}\left(h_{k}, h_{s}\right), \quad \text { so } \xi^{\mathrm{Q}_{D}} \in \mathcal{Q}_{m+n}, \\
& \bar{L}_{D}=\sum_{1 \leq k \leq m+n<s \leq m+n+n_{D_{b l}}}\left(\mathrm{lk}_{k s}+\mathrm{lk}_{s k}\right) \mathrm{Q}\left(\bar{\beta}_{s}, h_{k}\right) \in\left(G^{\prime}\right)^{m+n} .
\end{aligned}
$$

The following theorem is analogous to [17, Theorem 3.4]:

Theorem 5.3. Let $L_{D}$ and $L_{D}^{c}$ be any representatives in $\mathfrak{H}^{(m+n)}$ of $\bar{L}_{D}$ and $\bar{L}_{D}^{c}$ in $\left(G^{\prime}\right)^{m+n}=\left(\mathfrak{H} / \Lambda^{*}\right)^{m+n}$, respectively and let $P_{D}=\mathrm{Q}_{D}+$ $\mathrm{Q}_{D}^{c}+L_{D}+L_{D}^{c}$. Then the element $\xi^{P_{D}}$ is independent of the choice of these representatives up to a unit in $\mathcal{U}^{\otimes m+n}$ (i.e. $\xi$ to an integral linear combination of the $H_{i, j}$ ) and

$$
J_{D}^{b} \in \xi^{P_{D}} \mathcal{U}^{\otimes m+n} \otimes \operatorname{Hom}_{\mathbb{C}}\left(V, V^{\prime}\right) .
$$

Proof. The first statement of the theorem follows from Remark 3.8. To prove Equation (42) notice that the elements $H_{i, j}$ form a commutative algebra and by Lemma 3.9 power quadratic and power linear elements commutes with $\mathcal{U}$ so all contributions to the exponent can be added in any order. We just have to check that the contribution of crossings is given by the elements $\mathrm{Q}_{D}$ and $\bar{L}_{D}$.

At each crossing point of type $(k, s)$ in the diagram of $B(D)$ with beads for $1 \leq k, s \leq m+n$, i.e., a red-red type crossing point, the Cartan part of the $\mathcal{R}$-matrix gives us the factor

$$
\xi^{\varepsilon \mathrm{Q}\left(h_{k}, h_{s}\right)} \in \mathcal{Q}_{m+n}
$$


where $\varepsilon \in\{ \pm 1\}$ is the sign of the crossing. Summing all these contributions, we get a factor

$$
\xi^{\mathrm{lk}_{k s} \mathrm{Q}\left(h_{k}, h_{s}\right)} \in \mathcal{Q}_{m+n} .
$$

Next we consider a crossing point $(k, s)$ in the the diagram of $B(D)$ for $1 \leq k \leq m+n, m+n<s \leq m+n+n_{D_{b l}}$, i.e., a red-blue type crossing point. Suppose that the color of the blue strand is $V \in \mathscr{C}_{\bar{\beta}}^{H}$ for $\bar{\beta} \in G$. Then any weight $\lambda$ of $V$ is congruent to $\bar{\beta}$ modulo $\Lambda$ and the Cartan part of the element $\mathcal{R}$-matrix acting on the $\lambda$ weight space of $V$ gives us a factor (see Remark 3.8)

$$
\xi^{\varepsilon_{k s} \mathrm{Q}\left(\lambda, h_{k}\right)} \in \xi^{\varepsilon_{k s} \mathrm{Q}\left(\bar{\beta}, h_{k}\right)} \mathcal{U}^{\otimes m+n} \subset \mathcal{L}_{m+n} \mathcal{U}^{\otimes m+n} .
$$

So the $k^{\text {th }}$ red component and the $s^{\text {th }}$ blue component give the factor

$$
\xi^{\left(\mathrm{lk}_{k s}+\mathrm{lk}_{s k}\right) \mathrm{Q}\left(\bar{\beta}, h_{k}\right)} \in \mathcal{L}_{m+n} \mathcal{U}^{\otimes m+n} .
$$

Lemma 5.4. Let $D$ be as above and let $\overleftarrow{D}$ be the same diagram with the orientation of the $i^{\text {th }}$ closed red component reversed $(1 \leq i \leq m)$. Here we assume that this component does not pass through a coupon. Then $J_{\stackrel{\leftarrow}{b}}^{b}$ is obtained from $J_{D}^{b}$ by applying the antipode or its inverse to the $i^{\text {th }}$ factor, depending if the strand is oriented to the top or to the bottom at the base point.

Proof. We can split the $i^{\text {th }}$ component into a sequence of segments whose end points are the extremal points (critical points of the second coordinate) $p_{1}, \ldots, p_{2 k}$ at cups and caps (cups and caps alternate when one follows the component).

Let us assume first that the base point is on the segment $\left[p_{2 k}, p_{0}\right]$ which is oriented upward. Then multiplying together the beads on each segment, the contribution to the $i^{\text {th }}$ factor of $J_{D}^{b}$ has the form

$$
\begin{aligned}
X & =S^{\downarrow}\left(a_{2 k}\right) g^{-\varepsilon_{2 k}} S^{\downarrow}\left(a_{2 k-1}\right) g^{\varepsilon_{2 k-1}} S^{\downarrow}\left(a_{2 k-2}\right) \cdots g^{-\varepsilon_{2}} S^{\downarrow}\left(a_{1}\right) g^{\varepsilon_{1}} S^{\downarrow}\left(a_{0}\right) \\
& =a_{2 k} g^{-\varepsilon_{2 k}} S\left(a_{2 k-1}\right) g^{\varepsilon_{2 k-1}} a_{2 k-2} \cdots g^{-\varepsilon_{2}} S\left(a_{1}\right) g^{\varepsilon_{1}} a_{0}
\end{aligned}
$$

where (see also Figure (2))

(1) $S^{\downarrow}\left(a_{j}\right)$ is the product of the beads on the $j^{\text {th }}$ segment $\left[p_{j}, p_{j+1}\right]$ where the $a_{j}$ are products of factors of $\mathcal{R}$ or $\mathcal{R}^{-1}$,

(2) the $j^{\text {th }}$ segment $\left[p_{j}, p_{j+1}\right]$ is oriented upward if $j$ is even and downward if $j$ is odd,

(3) $\varepsilon_{j}$ is 0 if the cap/cup is oriented to the left and 1 if it is oriented to the right.

Then the corresponding contribution to the $i^{\text {th }}$ factor of $J_{\stackrel{\leftarrow}{b}}^{b}$ has the form

$$
X^{\prime}=S\left(a_{0}\right) g^{\varepsilon_{1}^{\prime}} a_{1} g^{-\varepsilon_{2}^{\prime}} \cdots S\left(a_{2 k-2}\right) g^{\varepsilon_{2 k-1}^{\prime}} a_{2 k-1} g^{-\varepsilon_{2 k}^{\prime}} S\left(a_{2 k}\right)
$$


where $\varepsilon_{j}^{\prime}=1-\varepsilon_{j}$ because a left oriented cap/cup become a right oriented cap/cup (once again see Figure (2)). Then the lemma follows from the fact that for each $j$

$$
S\left(g^{-\varepsilon_{2 j}} S\left(a_{2 j-1}\right) g^{\varepsilon_{2 j-1}}\right)=g^{-\varepsilon_{2 j-1}} S^{2}\left(a_{2 j-1}\right) g^{\varepsilon_{2 j}}=g^{\varepsilon_{2 j-1}^{\prime}} a_{2 j-1} g^{-\varepsilon_{2 j}^{\prime}},
$$

so we have $X^{\prime}=S(X)$.

Since changing the orientation twice recover the initial diagram, and $X=S^{-1}\left(X^{\prime}\right)$, we get that in case the base point is on a downward segment, the new invariant is obtained by applying $S^{-1}$.

The following proposition established the universal character of the red colored strands:

Proposition 5.5. Let $\Gamma$ be a bichrome graph and $\Gamma_{V}$ be obtained by replacing the $i^{\text {th }}$ colored red strand by a blue strand colored by $V \in \mathscr{C}^{H}$ then

(1) if the strand is closed, $J\left(\Gamma_{V}\right)$ is obtained from $J(\Gamma)$ by applying $\operatorname{tr}_{\mathbb{C}}^{V} \circ \rho_{V}$ on the factor of $\mathcal{U}^{0}$ coresponding to this strand.

(2) if the strand is the last open red strand, then $J\left(\Gamma_{V}\right)$ is obtained from $J_{\Gamma}$ by applying $\rho_{V}$ on the last factor of $\mathcal{U}^{H}$.

Proof. This is a consequence from the fact that beads can be collected without paying attention to the color of the strand by using the relations (36) and (37). Then Penrose graphical calculus in Vect $\mathbb{C}_{\mathbb{C}}$ does not depends of the embedding of a strand because the braiding in vect $\mathbb{C}_{\mathbb{C}}$ is trivial so we can assume that the strand is either a simple circle or a vertical string.

\section{INVARIANT OF 3-MANIFOLDS}

The goal of this section is to define an invariant of the compatible triples $(M, \Gamma, \omega)$ where, loosely speaking, $M$ is a 3 -manifold, $\Gamma$ is a closed bichrome graph inside $M$ and $\omega \in H^{1}(M \backslash \Gamma, G)$. The main ingredients of the construction are the universal invariant defined in previous section, the discrete Fourier transforms, the graded integral, the modified trace and the modified integral.

6.1. Compatible triple. A closed bichrome ribbon graph is a bichrome ribbon graph with no boundary vertices. Let $\Gamma$ be a $n$-string link graph in $M=\mathbb{R}^{2} \times[0,1]$ or a closed bichrome graph embedded in any oriented closed 3-manifold $M$. Let $\widetilde{\Gamma}$ be the smoothing of $\Gamma$ in $M$. Let $\omega \in H^{1}(M \backslash \widetilde{\Gamma}, G)$. We now defined the notion of a compatible triple, first in a simple situation and then the general case. First, we consider the situation with the following two requirements: 1) the bilinear form $B$ (involve in the braiding) is non degenerate and 2) every closed red component of $\Gamma$ is a framed knot with no coupons. With these requirements we say the triple $(M, \Gamma, \omega)$ is compatible if: 
(1) For each blue edge $e$ of $\Gamma$ colored by $V \in \mathscr{C}_{g}^{H}$ with oriented meridian $m_{e}$, one has $g=\omega\left(m_{e}\right)$.

(2) For each $i^{\text {th }}$ closed red component of $\Gamma$ with oriented parallel $\ell_{i}$, one has $\omega\left(\ell_{i}\right)=0 \in G$.

For the general case, consider the element $\vec{\omega}_{m} \in G^{m+n}$ obtained by evaluation of $\omega$ on the meridians of the $m+n$ red components of $\widetilde{\Gamma}$. By Lemma 3.7, the quadratic element $\mathrm{Q}_{\Gamma}^{c}$ of Equation (39) produces an element $\widetilde{Q}_{\Gamma}^{c}\left(\vec{\omega}_{m}, h_{\bullet}\right) \in\left(G^{\prime}\right)^{m+n}$. Let $f_{i}^{c} \in G^{\prime}$ be the $i^{\text {th }}$ component of $\widetilde{\mathrm{Q}}_{\Gamma}^{c}\left(\vec{\omega}_{m}, h_{\bullet}\right)+\bar{L}_{\Gamma}^{c}$. Similarly, for $\mathrm{Q}\left(h_{1}, h_{2}\right)$ the exponant of $\mathcal{H}$ and for any $\bar{\alpha} \in G$, we consider the element $\mathrm{Q}(\bar{\alpha}, h) \in G^{\prime}$. Explicitly, if $\mathrm{Q}=\sum_{i} c_{i}(h) \otimes c_{i}^{\prime}(h)$, then $\mathrm{Q}(\bar{\alpha}, h)=\sum_{i} c_{i}(\bar{\alpha}) c_{i}^{\prime}$ where the $c_{i}(\bar{\alpha}) \in \mathbb{C} / \mathbb{Z}$ are complex numbers modulo integers. Then we say the triple $(M, \Gamma, \omega)$ is compatible if:

(1) For each blue edge $e$ of $\Gamma$ colored by $V \in \mathscr{C}_{\bar{\beta}}^{H}$ with oriented meridian $m_{e}$, one has $\bar{\beta}=\omega\left(m_{e}\right)$.

(2) For each $i^{\text {th }}$ red closed component of $\Gamma$ with oriented parallel $\ell_{i}$, one has $2 \mathrm{Q}\left(\omega\left(\ell_{i}\right), h\right)+f_{i}^{c}=0 \in G^{\prime}$.

If $(M, \Gamma, \omega)$ is a compatible triple where $M$ is a closed 3-manifold presented by surgery on a link $L \subset S^{3}$ then $S^{3} \backslash(\widetilde{\Gamma} \cup L) \subset M \backslash \widetilde{\Gamma}$ and in what follows we will still denote $\omega$ as the restriction of the cohomology class $\omega$ in $H^{1}\left(S^{3} \backslash(\widetilde{\Gamma} \cup L), G\right)$.

Proposition 6.1. Let $(M, \Gamma, \omega)$ be a compatible triple where $M$ is a be an oriented closed 3-manifold presented by surgery on a link $L \subset S^{3}$. Then $\left(S^{3}, \Gamma \cup L, \omega\right)$ is also a compatible triple where the components of $L$ are all red.

Proof. Condition (1) of a compatible triple is still satisfied because the blue edges $\Gamma$ and $\Gamma \cup L$ are the same. We need to check condition (2) for the red components of $L$. The surgery link $L$ has no coupon and its parallels bound discs in $M \backslash \widetilde{\Gamma}$. Hence for each parallel $\ell_{i}$ of a component of $L, \omega\left(\ell_{i}\right)=0$. Hence the triple $\left(S^{3}, \Gamma \cup L, \omega\right)$ is compatible.

Kirby's Theorem (given in [23]) loosely says that two surgery presentations of a manifold can be connected by a sequence of isotopies and Kirby moves. Consider the Kirby move given in Figure 8. Notice that the Kirby 0 and II moves only occur on closed red components without coupon.

Theorem 6.2. Let $(M, \Gamma, \omega)$ and $\left.\left(M^{\prime}, \Gamma^{\prime}, \omega^{\prime}\right)\right)$ be compatible triple where $M$ and $M^{\prime}$ are oriented closed 3-manifolds. Let $M$ and $M^{\prime}$ be presented by surgery on the links $L$ and $L^{\prime}$, respectively. Suppose $f: M \rightarrow M^{\prime}$ is an be an orientation-preserving diffeomorphism such that $f(\Gamma)=\Gamma^{\prime}$ as framed graph and $f^{*}\left(\omega^{\prime}\right)=\omega$. Then there exists a finite sequence of isotopies, Kirby 0, Kirby I, Kirby II moves (see Figure 8) relating 

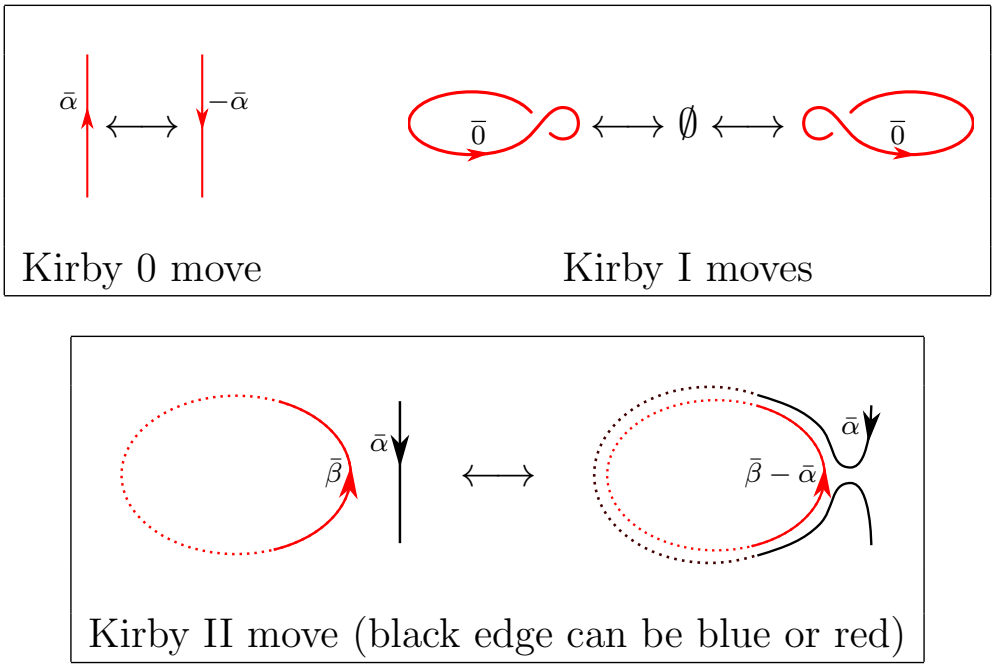

The label on edges is the value of $\omega$ on their oriented meridian.

FiguRE 8. Colored Kirby moves

$\left(S^{3}, \Gamma \cup L, \omega\right)$ and $\left(S^{3}, \Gamma^{\prime} \cup L^{\prime}, \omega^{\prime}\right)$ such that the induced isomorphism is isotopic to $f$.

Proof. In [30], the Kirby theorem is extended to the case of manifolds containing graphs. It is easy to see that the condition $f^{*}\left(\omega^{\prime}\right)=\omega$ implies that this extension give a sequence of moves relating $\left(S^{3}, \Gamma \cup\right.$ $L, \omega)$ and $\left(S^{3}, \Gamma^{\prime} \cup L^{\prime}, \omega^{\prime}\right)$. Note a similar result was considered in Theorem 5.2 of $[6]$.

6.2. Invariant of 3 -manifolds. Let $\Gamma$ be a $n$-string link graph with $m$ red closed components. Let $D$ be a planar diagram representing $\Gamma$ with a choice of base point $b$ for each closed red component. Recall we constructed in Step 2 of Subsection 5.2 the element

$$
J_{D}^{b} \in\left(\mathcal{U}^{H}\right)^{\widehat{\otimes} m+n} \otimes \operatorname{Hom}_{\mathbb{C}}\left(V, V^{\prime}\right) \simeq \mathscr{C}^{\omega}\left(h_{\bullet}\right) \otimes W^{\otimes m+n} \otimes \operatorname{Hom}_{\mathbb{C}}\left(V, V^{\prime}\right) .
$$

So $J_{D}^{b}$ is a vector-valued entire function with set of variables $h_{\bullet}=$ $\left\{H_{i, j}\right\}_{i=1, \ldots, r, j=1, \ldots, m+n}$ which are a basis of $\mathfrak{H}^{(m+n)}$.

Lemma 6.3. Suppose $\Gamma$ is equipped with a compatible cohomology class $\omega$. Let $\vec{\alpha}=\left(\bar{\alpha}_{1}, \ldots, \bar{\alpha}_{m+n}\right)$ be the values of $\omega$ on the meridians of the red components. Then $J_{D}^{b}$ is $\ell$-periodic in the first $r m$ variables $\left\{H_{i, j}\right\}_{i=1, \ldots, r, j=1, \ldots, m}$ on $\Lambda_{\vec{\alpha}}$.

Proof. Theorem 5.3 implies

$$
J_{D}^{b} \in \xi^{P_{D}} \mathcal{U}^{\otimes m+n} \otimes \operatorname{Hom}_{\mathbb{C}}\left(V, V^{\prime}\right) \subset \mathcal{Q}_{m+n} \mathcal{L}_{m+n} \mathcal{U}^{\otimes m+n} \otimes \operatorname{Hom}_{\mathbb{C}}\left(V, V^{\prime}\right)
$$

where $P_{D}=\mathrm{Q}_{D}+\mathrm{Q}_{D}^{c}+L_{D}+L_{D}^{c} \in S\left(\mathfrak{H}^{(m+n)}\right)$. Let us denote $M=$ $\mathbb{R}^{2} \times[0,1]$. Now we show that the compatibility of $\omega \in H^{1}(M \backslash \Gamma ; G)$ implies that the function $\xi^{P_{D}}$ is $\ell$-periodic in the first $r m$ variables on $\Lambda_{\vec{\alpha}}$. 
We check the periodicity of $J_{D}^{b}\left(h_{1}, \ldots, h_{m+n}\right)$ in the set of variables $h_{i}=\left(H_{1, i}, \ldots, H_{r, i}\right)$ for $1 \leq i \leq m$ by computing the evaluation of $\xi^{P_{D}}$ at $\alpha+\ell \lambda_{i}$ with $\alpha \equiv \vec{\alpha} \bmod \Lambda^{\bar{m}+n}$ and $\lambda_{i}=(0, \ldots, \lambda, \ldots, 0) \in\left(\mathfrak{H}^{*}\right)^{m+n}$ ( $\lambda$ at the $i^{\text {th }}$ position) for some $\lambda \in \Lambda$. In the following we used the polarisation of quadratic element defined in Equation (23). One has

$$
\begin{aligned}
& P_{D}\left(\alpha+\ell \lambda_{i}\right) \\
& =\mathrm{Q}_{D}\left(\alpha+\ell \lambda_{i}\right)+\mathrm{Q}_{D}^{c}\left(\alpha+\ell \lambda_{i}\right) \\
& \quad+L_{D}\left(\alpha+\ell \lambda_{i}\right)+L_{D}^{c}\left(\alpha+\ell \lambda_{i}\right) \\
& =P_{D}(\alpha)+\mathrm{Q}_{D}\left(\ell \lambda_{i}\right)+\mathrm{Q}_{D}^{c}\left(\ell \lambda_{i}\right)+\ell \widetilde{\mathrm{Q}}_{D}\left(\alpha, \lambda_{i}\right) \\
& \quad+\ell \widetilde{\mathrm{Q}}_{D}^{c}\left(\alpha, \lambda_{i}\right)+\ell L_{D}\left(\lambda_{i}\right)+\ell L_{D}^{c}\left(\lambda_{i}\right) \\
& =P_{D}(\alpha)+\mathrm{Q}_{D}\left(\ell \lambda_{i}\right)+\mathrm{Q}_{D}^{c}\left(\ell \lambda_{i}\right)+X
\end{aligned}
$$

where $X \bmod \ell \mathbb{Z}$ is given by

$$
\bar{X}=\widetilde{\mathrm{Q}}_{D}\left(\ell \vec{\alpha}, \lambda_{i}\right)+\widetilde{\mathrm{Q}}_{D}^{c}\left(\ell \vec{\alpha}, \lambda_{i}\right)+\ell \bar{L}_{D}\left(\lambda_{i}\right)+\ell \bar{L}_{D}^{c}\left(\lambda_{i}\right) .
$$

It is clear that $\mathrm{Q}_{D}\left(\ell \lambda_{i}\right)+\mathrm{Q}_{D}^{c}\left(\ell \lambda_{i}\right)=0 \bmod \ell \mathbb{Z}$. Now recall that $\mathrm{Q}=$ $\mathrm{Q}\left(h_{1}, h_{2}\right)$ is also symmetric and linear in each set of variable thus

$$
\begin{aligned}
\widetilde{\mathrm{Q}}\left(\left(h_{1}, h_{2}\right),\left(h_{1}^{\prime}, h_{2}^{\prime}\right)\right) & =\mathrm{Q}\left(h_{1}+h_{1}^{\prime}, h_{2}+h_{2}^{\prime}\right)-\mathrm{Q}\left(h_{1}, h_{2}\right)-\mathrm{Q}\left(h_{1}^{\prime}, h_{2}^{\prime}\right) \\
& =\mathrm{Q}\left(h_{1}, h_{2}^{\prime}\right)+\mathrm{Q}\left(h_{2}, h_{1}^{\prime}\right)
\end{aligned}
$$

then from (40), we get

$$
\widetilde{\mathrm{Q}}_{D}\left(\ell \vec{\alpha}, \lambda_{i}\right)=\sum_{1 \leq k, s \leq m+n} \mathrm{lk}_{k s}\left(\mathrm{Q}\left(\ell \bar{\alpha}_{k}, \delta_{i}^{s} \lambda\right)+\mathrm{Q}\left(\ell \bar{\alpha}_{s}, \delta_{i}^{k} \lambda\right)\right)
$$

and

$$
\ell \bar{L}_{D}\left(\lambda_{i}\right)=\sum_{1 \leq k \leq m+n<s \leq m+n+n_{D_{b l}}}\left(\mathrm{lk}_{k s}+\mathrm{lk}_{s k}\right) \mathrm{Q}\left(\ell \bar{\beta}_{s}, \delta_{i}^{k} \lambda\right) .
$$

Let $X_{D}=\widetilde{\mathrm{Q}}_{D}\left(\ell \vec{\alpha}, \lambda_{i}\right)+\ell \bar{L}_{D}\left(\lambda_{i}\right)$. As $\bar{\beta}_{s}=\omega\left(m_{s}\right)$, we have

$$
\begin{aligned}
X_{D} & =\sum_{1 \leq k \leq m+n} \mathrm{lk}_{k i} \mathrm{Q}\left(\ell \bar{\alpha}_{k}, \lambda_{i}\right)+\mathrm{lk}_{i k} \mathrm{Q}\left(\ell \bar{\alpha}_{k}, \lambda_{i}\right) \\
& +\sum_{m+n<s \leq m+n+n_{D_{b l}}}\left(\mathrm{lk}_{i s}+\mathrm{lk}_{s i}\right) \mathrm{Q}\left(\ell \bar{\alpha}_{s}, \lambda_{i}\right) \\
& =\mathrm{Q}\left(\sum_{k=1}^{m+n+n_{D_{b l}}} \mathrm{lk}_{i k} \omega\left(m_{k}\right)+\sum_{k=1}^{m+n+n_{D_{b l}}} \mathrm{lk}_{k i} \omega\left(m_{k}\right), \ell \lambda_{i}\right) .
\end{aligned}
$$

In addition, for any $1 \leq i \leq m$, in homology, $\sum_{k=1}^{m+n+n_{D_{b l}}} \mathrm{lk}_{i k}\left[m_{k}\right]=$ $\sum_{k=1}^{m+n+n_{D_{b l}}} \mathrm{lk}_{k i}\left[m_{k}\right]$ is the homology class of the parallel of the $i^{\text {th }}$ red 
closed component of $L \cup \Gamma$. So we have

$$
\sum_{k=1}^{m+n+n_{D_{b l}}} \mathrm{lk}_{i k} \omega\left(m_{k}\right)=\sum_{k=1}^{m+n+n_{D_{b l}}} \mathrm{lk}_{k i} \omega\left(m_{k}\right)=\omega\left(\ell_{i}\right) .
$$

It implies that $X_{D}=2 \ell \mathrm{Q}\left(\omega\left(\ell_{i}\right), \lambda_{i}\right)$. Thus with $\widetilde{\mathrm{Q}}_{D}^{c}\left(\vec{\alpha}, \lambda_{i}\right)+\bar{L}_{D}^{c}\left(\lambda_{i}\right)=$ $\sum_{i} f_{i}^{c}\left(h_{i}\right)$, we get

$$
\begin{aligned}
\bar{X} & =\ell\left(2 \mathrm{Q}\left(\omega\left(\ell_{i}\right), \lambda_{i}\right)+\sum_{j} f_{j}^{c}\left(\lambda_{i}\right)\right) \\
& =\ell\left(2 \mathrm{Q}\left(\omega\left(\ell_{i}\right), \lambda_{i}\right)+f_{i}^{c}\left(\lambda_{i}\right)\right) \bmod \ell \mathbb{Z} .
\end{aligned}
$$

By assumption $(M, \Gamma, \omega)$ is compatible triple, i.e.,

$$
2 \mathrm{Q}\left(\omega\left(\ell_{i}\right), h_{i}\right)+f_{i}^{c}\left(h_{i}\right)=0 \text { modulo } \Lambda^{*},
$$

one gets $\bar{X}=0 \bmod \ell \mathbb{Z}$. It implies that

$$
\xi^{P_{D}\left(\alpha+\ell \lambda_{i}\right)}=\xi^{P_{D}(\alpha)}
$$

Hence one concludes $\xi^{P_{D}}$ is $\ell$-periodic on $\Lambda_{\vec{\alpha}}$ in the variables $H_{i, j}$ associated to the close red components.

Lemma 6.3 says that with the cohomology class $\omega$ then the entire function $\xi^{P_{D}} u$ is $\ell$-periodic in the first $r m$ variables on $\Lambda_{\vec{\alpha}}$, where $J_{D}^{b}=\xi^{P_{D}} \sum_{i} u_{i} \otimes f_{i}$. Let

$$
J_{D, \omega}^{b}=\sum_{i} \pi\left(\xi^{P_{D}} u_{i}\right) \otimes f_{i}
$$

then Proposition 4.7 implies that

$$
J_{D, \omega}^{b} \in \mathcal{U}_{\bar{\alpha}_{1}} \otimes \cdots \otimes \mathcal{U}_{\bar{\alpha}_{m}} \otimes \mathcal{L} \mathcal{U}_{\bar{\alpha}_{m+1}} \otimes \cdots \otimes \mathcal{L U}_{\bar{\alpha}_{m+n}} \otimes \operatorname{Hom}_{\mathbb{C}}\left(V, V^{\prime}\right)
$$

where $\pi$ is the projection $\left(\mathcal{U}^{H}\right)^{\widehat{\otimes} m+n} \rightarrow\left(\mathcal{U}^{H}\right)^{\widehat{\otimes} m+n} / I_{\vec{\alpha}}^{H}$.

Proposition 6.4. Let $\Gamma$ be a n-string link graph with $m$ red closed components in $\mathbb{R}^{2} \times[0,1]$ equipped with a compatible cohomology class $\omega$. Let $\vec{\alpha}=\left(\bar{\alpha}_{1}, \ldots, \bar{\alpha}_{m+n}\right)$ be the values of $\omega$ on the meridians of the red components. Let

$$
\begin{aligned}
F_{\mu}(\Gamma, \omega) & =\left(\mu_{\bar{\alpha}_{1}} \otimes \cdots \otimes \mu_{\bar{\alpha}_{m}} \otimes \operatorname{Id}\right)\left(J_{\Gamma, \omega}^{b}\right) \\
& \in \mathcal{L} \mathcal{U}_{\bar{\alpha}_{m+1}} \otimes \cdots \otimes \mathcal{L U}_{\bar{\alpha}_{m+n}} \otimes \operatorname{Hom}_{\mathbb{C}}\left(V, V^{\prime}\right) .
\end{aligned}
$$

Then $F_{\mu}(\Gamma, \omega)$ does not depend on the base points, and is invariant by Kirby 0 and Kirby II moves.

Proof. Let us call $L$ the sub-link of $\Gamma$ formed by the $m$ red closed components. If one moves the base point of the $i^{\text {th }}$ component of $L$ then the universal invariant $J_{\Gamma}^{b}$ only change by terms which have a commutator $c_{i}$ at the $i^{\text {th }}$ factor. If such a term has non zero weight, then so will be its representant $\bar{c}_{i} \in \mathcal{U}_{\bar{\alpha}_{i}}$ and then $\mu_{\bar{\alpha}_{i}}\left(\bar{c}_{i}\right)=0$ by 


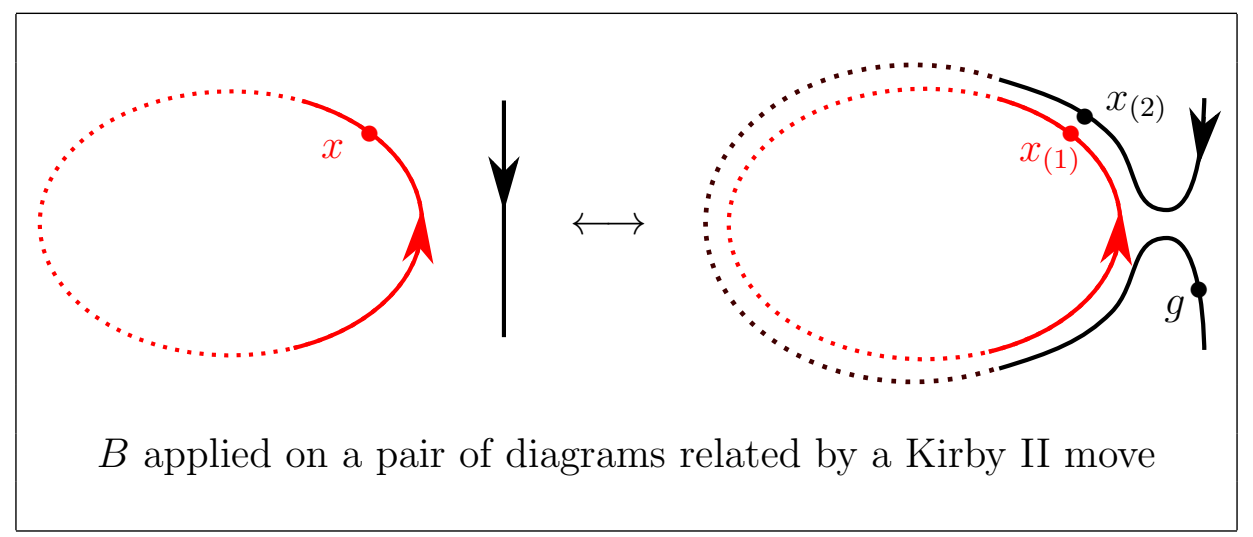

FiguRE 9. Invariance by Kirby II move

Equation (32). Now if the weight of $\bar{c}_{i}$ is zero, it can be written as the commutator of two elements in $\mathcal{L U}_{\bar{\alpha}_{i}}$. From the proof of Lemma 5.2 we see that $\bar{c}_{i}$ belongs to $\left[\mathcal{U}_{\bar{\alpha}_{i}}, \mathcal{L U}_{\bar{\alpha}_{i}}\right] \cap \mathcal{U}_{\bar{\alpha}_{i}}=\left[\mathcal{U}_{\bar{\alpha}_{i}}, \mathcal{U}_{\bar{\alpha}_{i}}\right]$. Then, from Equation $(30)$ we see that $\mu_{\bar{\alpha}_{i}}\left(\bar{c}_{i}\right)=0$. Hence $F_{\mu}(\Gamma, \omega)$ does not depend of the base points.

We now prove $F_{\mu}(\Gamma, \omega)$ does not change under orientation reversal of components of $L$ (i.e., the Kirby 0 move). First, by Lemma 5.4, changing the orientation of a component of $L$ changes $J_{\Gamma}^{b}$ by applying an antipode on the factor corresponding to the component, so the property of $G$-trace in Equation (31) imply $F_{\mu}(\Gamma, \omega)$ does not depend on the orientation.

Finally, to see the Kirby II move hold, we consider a red component $K \subset L$ with beads with product $x \in \widehat{\mathcal{U}^{H}}$ and a blue or red vertical edge $e$ with locally no bead or equivalently a bead 1 , as in the first component of Figure 9. Here the base point of $K$ is assumed to be near the vertical edge and the orientation of $K$ as in the figure. Let $\left(\omega^{\prime}, \vec{\alpha}^{\prime}\right)$ be the image of $(\omega, \vec{\alpha})$ by the second Kirby move. After the move, a pivotal element $g$ has appeared on the slidding edge $e$ and since the two curves are parallel with the same orientation, all the elements along the component $K$ are replaced by $x_{(1)}$ on $K$ and $x_{(2)}$ on $e$ where $\Delta x=x_{(1)} \otimes x_{(2)}$. Since modulo $I_{\vec{\alpha}}, x \in \mathcal{U}_{\bar{\beta}}$ we have that modulo $I_{\vec{\alpha}^{\prime}}$, $\Delta x \in \mathcal{U}_{\bar{\beta}-\bar{\alpha}} \otimes \mathcal{U}_{\bar{\alpha}}$. Then (28) implies that the values of $F_{\mu}$ before and after the Kirby II move are the same.

Up to isotopy, one can identify closed bichrome graphs in $\mathbb{R}^{2} \times[0,1]$ with closed bichrome graphs in $S^{3}$. The same holds for pair $(\Gamma, \omega)$ where $\omega$ is a compatible cohomology class in the complement of the smoothing $\widetilde{\Gamma}$ of a closed bichrome graph $\Gamma$. Hence Proposition 6.4 induce a complex valued invariant of compatible triple $\left(S^{3}, \Gamma, \omega\right)$ that we still denote by $F_{\mu}(\Gamma, \omega) \in \mathbb{C}$. 
Theorem 6.5 (Graded Hennings invariant). Let $(M, \Gamma, \omega)$ be compatible triple where $M$ is a oriented closed 3-manifolds. Let $L \subset S^{3}$ be a framed link which is a surgery presentation of $M$. Denote $\omega$ as the restriction of the cohomology class $\omega$ in $H^{1}\left(S^{3} \backslash(\widetilde{\Gamma} \cup L), G\right)$. Define

$$
\mathrm{H}(M, \Gamma, \omega)=\delta^{-s} F_{\mu}(L \cup \Gamma, \omega)
$$

where $\delta=\mu_{\overline{0}}\left(g_{\overline{0}}^{-1} \theta_{\overline{0}}\right)$ and $s$ is the signature of the linking matrix of $L$. Then $\mathrm{H}$ is a well defined invariant of diffeomorphism class of $(M, \Gamma, \omega)$.

Proof. From Proposition 6.1, we have $\left(S^{3}, \Gamma \cup L, \omega\right)$ is a compatible triple and by Theorem 6.2 any two such presentation are related by Kirby moves. By Proposition $6.4, F_{\mu}(L \cup \Gamma, \omega)$ is invariant by Kirby 0 and Kirby II moves. For the Kirby I move, if we add a \pm 1 -framed unknot to $L$ with meridian $m_{0}$, then $\omega\left(m_{0}\right)=\overline{0}$ because the class of $m_{0}$ is zero in $H_{1}(M \backslash \widetilde{\Gamma}, \mathbb{Z})$. Then the invariant $F^{\prime}$ is multiplied by $\lambda_{\overline{0}}\left(\theta_{\overline{0}}\right)^{\mp 1}=\delta^{\mp 1}$. At the same time the signature of the linking matrix $s$ changes by \pm 1 , so $\mathrm{H}(M, \Gamma, \omega)$ does not change under the Kirby I move.

In many known examples the invariant $\mathrm{H}$ is zero for "generic" compatible triples. In particular, in the case of Example 2.3, the invariant $\mathrm{H}$ is zero when $\Gamma$ is colored with a projective module or when $\omega$ has non-integral values. However, as we will now explain, m-traces can be used to renormalize $\mathrm{H}$ to define a non-zero invariant for these "generic" compatible triples.

Proposition 6.6. Let $\Gamma$ be a n-string link graph and $\left(\mathbb{R}^{2} \times[0,1], \Gamma, \omega\right)$ be a compatible triple, then $F_{\mu}(\Gamma, \omega) \in \mathcal{L U}_{\bar{\alpha}_{1}} \otimes \cdots \otimes \mathcal{L U}_{\bar{\alpha}_{n}} \otimes \operatorname{Hom}_{\mathbb{C}}\left(V, V^{\prime}\right)$ is equivariant: write $F_{\mu}(\Gamma, \omega)=\sum_{i} x_{i} \otimes f_{i}$ then let $\mathcal{U}_{\otimes \vec{\alpha}}^{H}=\mathcal{U}_{\bar{\alpha}_{1}}^{H} \widehat{\otimes} \cdots \widehat{\otimes} \mathcal{U}_{\bar{\alpha}_{n}}^{H}$ where the factors are equipped with the action of $\widehat{\mathcal{U}^{H}}$ by left multiplication. Then

$$
\sum_{i} L_{x_{i}} \otimes f_{i} \in \operatorname{Hom}_{\mathbb{C}}\left(\mathcal{U}_{\otimes \vec{\alpha}}^{H} \otimes V, \mathcal{U}_{\otimes \vec{\alpha}}^{H} \otimes V^{\prime}\right)
$$

is a morphism of $\widehat{\mathcal{U}^{H}}$-modules.

Proof. The proof is by induction on the number $m$ of closed red components of $\Gamma$. If $m=0$, by Equation (35) of Theorem 5.1, the universal invariant $J(\check{\Gamma})$ is $\widehat{\mathcal{U}}^{H}$-equivariant. Since the projection modulo the ideals $I_{\bar{\alpha}}$ is equivariant, then $F_{\mu}(\Gamma, \omega)$ is $\widehat{\mathcal{U}^{H}}$-equivariant.

Now assume $m>0$ and consider a $(n+1)$-string link graph $\check{\Gamma}$ whose left braid closure of the first strand produces $\Gamma$. Let $\check{D}$ be a diagram of $\check{\Gamma}$ and $D$ its left braid closure obtained by joining the $m$ top endpoints of $\check{D}$ to its $m$ bottom endpoints using an arc disjoint from the diagram on its left. We still denotes by $\omega$ the restriction of the cohomology class to $\mathbb{R}^{2} \times[0,1] \backslash \check{\Gamma} \subset \mathbb{R}^{2} \times[0,1] \backslash \Gamma$. Then by induction $F_{\mu}(\check{\Gamma}, \omega)$ is $\widehat{\mathcal{U}}_{-}$ equivariant. Now computing the invariants using $\check{D}$ and $D$ with the 
new base point on the left arc, we have that if $F_{\mu}(\check{\Gamma}, \omega)=\sum_{i} y_{i} \otimes z_{i} \otimes f_{i}$ with $y_{i} \in \mathcal{U}_{\bar{\alpha}}$ then

$$
F_{\mu}(\Gamma, \omega)=\sum_{i} \mu_{\bar{\alpha}}\left(y_{i} g^{-1}\right) z_{i} \otimes f_{i} .
$$

Now for $u \in \mathcal{U}$, let $\Delta^{(n+2)} u=u_{a} \otimes u_{b} \otimes u_{c}$ with $u_{a} \in \mathcal{U}, u_{b} \in \mathcal{U}^{\otimes n}$ and $u_{c} \in \mathcal{U}$. We can write $\Delta^{n+1} u=\varepsilon\left(u_{a}\right) u_{b} \otimes u_{c}$ and then

$$
\begin{aligned}
F_{\mu}(\Gamma, \omega)\left(\Delta^{n+1} u\right) & =\sum_{i} \mu_{\bar{\alpha}}\left(y_{i} \varepsilon\left(u_{a}\right) g^{-1}\right) z_{i} u_{b} \otimes f_{i}\left(u_{c} \cdot\right) \\
& =\sum_{i} \mu_{\bar{\alpha}}\left(y_{i} u_{a(2)} S^{-1}\left(u_{a(1)}\right) g^{-1}\right) z_{i} u_{b} \otimes f_{i}\left(u_{c} \cdot\right) \\
& =\sum_{i} \mu_{\bar{\alpha}}\left(u_{a(2)} y_{i} g^{-1} S\left(u_{a(1)}\right)\right) u_{b} z_{i} \otimes u_{c} f_{i} \\
& =\sum_{i} \mu_{\bar{\alpha}}\left(S\left(u_{a(1)}\right) u_{a(2)} y_{i} g^{-1}\right) u_{b} z_{i} \otimes u_{c} f_{i} \\
& =\sum_{i} \varepsilon\left(u_{a}\right) \mu_{\bar{\alpha}}\left(y_{i} g^{-1}\right) u_{b} z_{i} \otimes u_{c} f_{i}=\left(\Delta^{n+1} u\right) F_{\mu}(\Gamma, \omega),
\end{aligned}
$$

where the third equality uses the equivariance of $F_{\mu}(\check{\Gamma}, \omega)$ and the cyclicity of the symmetrised integral (30). For the $\mathfrak{H}$-linearity, we need to use Equation (32). By this equality only the terms of (44) where $y_{i}$ commute with any element $H$ of $\mathfrak{H}$ will contribute to the sum. But since $H$ is primitive, $\Delta^{(n+2)} H=H \otimes 1+1 \otimes \Delta^{(n+1)} H$ so projecting Equation (44) on weight zero space for the first factor, we have

$$
\sum_{\left|y_{i}\right|=0} y_{i} \otimes\left(\left(z_{i} \otimes f_{i}\right) \Delta^{(n+1)} H\right)=\sum_{\left|y_{i}\right|=0} y_{i} \otimes\left(\Delta^{(n+1)} H\left(z_{i} \otimes f_{i}\right)\right)
$$

which implies the $H$ linearity of $F_{\mu}(\Gamma, \omega)$.

Definition 6.7. The compatible triple $(M, \Gamma, \omega)$ is called a graphadmissible triple if there exists a blue edge of $\Gamma$ colored by $V \in \operatorname{Proj}\left(\mathscr{C}^{H}\right)$.

Let $\left(S^{3}, \Gamma, \omega\right)$ be a graph-admissible triple with $\Gamma$ is a closed bichrome graph embedded in $S^{3}$. A cutting presentation of $\Gamma$ is a bichrome graph $\Gamma_{V} \in \mathbb{R}^{2} \times[0,1]$ with bottom and top end point $(V,+)$ whose braid closure is isotopic to $\left(S^{3}, \Gamma\right)$. It is equipped with the induced cohomology class still denoted $\omega$. Then Proposition 6.6 implies that $F_{\mu}\left(\Gamma_{V}, \omega\right) \in \operatorname{Hom}_{\mathbb{C}}(V, V)$ is a morphism of $\widehat{\mathcal{U}^{H}}$-modules (as $n=0$ ). But $V$ is finite dimensional and so $F_{\mu}\left(\Gamma_{V}, \omega\right)$ is a morphism of $\mathcal{U}^{H_{-}}$ modules, i.e. $F_{\mu}\left(\Gamma_{V}, \omega\right) \in \operatorname{End}_{\mathscr{C}^{H}}(V)$. Let

$$
F_{\mu}^{\prime}(\Gamma, \omega)=\mathrm{t}_{V}\left(F_{\mu}\left(\Gamma_{V}, \omega\right)\right) .
$$

Proposition 6.8. The modified invariant $F_{\mu}^{\prime}(\Gamma, \omega)$ of Equation (45) does not depend on the choice of a cutting presentation $\Gamma_{V}$. Furthermore, it is invariant by Kirby 0 and Kirby II move and for any closed 
compatible triple $\left(S^{3}, \Gamma^{\prime}, \omega^{\prime}\right)$, the modified invariant of the disjoint union is given by

$$
F_{\mu}^{\prime}\left(\Gamma \sqcup \Gamma^{\prime}, \omega^{\prime \prime}\right)=F_{\mu}^{\prime}(\Gamma, \omega) F_{\mu}\left(\Gamma^{\prime}, \omega^{\prime}\right)
$$

where $\omega^{\prime \prime}$ is the cohomology class induced by $\omega$ and $\omega^{\prime}$.

Proof. The proof of the first statement is similar to that in [14]: indeed, if $\Gamma_{V}$ and $\Gamma_{V^{\prime}}$ are both cutting presentation of $\Gamma$ then there exists $\Gamma_{V, V^{\prime}}$ such that $\Gamma_{V}$ and $\Gamma_{V^{\prime}}$ are the partial braid closure of $\Gamma_{V, V^{\prime}}$, and $\Gamma_{V, V^{\prime}}$ conjugated by the braiding, respectively. Then we have

$$
\begin{aligned}
\mathrm{t}_{V}\left(F_{\mu}\left(\Gamma_{V}, \omega\right)\right) & =\mathrm{t}_{V}\left(\operatorname{ptr}_{V^{\prime}}\left(F_{\mu}\left(\Gamma_{V, V^{\prime}}, \omega\right)\right)\right) \\
& =\mathrm{t}_{V \otimes V^{\prime}}\left(F_{\mu}\left(\Gamma_{V, V^{\prime}}, \omega\right)\right) \\
& =\mathrm{t}_{V^{\prime} \otimes V}\left(c_{V^{\prime}, V^{-}}^{-1} F_{\mu}\left(\Gamma_{V, V^{\prime}}, \omega\right) c_{V^{\prime}, V}\right) \\
& =\mathrm{t}_{V^{\prime}}\left(\operatorname{ptr}_{V}\left(c_{V^{\prime}, V}^{-1} F_{\mu}\left(\Gamma_{V, V^{\prime}}, \omega\right) c_{V^{\prime}, V}\right)\right) \\
& =\mathrm{t}_{V^{\prime}}\left(F_{\mu}\left(\Gamma_{V^{\prime}}, \omega\right)\right)
\end{aligned}
$$

where the second and fourth equality (resp. third equality) follow from the partial trace property (resp. cyclicity) of the m-trace. Now the invariance by Kirby 0 and 2 moves of $F_{\mu}^{\prime}(\Gamma, \omega)$ follows from that of $F_{\mu}\left(\Gamma_{V}, \omega\right)$. Finally for a disjoint union, just remark that is $\Gamma_{V}$ is a cutting presentation of $\Gamma$, then $\Gamma_{V} \sqcup \Gamma^{\prime}$ is a cutting presentation of $\Gamma \sqcup \Gamma^{\prime}$ and $F_{\mu}\left(\Gamma_{V} \sqcup \Gamma^{\prime}, \omega^{\prime \prime}\right)=F_{\mu}\left(\Gamma_{V}, \omega\right) F_{\mu}\left(\Gamma^{\prime}, \omega^{\prime}\right)$.

Then the modified invariant naturally extends to graph-admissible triples $(M, \Gamma, \omega)$ with $\Gamma$ a closed bichrome graph embedded in $M$ :

Theorem 6.9 (Modified graded Hennings invariant). Let $M$ be an oriented closed 3-manifold and $(M, \Gamma, \omega)$ be a graph-admissible compatible triple with surgery presentation $\left(S^{3}, \Gamma \cup L, \omega\right)$. Define

$$
\mathrm{H}^{\prime}(M, \Gamma, \omega)=\delta^{-s} F_{\mu}^{\prime}(L \cup \Gamma, \omega)
$$

where $\delta=\mu_{\overline{0}}\left(g_{\overline{0}}^{-1} \theta_{\overline{0}}\right)$ and $s$ is the signature of the linking matrix of $L$. Then $\mathrm{H}^{\prime}$ is a well defined invariant of diffeomorphism class of $(M, \Gamma, \omega)$. Furthermore, if $\left(M^{\prime}, \Gamma^{\prime}, \omega^{\prime}\right)$ is any closed compatible triple, the modified invariant of the connected sum is

$$
\mathrm{H}^{\prime}\left(M \# M^{\prime}, \Gamma \sqcup \Gamma^{\prime}, \omega^{\prime \prime}\right)=\mathrm{H}^{\prime}(M, \Gamma, \omega) \mathrm{H}\left(M^{\prime}, \Gamma^{\prime}, \omega^{\prime}\right)
$$

where $\omega^{\prime \prime}$ is the cohomology class induced by $\omega$ and $\omega^{\prime}$.

Proof. From Theorem 6.2 it is enough to show that $\mathrm{H}^{\prime}$ is invariant under the Kirby moves. Proposition 6.8 imply the Kirby 0 and Kirby II hold. As in the proof of Theorem 6.5 , if we add a \pm 1 -framed unknot to $L$ with meridian $m_{0}$, then $\omega\left(m_{0}\right)=\overline{0}$ and the invariant $F^{\prime}$ is multiplied by $\lambda_{\overline{0}}\left(\theta_{\overline{0}}\right)^{\mp 1}=\delta^{\mp 1}$. So the Kirby I move holds. The last property of the theorem, follows from considering a surgery presentation of the connected sum, which is a disjoint union of surgery presentations in $S^{3}$. 


\section{MODIFIED SYMMETRIZED INTEGRAL}

We introduce the notion of a modified integral which allows us to relax the admissibility condition for the modified graded Hennings invariant and in particular gives an invariant for empty 3-manifolds.

Given $\bar{\alpha}_{i} \in G$, let $\vec{\alpha}=\left(\bar{\alpha}_{1}, \ldots, \bar{\alpha}_{n}\right), \bar{\beta}_{j}=\sum_{i=j}^{n} \bar{\alpha}_{i}$ for $j \leq n$ and $\bar{\beta}=\bar{\beta}_{1}$. Denote $\mathcal{U}_{\otimes \vec{\alpha}}=\mathcal{U}_{\bar{\alpha}_{1}} \otimes \mathcal{U}_{\bar{\alpha}_{2}} \otimes \ldots \otimes \mathcal{U}_{\bar{\alpha}_{n}}$ and let $\Delta_{\vec{\alpha}}: \mathcal{U}_{\bar{\beta}} \rightarrow \mathcal{U}_{\otimes \vec{\alpha}}$ be the map

$$
\Delta_{\vec{\alpha}}=\left(1 \otimes \ldots \otimes 1 \otimes \Delta_{\bar{\alpha}_{n-1}, \bar{\beta}_{n}}\right) \ldots\left(1 \otimes \Delta_{\bar{\alpha}_{2}, \bar{\beta}_{3}}\right) \Delta_{\bar{\alpha}_{1}, \bar{\beta}_{2}} .
$$

By definition of a Hopf $G$-coalgebra $\Delta_{\vec{\alpha}}$ is an algebra morphism. We denote by $Z_{\vec{\alpha}}$ the centralizer of $\Delta_{\vec{\alpha}}\left(\mathcal{U}_{\bar{\beta}}\right)$ which is a subalgebra of $\mathcal{U}_{\otimes} \vec{\alpha}$ formed by elements which commute with any element of $\Delta_{\vec{\alpha}}\left(\mathcal{U}_{\bar{\beta}}\right)$. In particular, for $n=1$ and $\vec{\alpha}=(\bar{\alpha})$ then $Z_{\bar{\alpha}}$ is the center of $\mathcal{U}_{\bar{\alpha}}$.

Lemma 7.1. Recall $L_{g}$ is the left multiplication of $g$. We have

$$
\left(\mu_{\bar{\alpha}} L_{g_{\bar{\alpha}}^{-1}} \otimes \operatorname{Id}\right)\left(Z_{(\bar{\alpha}, \bar{\beta})}\right) \subset Z_{\bar{\beta}} \text { and }\left(\operatorname{Id} \otimes \mu_{\bar{\beta}} L_{g_{\bar{\beta}}}\right)\left(Z_{(\bar{\alpha}, \bar{\beta})}\right) \subset Z_{\bar{\alpha}} .
$$

Proof. Let $z=\sum z_{1} \otimes z_{2} \in Z_{(\bar{\alpha}, \bar{\beta})}, u \in \mathcal{U}_{\bar{\beta}}$ and let $\Delta_{(-\bar{\alpha}, \bar{\alpha}, \bar{\beta})}(u)=$ $u_{(1)} \otimes u_{(2)} \otimes u_{(3)}$. Then $\sum u_{(1)} \otimes z_{1} u_{(2)} \otimes z_{2} u_{(3)}=\sum u_{(1)} \otimes u_{(2)} z_{1} \otimes u_{(3)} z_{2}$ by definition of $Z_{(\bar{\alpha}, \bar{\beta})}$ so

$$
\begin{gathered}
\sum \mu_{\bar{\alpha}}\left(g_{\bar{\alpha}}^{-1} z_{1}\right) z_{2} u=\sum \mu_{\bar{\alpha}}\left(g_{\bar{\alpha}}^{-1} z_{1} u_{(2)} S_{-\bar{\alpha}}^{-1}\left(u_{(1)}\right)\right) z_{2} u_{(3)} \\
=\sum \mu_{\bar{\alpha}}\left(g_{\bar{\alpha}}^{-1} S_{-\bar{\alpha}}\left(u_{(1)}\right) z_{1} u_{(2)}\right) \otimes z_{2} u_{(3)}= \\
\sum \mu_{\bar{\alpha}}\left(g_{\bar{\alpha}}^{-1} S_{-\bar{\alpha}}\left(u_{(1)}\right) u_{(2)} z_{1}\right) \otimes u_{(3)} z_{2}=\sum \mu_{\bar{\alpha}}\left(g_{\bar{\alpha}}^{-1} z_{1}\right) u z_{2} .
\end{gathered}
$$

The proof is similar for the second inclusion.

Definition 7.2. Let $X$ be a subset of $G$. We say that a family of maps $\left\{\mu_{\bar{\alpha}}^{\prime}: Z_{\bar{\alpha}} \rightarrow \mathbb{C}\right\}_{\bar{\alpha} \in G \backslash X}$ is a modified integral on $G \backslash X$ if for any $\bar{\alpha}, \bar{\beta} \in G \backslash X$ the following two linear forms are equal on $Z_{(\bar{\alpha}, \bar{\beta})}$ :

$$
\mu_{\bar{\alpha}} L_{g_{\bar{\alpha}}^{-1}} \otimes \mu_{\bar{\beta}}^{\prime}=\mu_{\bar{\alpha}}^{\prime} \otimes \mu_{\bar{\beta}} L_{g_{\bar{\beta}}} .
$$

Theorem 7.3. Let $X$ be the subset of $G$ such that $G \backslash X$ is the set of $\bar{\alpha}$ where $\mathcal{U}_{\bar{\alpha}}$ is semi-simple. Then there exists a family of central elements $\left\{z_{\bar{\alpha}} \in Z_{\bar{\alpha}}\right\}_{\bar{\alpha} \in G \backslash X}$ such that $\mu_{\bar{\alpha}}(x)=\operatorname{tr}_{\mathcal{U}_{\bar{\alpha}}}^{\mathbb{C}}\left(L_{z_{\bar{\alpha}}} L_{x}\right)$ for all $x \in$ $\mathcal{U}_{\bar{\alpha}}$. Furthermore, there exists a modified integral on $G \backslash X$ defined by

$$
\mu_{\bar{\alpha}}^{\prime}(z):=\mu_{\bar{\alpha}}\left(z_{\bar{\alpha}} z\right)=\operatorname{tr}_{\mathcal{U}_{\bar{\alpha}}}^{\mathbb{C}}\left(L_{z_{\bar{\alpha}}^{2} z}\right)
$$

for all $z \in Z_{\bar{\alpha}}$.

Proof. Since $\mathcal{U}_{\bar{\alpha}}$ is a finite-dimensional semi-simple algebra for $\bar{\alpha} \in$ $G \backslash X$ then it is isomorphic to a product of matrix algebras: $\mathcal{U}_{\bar{\alpha}} \cong$ $\bigoplus_{i} \operatorname{Mat}\left(V_{i}\right)$ where $V_{i}=\mathbb{C}^{n_{i}}$ are its irreducible representations. Then the identity matrices of each summand form a basis $\left\{z_{i}\right\}_{i}$ of the center $Z_{\bar{\alpha}}$ and the characters $x \mapsto \operatorname{tr}_{\mathcal{U}_{\bar{\alpha}}}^{\mathbb{C}}\left(L_{z_{i}} L_{x}\right)=n_{i} \operatorname{tr}_{V_{i}}^{\mathbb{C}}\left(\rho_{V_{i}}(x)\right)$ form a basis of $\mathrm{HH}_{0}\left(\mathcal{U}_{\bar{\alpha}}\right)^{*}:=\left(\mathcal{U}_{\bar{\alpha}} /\left[\mathcal{U}_{\bar{\alpha}}, \mathcal{U}_{\bar{\alpha}}\right]\right)^{*}$. Finally since $\mu_{\bar{\alpha}} \in \mathrm{HH}_{0}\left(\mathcal{U}_{\bar{\alpha}}\right)^{*}$ there exists 
$\left\{\delta_{i} \in \mathbb{C}\right\}_{i}$ such that $\mu_{\bar{\alpha}}(x)=\sum_{i} \delta_{i} \operatorname{tr}_{\mathcal{U}_{\bar{\alpha}}}^{\mathbb{C}}\left(L_{z_{i}} L_{x}\right)$ for all $x \in \mathcal{U}_{\bar{\alpha}}$. We define $z_{\bar{\alpha}}=\sum_{i} \delta_{i} z_{i}$.

Remark that since $z_{i} z_{j}=\delta_{i}^{j} z_{i}$, applying the above formula for $x=z_{i}$ gives $\mu_{\bar{\alpha}}\left(z_{i}\right)=\operatorname{tr}_{\mathcal{U}_{\bar{\alpha}}}^{\mathbb{C}}\left(L_{z_{i}}\right) \delta_{i}=n_{i}^{2} \delta_{i}$ because $L_{z_{i}}$ is the identity of the block $\operatorname{Mat}\left(V_{i}\right)$. But the symmetrized integral of $z_{i}$ is the modified trace of the induced endomorphism of $\mathcal{U}_{\bar{\alpha}}$ (by left or right multiplication) which is a projector on $V_{i}^{\oplus n_{i}}$ then $\mu_{\bar{\alpha}}\left(z_{i}\right)=n_{i} d_{i}$ where $d_{i}$ is the modified dimension of $V_{i}$ (see [18]), thus $\delta_{i}=\frac{d_{i}}{n_{i}}$. Finally, one gets

$$
\mu_{\bar{\alpha}}=\sum_{i} d_{i} \operatorname{tr}_{V_{i}}^{\mathbb{C}} \circ \rho_{V_{i}}
$$

Let us now prove that $\mu_{\bar{\alpha}}^{\prime}=\mu_{\bar{\alpha}}\left(z_{\bar{\alpha}} \cdot\right)$ is a modified integral. Let $x \in Z_{\bar{\alpha}, \bar{\beta}}$ and consider the endomorphism $f$ of $\mathcal{U}_{\bar{\alpha}} \otimes \mathcal{U}_{\bar{\beta}}$ given by left multiplication by $\left(z_{\bar{\alpha}} \otimes z_{\bar{\beta}}\right) x$. Then the properties of the partial trace imply that

$$
\operatorname{t}_{\mathcal{U}_{\bar{\alpha}}}\left(\operatorname{ptr}_{\mathcal{U}_{\bar{\beta}}}(f)\right)=\mathrm{t}_{\mathcal{U}_{\bar{\beta}}}\left(\operatorname{ptr}_{\mathcal{U}_{\bar{\alpha}}}(f)\right)
$$

now the partial trace in the category is the partial trace in Vect twisted by the action of the pivotal elements and the modified trace on $\mathcal{U}_{\bar{\alpha}}$ is given by $\mathrm{t}_{\mathcal{U}_{\bar{\alpha}}}=\mu_{\bar{\alpha}}=\operatorname{tr}_{\mathcal{U}_{\bar{\alpha}}}^{\mathbb{C}}\left(L_{z_{\bar{\alpha}}} \cdot\right)$ thus Equation (49) becomes

$$
\operatorname{tr}_{\mathcal{U}_{\bar{\alpha}} \otimes \mathcal{U}_{\bar{\beta}}}^{\mathbb{C}}\left(L_{z_{\bar{\alpha}}^{2} \otimes g_{\bar{\beta}} z_{\bar{\beta}}} L_{x}\right)=\operatorname{tr}_{\mathcal{U}_{\bar{\alpha}} \otimes \mathcal{U}_{\bar{\beta}}}^{\mathbb{C}}\left(L_{z_{\bar{\alpha}} g_{\bar{\alpha}}^{-1} \otimes z_{\bar{\beta}}^{2}} L_{x}\right)
$$

with $\operatorname{tr}_{\mathcal{U}_{\bar{\alpha}} \otimes \mathcal{U}_{\bar{\beta}}}^{\mathbb{C}}=\operatorname{tr}_{\mathcal{U}_{\bar{\alpha}}}^{\mathbb{C}} \otimes \operatorname{tr}_{\mathcal{U}_{\bar{\beta}}}^{\mathbb{C}}$ so $\mu_{\bar{\alpha}}^{\prime} \otimes \mu_{\bar{\beta}}\left(\left(1 \otimes g_{\bar{\beta}}\right) x\right)=\mu_{\bar{\alpha}} \otimes \mu_{\bar{\beta}}^{\prime}\left(\left(g_{\bar{\alpha}}^{-1} \otimes 1\right) x\right)$ which proves that $\mu^{\prime}$ is a modified integral.

Remark that the modified integral $\mu_{\bar{\alpha}}^{\prime}$ of Theorem 7.3 has a natural extension as a linear form on the whole algebra $\mathcal{U}_{\bar{\alpha}}$. We call $\mu_{\bar{\alpha}}^{\prime}$ the canonical modified integral.

Recall the definition of a graph-admissible triple given in Definition 6.7 .

Definition 7.4. A compatible triple $(M, \Gamma, \omega)$ is called a $G$-admissible triple if there exists a red edge of $\Gamma$ colored by $\bar{\alpha} \in G \backslash X$. A triple $(M, \Gamma, \omega)$ is called an admissible triple if $(M, \Gamma, \omega)$ is a $G$-admissible triple or a graph-admissible triple.

As for graph-admissible closed bichrome graph, we can introduce the notion of cutting presentation $\Gamma_{\bar{\alpha}}$ of $G$-admissible triple $\left(S^{3}, \Gamma, \omega\right)$ by cutting a red edge rather than a blue edge: $\Gamma_{\bar{\alpha}}$ is a 1-string link graph whose braid closure is $\Gamma$ and where $\omega$ take on the meridian of the open strand the value $\bar{\alpha} \in G \backslash X$. Remark that the universal invariant of such a graph is $\ell$-periodic in its $r$-variables and equivariant so $F_{\mu}\left(\Gamma_{\bar{\alpha}}\right) \in Z_{\bar{\alpha}}$.

Proposition 7.5. There exists unique extensions of $F_{\mu}^{\prime}$ to $G$-admissible bichrome closed graphs (resp. $\mathrm{H}^{\prime}$ to G-admissible triples)) with

$$
F_{\mu}^{\prime}(\Gamma, \omega)=\mu_{\bar{\alpha}}^{\prime}\left(F_{\mu}\left(\Gamma_{\bar{\alpha}}, \omega\right)\right)
$$




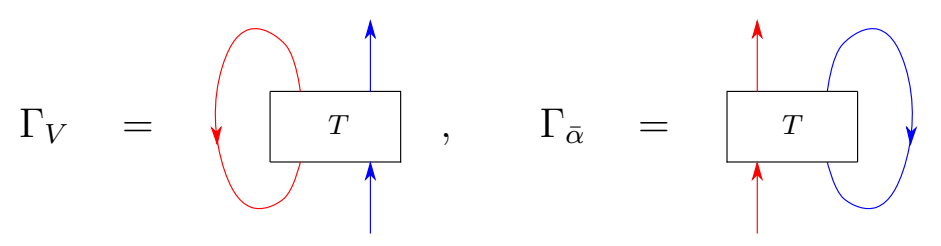

Figure 10. Cutting presentation $\Gamma_{V}$ at blue edge and $\Gamma_{\bar{\alpha}}$ at red edge

where $\Gamma_{\bar{\alpha}}$ is a cutting presentation of $\Gamma$. The extension of $\mathrm{H}^{\prime}$ is given by

$$
\mathrm{H}^{\prime}(M, \Gamma, \omega)=\delta^{-s} F_{\mu}^{\prime}(L \cup \Gamma, \omega)
$$

where $\left(S^{3}, \Gamma \cup L, \omega\right)$ is a surgery presentation of an admissible triple $(M, \Gamma, \omega)$, the constant $\delta$ is $\mu_{\overline{0}}\left(g_{\overline{0}}^{-1} \theta_{\overline{0}}\right)$ and $s$ is the signature of the linking matrix of $L$.

Proof. Suppose that $\Gamma$ admits two $G$-admissible cutting presentations $\Gamma_{\bar{\alpha}}$ and $\Gamma_{\bar{\beta}}$. Then cutting the two edges we get a 2 -string link graph whose image by $F_{\mu}$ is in $Z_{(\alpha, \beta)}$ because of Proposition 6.6. Then Definition 7.2 implies that the invariant computed by $\Gamma_{\bar{\alpha}}$ and $\Gamma_{\bar{\beta}}$ are the same.

Suppose now that $\Gamma$ admits a $G$-admissible cutting presentation $\Gamma_{\bar{\alpha}}$ obtained by cutting a red edge and a graph-admissible cutting presentation $\Gamma_{V}$ for some projective $V \in \mathscr{C}_{\bar{\beta}}^{H}$ obtained by cutting a blue edge. Then cutting the two edges lead to a graph $T$ whose image by $F_{\mu}$ is in $\mathcal{U}_{\bar{\alpha}} \otimes \operatorname{End}_{\mathbb{C}}(V)$ (see Figure 10). Let us write $F_{\mu}(T, \omega)=L_{x} \otimes f$ (we omit the sum). Then

$$
\begin{aligned}
& \mu_{\bar{\alpha}}^{\prime}\left(F_{\mu}\left(\Gamma_{\bar{\alpha}}, \omega\right)\right)=\mu_{\bar{\alpha}}^{\prime}\left(\stackrel{\uparrow}{L_{x} \otimes f} \gamma\right)=\mu_{\bar{\alpha}}^{\prime}(x) \operatorname{tr}_{\mathcal{U}_{\bar{\beta}}}^{\mathbb{C}}\left(\rho_{V}\left(g_{\bar{\beta}}\right) f\right) \\
& =\mu_{\bar{\alpha}}\left(z_{\bar{\alpha}} x\right) \operatorname{tr}_{R}^{\mathscr{C}^{H}}(f)=\operatorname{t}_{\mathcal{U}_{\bar{\alpha}}}\left(L_{z_{\bar{\alpha}}} L_{x}\right) \operatorname{tr}_{\mathcal{U}_{\bar{\beta}}}^{\mathbb{C}}\left(\rho_{V}\left(g_{\bar{\beta}}\right) f\right) \\
& =F_{\mu}^{\prime}\left(\sqrt{\frac{L_{x} \otimes f}{z_{\bar{\alpha}}}} \downarrow\right)=\mathrm{t}_{V}\left(\sqrt[\frac{1}{L_{x} \otimes f}]{z_{\bar{\alpha}} \uparrow}\right) \\
& =\operatorname{tr}_{\mathcal{U}_{\bar{\alpha}}}^{\mathbb{C}}\left(L_{z_{\bar{\alpha}}} L_{g_{\bar{\alpha}}^{-1}} L_{x}\right) \mathrm{t}_{V}(f)=\mu_{\bar{\alpha}}\left(x g_{\bar{\alpha}}^{-1}\right) \mathbf{t}_{V}(f) \\
& =\mathrm{t}_{V}\left(\curlyvee \stackrel{\uparrow}{L_{x} \otimes f}\right)=\mathrm{t}_{V}\left(F_{\mu}\left(\Gamma_{V}, \omega\right)\right)
\end{aligned}
$$


where the third equality follows because $x \operatorname{tr}_{R}^{\mathscr{C}^{H}}(f) \in Z_{\bar{\alpha}}$ is central and so $\operatorname{tr}_{R}^{\mathscr{C}^{H}}(f) L_{z_{\bar{\alpha}} x}=\operatorname{tr}_{R}^{\mathscr{C}^{H}}(f) R_{z_{\bar{\alpha}} x}$ has its modified trace given by Equation (33).

\section{RELATIONS WITH OTHER NON SEMI-SIMPLE INVARIANTS}

We compare the invariant of this paper with three previously defined invariants: (1) the one from the second author with $\mathfrak{s l}(2 \mid 1)$, (2) with the invariant defined by the first and last author with F. Costantino using the weight representations of the unrolled quantum groups and (3) the modified Hennings invariants.

8.1. The $\mathfrak{s l}(2 \mid 1)$ case. Let $\ell \geq 3$ be an odd integer. In [17] the second author considers the semi-restricted super algebra $\mathcal{U}_{\xi} \mathfrak{s l}(2 \mid 1)$ and unrolled super algebra $\mathcal{U}_{\xi}^{H} \mathfrak{s l}(2 \mid 1)$ associated with the super Lie algebra $\mathfrak{s l}(2 \mid 1)$. It is shown that $\widehat{\mathcal{U}_{\xi}^{H}} \mathfrak{s l}(2 \mid 1)$ is a topological ribbon Hopf super algebra and its bosonization $\widehat{\mathcal{U}_{\xi}^{H}} \mathfrak{s l}(2 \mid 1)^{\sigma}=\widehat{\mathcal{U}}_{\xi}^{H} \mathfrak{s l}(2 \mid 1) \rtimes \mathbb{Z} / 2 \mathbb{Z}$ is a topological ribbon Hopf algebra. Consider the bosonization $\mathcal{U}_{\xi} \mathfrak{s l}(2 \mid 1)^{\sigma}$ of the semi-restricted super quantum group. This algebra can be realized as the $\mathcal{U}$ of this paper where $\Lambda$ is the root lattice of $\mathfrak{s l}(2 \mid 1)$ and $W$ is the $\Lambda$-graded vector space generated by the "E" and "F" parts of the Poincaré-Birkhoff-Witt basis. It follows from [17] that $\mathcal{U}$ satisfies the five Axioms 1-5. Moreover, one can identify $\widehat{\mathcal{U}_{\xi}^{H}} \mathfrak{s l}(2 \mid 1)^{\sigma}$ with the unrolled version $\widehat{\mathcal{U}^{H}}$ of $\mathcal{U}$ as defined in Section 3 .

The construction of [17] was the motivation for this paper. It produces an invariant $\mathcal{J}(M, \omega)$ which is equal to the graded Hennings invariant of this paper:

$$
\mathcal{J}(M, \omega)=\mathrm{H}(M, \emptyset, \omega) .
$$

In addition, a special property for $\mathfrak{s l}(2 \mid 1)$ is that $\delta=1$ (see [17, Lemma $4.13])$.

8.2. Comparison with invariants from nilpotent weight modules of unrolled quantum group. In [6] an invariant of a compatible triple $(M, \Gamma, \omega)$ where $\Gamma$ is a blue $\mathscr{C}^{H}$-ribbon graph embedded in $M$ was defined from the data of a relative premodular ${ }^{5} G$-category $\mathscr{C}^{H}$ with translation group $Z$. Many examples of unrolled quantum groups lead to such categories and in particular quantum groups of Lie algebras at suitable root of unity. Let us discuss how relative premodular categories fit into this paper.

Let $\Lambda$ be a lattice and $W$ a $\Lambda$-graded vector space which give rise to an algebra $\mathcal{U}$ satisfying Axioms 1 and 2. One of the requirements of

\footnotetext{
${ }^{5}$ In [6] the term relative modular is used instead of relative premodular, later in [8] it was shown that an additional requirement is need to define a TQFT and so the use of term was changed.
} 
a relative premodular category is the existence of a translation group. The lattice naturally gives a translation group, in the category of weight modules, which is the family of one dimensional modules $\left(\mathbb{C}_{\lambda}\right)_{\lambda \in \Lambda}$ where $w \in W$ acts by multiplication by $\varepsilon(w)$ and $H_{i}$ by $\ell \lambda\left(H_{i}\right)$. Here our group $G$ is $\mathfrak{H}^{*} / \Lambda$. Another requirement of relative premodular $G$ category is the (generic) semisimplicity of the $\mathscr{C}_{g}^{H}$ for $g \in G \backslash X$ with $X$ being "small." For the rest of the subsection, we assume that $\mathcal{U}$ satisfies Axioms $1-5$ and that $\mathscr{C}^{H}$ is a relative premodular $G$-category. We have proved this is the case for all semi-restricted quantum groups associated to simple Lie algebras.

Let $\bar{\alpha} \in G \backslash X$ so that $\mathcal{U}_{\bar{\alpha}}$ is semisimple. Let $\Theta_{\bar{\alpha}}$ be a set of simple $\mathcal{U}_{\bar{\alpha}}^{H_{-}}$ module such that $\left\{\operatorname{ResF}\left(V_{i}\right): V_{i} \in \Theta_{\bar{\alpha}}\right\}$ is a set of representant of the isomorphism classes of simple $\mathcal{U}_{\bar{\alpha}}$-modules. Then Equation (48) implies that the character given by the Kirby color $x \mapsto \sum_{V_{i} \in \Theta_{\bar{\alpha}}} d_{i} \operatorname{tr}_{\mathbb{C}}\left(\rho_{V_{i}}(x)\right)$ is equal on $\mathcal{U}_{\bar{\alpha}}$ to the symmetrized integral $\mu_{\bar{\alpha}}$. Hence we get

Proposition 8.1. Let $\Gamma$ be a monochrome blue $\mathscr{C}^{H}$-ribbon graph and $(M, \Gamma, \omega)$ a compatible triple. The invariant $\mathrm{N}_{\mathscr{C}^{H}}(M, \Gamma, \omega)$, defined in [6], coming from the relative premodular category $\mathscr{C}^{H}$ is equal to the modified Hennings invariant $\mathrm{H}^{\prime}(M, \Gamma, \omega)$.

Proof. As in [6], choose a computable presentation of $(M, \Gamma, \omega)$ then one gets a diagram where the surgery components are colored by Kirby colors of degree $\bar{\alpha} \notin X$. From Proposition 5.5, the invariant of this diagram is equal to the invariant $F_{\mu}^{\prime}$ of the diagram obtained by replacing the Kirby-colored component with red component. But this is precisely the process for computing $\mathrm{H}^{\prime}$.

8.3. Comparison with the modified Hennings invariants. Let $\mathcal{U}$ be an algebra as in Section 2 satisfying Axioms $1-5$ and $\mathcal{U}^{H}$ its unrolled version. Recall that the quotient $\mathcal{U}_{\overline{0}}^{H}$ of $\mathcal{U}^{H}$ contains a finite dimensional ribbon Hopf algebra $\mathcal{U}_{\overline{0}}$ with the same R-matrix $\mathcal{R}_{\overline{0}} \in \mathcal{Q}_{2} \mathcal{U}^{\otimes 2} / I_{(\overline{0}, \overline{0})} \simeq$ $\mathcal{U}_{\overline{0}} \otimes \mathcal{U}_{\overline{0}}$. There is an obvious associated ribbon functor $\mathscr{C}_{\overline{0}}^{H} \rightarrow \mathcal{U}_{\overline{0}}$-Mod. The construction of [10] produces a modified Hennings invariant since Axiom 4 ensure that $\mathcal{U}_{\overline{0}}$ is a non-degenerate finite dimensional Hopf algebra.

Theorem 8.2. Let $(M, \Gamma, \omega)$ be compatible triple with $\omega=0$. Then the invariant $\mathrm{H}^{\prime}(M, \Gamma, \omega)$ of this paper, associated to $\mathcal{U}$, is equal to the (non graded) modified Hennings invariant of [10] associated to the finite dimensional ribbon Hopf algebra $\mathcal{U}_{\overline{0}}$.

Proof. If $\omega=0$, then the definition of $\mathrm{H}^{\prime}$ only uses the integral $\mu_{\overline{0}}=$ $\lambda_{\overline{0}}(g \cdot)$ and the computation of the universal invariant can be done directly in $\mathcal{U}_{\overline{0}}$ as it is done in [10]. 
Remark that combining this theorem with Proposition 8.1 gives a new proof that the invariants of [6] and [10] coincide for zero cohomology classes, as was first shown in [9].

\section{REFERENCES}

[1] N. Andruskiewitsch and C. Schweigert. On unrolled Hopf algebras. Journal of Knot Theory and Its Ramifications, Vol. 27, No. 10, 1850053 (2018), 2018.

[2] J. Barrett and B. Westbury. Spherical categories. Adv. Math., 143:357 - 375, 1999.

[3] A. Beliakova, C. Blanchet, and A. M. Gainutdinov. Modified trace is a symmetrised integral. arXiv:1801.00321, 2017.

[4] H. Cartan. Variétés analytiques complexes et cohomologie. (French) Colloque sur les fonctions de plusieurs variables, tenu à Bruxelles, pages 41-55, 1953.

[5] V. Chari and A. Pressley. A guide to quantum groups. Cambrige University Press, 1995.

[6] F. Costantino, N. Geer, and B. Patureau-Mirand. Quantum invariants of 3manifolds via link surgery presentations and non-semi-simple categories. Journal of Topology, pages 1005-1053, 2014.

[7] F. Costantino, N. Geer, and B. Patureau-Mirand. Some remarks on the unrolled quantum group of $\mathfrak{s l}(2)$. J. Pure Appl. Algebra, pages 3238-3262, 2015.

[8] M. De Renzi. Non-semisimple extended topological quantum field theories. To Appear in Mem. Amer. Math. Soc., arXiv:1703.07573.

[9] M. De Renzi, N. Geer, and B. Patureau-Mirand. Non-semisimple quantum invariants and TQFTs from small and unrolled quantum groups. preprint to appear in Algebr. Geom. Topol., arXiv:1812.10685, 2018.

[10] M. De Renzi, N. Geer, and B. Patureau-Mirand. Renormalized Hennings invariants and 2+1-TQFTs. Commun. Math. Phys., 362 (3):855 - 907, 2018.

[11] N. Geer, J. Kujawa, and B. Patureau-Mirand. M-traces in (non-unimodular) pivotal categories. arXiv:1809.00499, 2018.

[12] N. Geer and B. Patureau-Mirand. Topological invariants from unrestricted quantum groups. Algebraic \&3 Geometric Topology, 2013.

[13] N. Geer and B. Patureau-Mirand. The trace on projective representations of quantum groups. Letters in Mathematical Physics, Volume 108, Issue 1:117$140,2018$.

[14] N. Geer, B. Patureau-Mirand, and V. Turaev. Modified quantum dimensions and re-normalized links invariants. Compositio Mathematica, pages 196-212, 2009.

[15] N. Geer, B. Patureau-Mirand, and A. Virelizier. Traces on ideals in pivotals categories. Quantum Topology, 4, No. 1:91-124, 2013.

[16] A. Grothendieck. Résumé des résultats essentiels dans la théorie des produits tensoriels topologiques et des espaces nucléaires. Annales de l'Institut Fourrier, tome 4:p. 73-112, 1952.

[17] N. P. Ha. A Hennings type invariant of 3-manifolds from a topological Hopf superalgebra. arXiv:1806.08277, pages 1-35, 2018.

[18] N. P. Ha. Modified trace from pivotal Hopf G-coalgebras. Journal of Pure and Applied Algebra, 224(5):106225, 2020.

[19] I. Heckenberger. Lusztig isomorphisms for drinfel'd doubles of bosonizations of nichols algebras of diagonal type. Journal of Algebra, 323:2130 - 2182, 2010.

[20] M. Hennings. Invariants of links and 3-manifolds obtained from Hopf algebras. Journal of the London Mathematical Society, 54:594-624, 1996.

[21] C. Kassel. Quantum Groups. Springer-Verlag, 1995. 
[22] L. Kauffman and D. E. Radford. Oriented quantum algebras, categories and invariants of knots and links. Journal of Knot Theory and Its Ramifications, 10(07):1047-1084, 2001.

[23] R. Kirby. A calculus for framed links. Invent. Math., 45:35-56, 1978.

[24] S. Lentner and T. Ohrmann. Factorizable $\mathcal{R}$-Matrices for Small Quantum Groups. SIGMA, 13, Number 076:1-25, 2017.

[25] V. Lyubashenko. Invariants of 3-manifolds and projective representations of mapping class groups via quantum groups at roots of unity. Communications in Mathematical Physics, 172, Issue 3:467-516, September 1995.

[26] T. Ohtsuki. Colored ribbon Hopf algebras and universal invariants of framed links. J. Knot Theory Ramifications 2 (2), pages 211-232, 1993.

[27] T. Ohtsuki. Quantum invariants. World Scientific Publishing Co. Pte. Ltd, 2002.

[28] M. J. Pflauma and M. Schottenloher. Holomorphic deformation of Hopf algebras and applications to quantum groups. Journal of Geometry and Physics, Volume 28, Issues 1-2:31-44, 1998.

[29] D. E. Radford. Hopf Algebras. World Scientific, 2012.

[30] J. Roberts. Kirby calculus in manifolds with boundary. Turkish J. Math., 21:111-117, 1997.

[31] F. Trèves. Topological vector spaces, Distributions and Kernels, volume 25. Academic Press Inc., 1967.

[32] V. Turaev. Crossed group-categories. preprint arXiv math/0005291, 2000.

[33] V. Turaev. Homotopy Quantum Field Theory. European Mathematical Society, 2010.

[34] V. G. Turaev. Quantum invariants of knots and 3-manifolds, volume 18 of De Gruyter Studies in Mathematics. Walter de Gruyter \& Co., Berlin, 1994.

[35] A. Virelizier. Algèbres de Hopf graduées et fibrés plats sur les 3-variétés. Thèse de doctorat, Université Louis Pasteur, 2001.

[36] A. Virelizier. Hopf group-coalgebra. Journal of Pure and Applied Algebra, 171:75-122, 2002.

Utah State University, Department of Mathematics and Statistics, LOGAN UT 84341, USA

E-mail address: nathan.geer@usu.edu

Hung Vuong University, Faculty of Natural Sciences, Viet Tri, Phu Tho, Viet NAM

E-mail address: ngocphu.ha@hvu.edu.vn

Université Bretagne Sud, Laboratoire de Mathématiques de Bretagne Atlantique, UMR CNRS 6205, Campus de Tohannic, BP 573 F56017 Vannes, France

E-mail address: bertrand.patureau@univ-ubs.fr 